\title{
OPERADORES DE COMPOSIÇÃO ENTRE AS ÁLGEBRAS CLÁSSICAS DE FUNÇÕES ANALÍTICAS
}

\author{
Daniela Mariz Silva Vieira \\ DISSERTAÇÃO APRESENTADA \\ $\mathrm{AO}$
}

INSTITUTO DE MATEMÁTICA E ESTATÍSTICA

DA

UNIVERSIDADE DE SÃO PAULO

PARA

OBTENÇÃO DO GRAU

$\mathrm{DE}$

MESTRE EM MATEMÁTICA

\author{
Área de Concentração: Análise \\ Orientadora: Profa. Dra. Mary Lilian Lourenço
}

Durante a elaboração deste trabalho a autora recebeu apoio financeiro do CNPq

-São Paulo, 31 de julho de 2000- 


\title{
Operadores de composição entre as álgebras clássicas \\ de funções analíticas
}

\author{
Este exemplar corresponde à redação final \\ da dissertação devidamente corrigida e \\ defendida por Daniela Mariz Silva Vieira \\ e aprovada pela comissão julgadora.
}

São Paulo, 29 de Maio de 2000.

Banca examinadora:

- Profa. Dra. Mary Lilian Lourenço (Orientadora) - IME - USP

- Prof. Dr. Alfredo Jorge Aragona Vallejo - IME - USP

- Prof. Dr. Jorge Tulio Mujica Ascui - IMECC - UNICAMP 
à minha mãe Ana Maria, ao meu pai José e ao meu irmão Felipe. 


\section{O país dos sonhos}

Era um imenso acampamento ao ar livre.

Das cartolas dos magos brotavam alfaces cantoras e pimentões luminosos, e por todas as partes havia gente oferecendo sonhos para trocar. Havia os que queriam trocar um sonho de viagem por um sonho de amores, e havia quem oferecesse um sonho para rir a troco de um sonho para chorar um pranto gostoso.

Um senhor andava ao léu buscando os pedacinhos de seu sonho, despedaçado por culpa de alguém que o tinha atropelado: o senhor ia recolhendo os pedacinhos e os colava e com eles fazia um estandarte cheio de cores.

O aguadeiro de sonhos levava água aos que sentiam sede enquanto dormiam. Levava a água nas costas, em uma jarra, e a oferecia em taças altas.

Sobre uma torre havia uma mulher, de túnica branca, penteando a cabeleira, que chegava aos seus pés. O pente soltava sonhos, com todos seus personagens: os sonhos saíam dos cabelos e iam embora pelo ar.

Eduardo Galeano 


\section{Agradecimentos}

Quero agradecer a todos que realmente acreditaram em mim, durante estes anos de mestrado. A força e confiança que recebi destas pessoas foi essencial para a execução deste trabalho. Sem este apoio, posso afirmar com toda certeza, eu não teria chegado ao fim desta etapa.

Agradeço sobretudo à minha orientadora e amiga Lilian, por ter acreditado em mim e me convidado para estudar com ela, quando eu ainda era aluna de graduação. Agradeço pela orientação, paciência e companheirismo durante estes anos. Impossível descrever aqui o quanto aprendi enquanto estive sob sua orientação, o quanto cresci como estudante de matemática. A escolha do tema de estudo foi excelente, impressionou-me a sua visão do trabalho que seguiríamos, desde o meu ingresso no programa de mestrado. A ela meus sinceros agradecimentos.

Agradeço aos meus colegas de mestrado, meus colegas de classe, cuja companhia me trouxe muitas alegrias. Nunca vou esquecer os grupos de estudo, seminários, as inúmeras listas de exercícios que resolvemos e tiramos dúvidas entre si. Aprendi muito com eles, tanto em matemática, e principalmente quão grande é a generosidade de um amigo. Ana Tereza, Calixto, Célia, Clézio, David Dias, Edson Iwaki, Leonardo, Lymber, Ronaldo, Walter.

Durante a digitação desta dissertação, tive o apoio de pessoas das quais nunca esquecerei. Elas me deram força para não desistir nunca, principalmente quando as dificuldades eram muitas, e as saídas, poucas. Vibraram comigo a cada objetivo alcançado, a cada capítulo terminado. Clésio, Clézio, Cintia da Silva, Hernán, Lilian, Lymber, Marcelo, Renata, Tessie.

Um parágrafo especial quero dedicar ao meus professores que tive nestes anos, com quem aprendi muito, e que para mim foram exemplo de dedicação ao trabalho. Por terem feito com que eu gostasse cada vez mais de matemática, e sobretudo com que eu aprendesse cada vez mais. Aragona, Flávio, Guzzo, Lilian, Lúcia, Marina, Plínio, Rosa.

Mas nem só de livros e teoremas vive um aluno de mestrado em Matemática. Por isso, àqueles que estiveram ao meu lado nas horas de folga sempre serei grata. Pela companhia 
tão importante nas horas de lazer. Cíntia Herkrath, Clésio, Dailton, Eliete, Ernesto, Érika, Henry, Luciano, Lymber, Marcelo, Paulinho, Pipo, Raquel, Renata, Sérgio, Tessie. E um agradecimento muito especial às amigas e técnicos do IME-Softbol, e aos amigos do IME-Beisebol.

Aos amigos do IME, mas que não eram companheiros de mestrado. Amigo eternos, que para sempre ficarão no meu coração. Com quem dividi tantas alegrias, risadas, e as famosas "vagalizações na lanchonete". Autoexec, Daniel Tausk, Henry, Lymber, Masaishi, Renata, São José, Tessie.

Agradeço à minha família, minha avó principalmente, meus tios, tias, primos e primas, pelo amor e pela fé incondicional que sempre tiveram por mim. Alice, Bibi, Cacá, Carol, Cecília, Celina, Celina, Cida, Fabinho, Fê, Fê, Gui, Guiomar, Hugo, João, Lena, Lourdes, Lú, Luís, Mané, Mazé, Mila, Milena, Nino, Sara, Tati, Tioles, Tru, Vitor.

Agradeço ao CNPq pelo auxílio financeiro.

Daniela 


\title{
Resumo
}

Este trabalho tem como objetivo principal estudar operadores de composição entre as álgebras clássicas $\mathcal{H}^{\infty}(D)$ e $\mathcal{A}(D)$ de funções analíticas sobre o disco aberto unitário D. Apresentamos aqui uma caracterização quando os operadores de composição de $\mathcal{A}(D)$ em $\mathcal{A}(D)$ são compactos ou $w$-compactos. Tal caracterização é uma adaptação da demonstração dada por por R. Aron, P. Galindo e M. Lindström em [1] para álgebras que são generalizaçõess naturais das álgebras clássicas. Estudamos operadores da forma $u C_{\varphi}$ e apresentamos a demonstração dada por H. Kamowitz em [19] que caracteriza a compacidade de operadores desta forma e determina seu espectro. Em seguida estudamos homomorfismos $T: \mathcal{H}^{\infty}(D) \longrightarrow \mathcal{H}^{\infty}(D)$, onde apresentamos a caracterização dada por P. Galindo e M. Lindström em [10] para que tais homomorfismos sejam operadores de composição. Finalmente apresentamos a demonstração dada por R. Aron, P. Galindo e M. Lindström em [1], onde provam que todo homomorfismo de $\mathcal{H}^{\infty}(D)$ em $\mathcal{H}^{\infty}(D)$ $w$-compacto é compacto.

\begin{abstract}
The main purpose of this work is to study composition operators between classic algebras $\mathcal{H}^{\infty}(D)$ and $\mathcal{A}(D)$ of analytic functions on the open unit disc $\mathrm{D}$. We shall present here a characterization for compacity and $w$-compacity of a composition operator from $\mathcal{A}(D)$ on $\mathcal{A}(D)$. Such characterization is an adaptation of the proof given by R. Aron, P. Galindo and M. Lindström in [1] for algebras that are natural generalization of the classic algebras. We shall study operators of the form $u C_{\varphi}$ and present the proof given by H. Kamowitz in [19] which characterizes compact operators of this form and determine their spectra. Next we shall study homomorphisms $T: \mathcal{H}^{\infty}(D) \longrightarrow \mathcal{H}^{\infty}(D)$, and we shall present the characterization given by P. Galindo and M. Lindström in [10] which shows when such homomorphisms are composition operators. Finaly we shall present the proof given by R. Aron, P. Galindo and M. Lindström in [1] which proves that every weakly compact homomorphism from $\mathcal{H}^{\infty}(D)$ on $\mathcal{H}^{\infty}(D)$ is compact.
\end{abstract}




\section{Sumário}

$\begin{array}{ll}\text { Introdução } & 3\end{array}$

$\begin{array}{lll}1 & \text { Preliminares } & 7\end{array}$

1.1 Notação . . . . . . . . . . . . . . . . . . . . . . 7

1.2 Definições e Resultados . . . . . . . . . . . . . . . . . . 8

2 Álgebras de Banach $\quad 25$

2.1 Álgebras de Banach . . . . . . . . . . . . . . . . . 25

2.2 Álgebras Uniformes . . . . . . . . . . . . . . . . . . . 32

2.3 Partes de Gleason . . . . . . . . . . . . . . . . . . . . 35

$\begin{array}{lll}3 & \text { A álgebra } \mathcal{A}(D) & 41\end{array}$

3.1 Partes de Gleason de $\mathcal{M}(\mathcal{A}(D)) \ldots \ldots \ldots \ldots$. . . . . . . . . . 41

3.2 Operadores de Composição de $\mathcal{A}(D)$ em $\mathcal{A}(D) \ldots \ldots$. . . . . . . . . . 44

3.3 Operadores Compactos da Forma $u C_{\varphi} \ldots \ldots \ldots \ldots$

3.4 Espectro do Operador Compacto $u C_{\varphi} \ldots \ldots \ldots \ldots$. . . . . . . . 52

4 A álgebra $\mathcal{H}^{\infty}(D) \quad 61$

4.1 Partes de Gleason de $\mathcal{M}\left(\mathcal{H}^{\infty}(D)\right) \ldots \ldots \ldots \ldots$. . . . . . . . . . . . . . 
4.2 Operadores de Composição de $\mathcal{H}^{\infty}(D)$ em $\mathcal{H}^{\infty}(D) \ldots \ldots$. . . . . . . . 64

4.3 Homomorfismos $w$-Compactos de $\mathcal{H}^{\infty}(D)$ em $\mathcal{H}^{\infty}(D) \ldots \ldots \ldots$. . . . . . . 67

Referências Bibliográficas 


\section{Introdução}

O objetivo deste trabalho é estudar propriedades dos operadores de composição entre as álgebras clássicas $\mathcal{H}^{\infty}(D)$ e $\mathcal{A}(D)$ de funções analíticas sobre o disco aberto unitário $D$ de $\mathbb{C}$.

Os operadores de composição aparecem originalmente no trabalho a respeito de mecânica clássica de B. Koopman [21], em 1931. Trinta anos depois, E. Nordgren [28], J. Ryff [30] e H. Schwartz [33] dedicaram-se ao problema de relacionar as propriedades dos operadores de composição com as propriedades da função símbolo. Tais trabalhos propiciaram um programa contínuo de pesquisa sobre os operadores de composição, nos quais incluem, por exemplo, o estudo do espectro e da compacidade dos mesmos.

Nos últimos anos, o problema de caracterizar operadores de composição compactos ou fracamente compactos sobre certas álgebras de Banach de funções analíticas, que são generalizações naturais das álgebras clássicas de funções analíticas, vem sendo estudado por muitos matemáticos. Como por exemplo, Aron [1], Galindo [1, 10, 11, 12], Lindström [1, 11, 12], Lourenço [11], Moraes [11]. Ryan [12], Ülger [34] e outros. Dentre estes trabalhos, destacam-se [1], [10] e [34], que apresentam seções voltadas para o estudo das álgebras clássicas de funções analíticas.

A seguir descrevemos os assuntos abordados em cada capítulo. Convém salientar que os principais resultados aqui desenvolvidos encontram-se nos artigos [1], [10], [19] e [34].

No capítulo 1 apresentamos algumas definições e resultados relativos a Funções Analíticas e Análise Funcional, que serão utilizados ao longo deste trabalho. Observamos que tais tópicos podem ser encontrados nos textos [6] e [5], respectivamente.

O capítulo 2, entitulado de Álgebras de Banach, apresenta alguns resultados da teoria 
de álgebras de Banach que serão utilizados nos capítulos 3 e 4 . Na primeira seção apresentamos definições e exemplos, bem como alguns dos principais resultados de álgebras de Banach. Em seguida, continuamos com o estudo de álgebras de Banach de funções contínuas. Definimos o que são álgebras uniformes e estudamos as principais propriedades do espectro e da representação de Gelfand de tais álgebras. Na sequência mostramos que se $A$ é uma álgebra uniforme, então a representação de Gelfand de $A$ também é uma álgebra uniforme. Introduzimos as partes de Gleason de elementos do espectro de uma álgebra uniforme. Particionar o espectro de uma álgebra uniforme em partes de Gleason será útil no estudo de certos homomorfismos no capítulo 4. Os tópicos apresentados sobre álgebras de Banach podem ser encontrados em [26].

O capítulo 3 apresenta a álgebra de disco, denotada por $\mathcal{A}(D)$. Provamos que todo homomorfismo contínuo nesta álgebra é um operador de composição. Isto deve-se ao fato de todo homomorfismo complexo de $\mathcal{A}(D)$ ser uma avaliação, cuja demonstração baseia-se no fato de toda função em $\mathcal{A}(D)$ poder ser aproximada uniformemente por um sequência de polinômios. Em seguida damos uma caracterização para a compacidade e $w$-compacidade de tais operadores. Por fim estudamos os operadores da forma $u C_{\varphi}$, e apresentamos uma caracterização para a compacidade dos mesmos, bem como calculamos seu espectro.

No capítulo 4 estudamos a álgebra de Banach $\mathcal{H}^{\infty}(D)$, que é o conjunto das funções analíticas e limitadas no disco aberto unitário $D$ de $\mathbb{C}$. Na primeira seção estudamos o espectro de $\mathcal{H}^{\infty}(D)$, bem como as partes de Gleason do mesmo. Vários autores estudaram tais partes, como por exemplo I. Schark em [32], A. Kerr-Lawson em [20] e K. Hoffman em [16]. Como tais autores desenvolveram seus estudos sob diferentes pontos de vista, optamos por apresentar os resultados obtidos por K. Hoffman em [16], pois da forma como são apresentados neste trabalho obtemos uma forte ferramenta para provar o principal teorema do capítulo 4. No trabalho [16], K. Hoffman estabelece uma identificação entre as partes de Gleason não triviais do espectro de $\mathcal{H}^{\infty}(D)$ e o disco unitário aberto $D$ de $\mathbb{C}$, através de homeomorfismos. Na seção seguinte, vemos que em $\mathcal{H}^{\infty}(D)$ nem todo homomorfismo contínuo é necessariamente um operador de composição. Neste sentido apresentamos uma condição necessária e suficiente para que um homomorfismo contínuo em $\mathcal{H}^{\infty}(D)$ seja um operador de composição. Em seguida apresentamos uma caracterização para a compacidade e $w$-compacidade de um operador de composição em 
$\mathcal{H}^{\infty}(D)$.

Na última seção deste capítulo apresentamos uma demonstração de que todo homomorfismo $w$-compacto de $\mathcal{H}^{\infty}(D)$ em $\mathcal{H}^{\infty}(D)$ é compacto. Tal resultado foi generalizado em [10] por P. Galindo e M. Lindström. No referido trabalho eles também provam que se $T: \mathcal{H}^{\infty}(D) \longrightarrow B$ é um homomorfismo $w$-compacto e $B$ é uma álgebra uniforme, então $T$ é compacto. Este resultado também foi obtido por A. Ülger, em [34]. A demonstração que apresentamos independe das acima citadas, e foi feita por R. Aron, P. Galindo e M. Lindström em [1]. Em seguida apresentamos um corolário que foi dado em [34], onde prova-se que sob certas hipóteses sobre uma álgebra uniforme $B$, todo homomorfismo $T: \mathcal{H}^{\infty}(D) \longrightarrow B$ é compacto. 
Aula de matemática

(Tom Jobim - Marino Pinto)

Prá que dividir sem raciocinar

Na vida é sempre bom multiplicar

$E$ por $A$ mais $B$ eu quero demonstrar

Que gosto imensamente de você

Por uma fração infinitesimal

Você criou um caso de cálculo integral

E para resolver este problema

Eu tenho um teorema banal

Quando dois meios se encontram

Desaparece a fração

E se achamos a unidade

Está resolvida a questão

Prá finalizar, vamos recordar

Que menos por menos dá mais, amor

Se vão as paralelas ao infinito se encontrar

Por que demoram tanto dois corações a se integrar

Se desesperadamente, incomensuravelmente

Eu estou perdidamente apaixonado

Apaixonado por você 


\section{Capítulo 1}

\section{Preliminares}

\subsection{Notação}

Estaremos utilizando a seguinte notação:

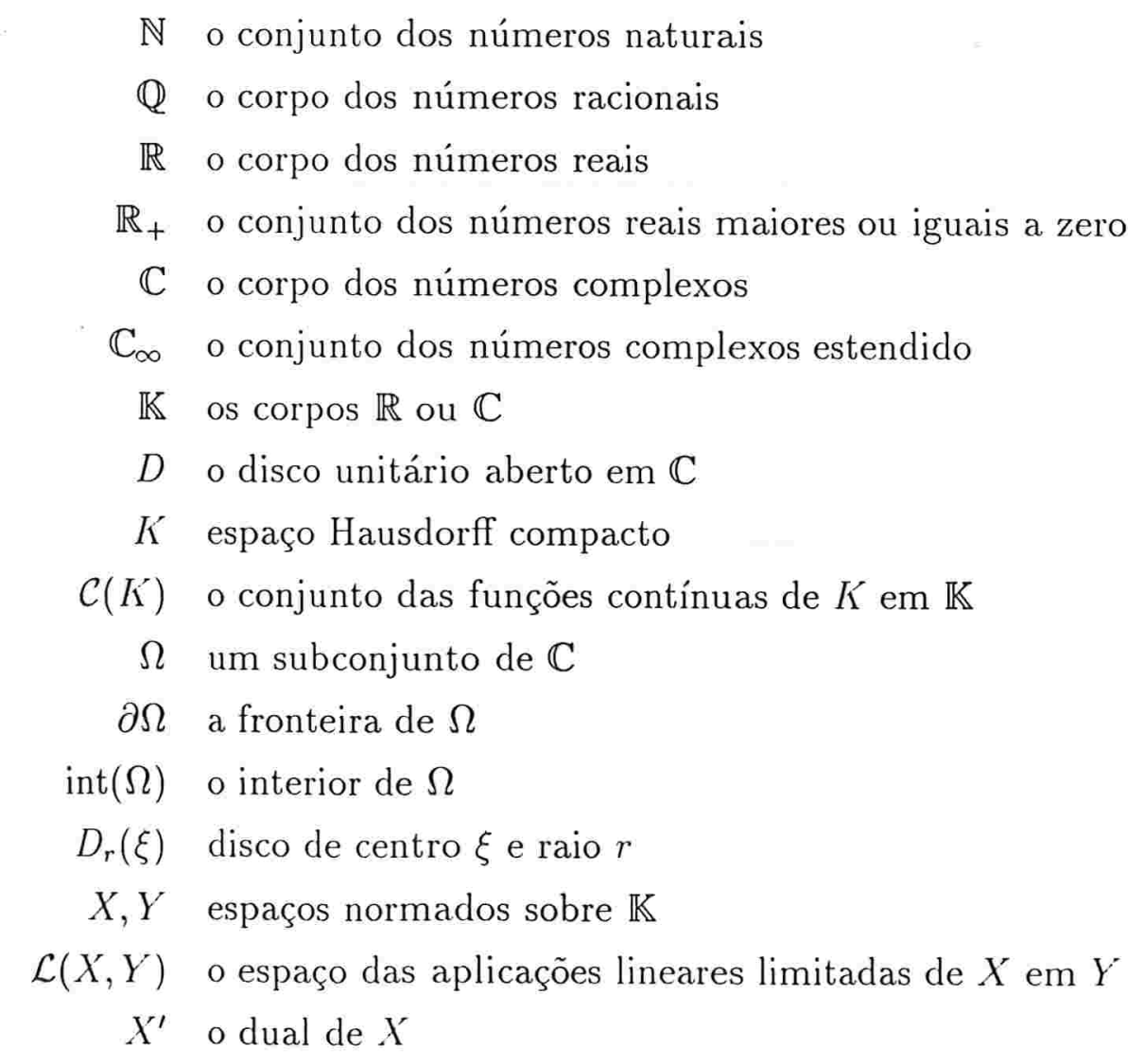




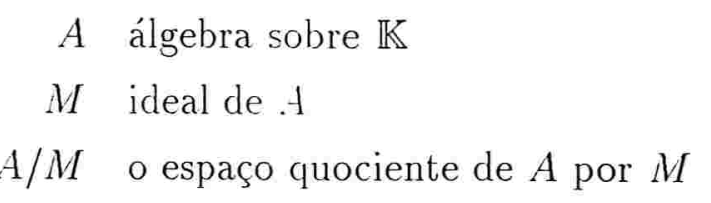

\subsection{Definições e Resultados}

No que se segue estaremos apresentando definições e resultados de Funções Analíticas e Análise Funcional.

\section{Tópicos em Funções Analíticas}

Seja $\Omega$ um aberto não vazio de $\mathbb{C}$. Denotaremos por $\mathcal{H}(\Omega)$ o espaço vetorial de todas as funções analíticas $f: \Omega \longrightarrow \mathbb{C}$. Se $\Omega$ é limitado, chamamos de $\mathcal{A}(\Omega)$ o espaço $\mathcal{H}(\Omega) \cap \mathcal{C}(\bar{\Omega})$, que quando munido da norma dada por

$$
\|f\|=\sup _{z \in \bar{\Omega}}|f(z)|, \forall f \in \mathcal{A}(\Omega)
$$

é um espaço de Banach.

No que se segue, $\Omega$ indicará um subconjunto não vazio de $\mathbb{C}$.

Teorema 1.2.1 Sejam $\Omega$ um aberto de $\mathbb{C}$ ef $f \in \mathcal{H}(\Omega)$. Então $\forall \xi \in \Omega$, existe $r>0$ tal que $D_{r}(\xi) \subseteq \Omega, e$

$$
f(z)=\sum_{m \geq 0} \frac{f^{(m)}(\xi)}{m !}(z-\xi)^{m}, \forall z \in D_{r}(\xi) .
$$

Demonstração: Sugerimos [6], pg. 72 .

Teorema 1.2.2 Sejam $\Omega$ um aberto conexo de $\mathbb{C}$ e $f \in \mathcal{H}(\Omega)$. São equivalentes:

1. existe $\xi \in \Omega$ tal que $f^{(m)}(\xi)=0, \forall m \in \mathbb{N}$;

2. existem $\xi \in \Omega$ er $>0$ tais que $D_{r}(\xi) \subseteq \Omega$ e $f_{\mid D_{r}(\xi)}=0$;

3. existe um aberto não vazio $W$ de $\Omega$ tal que $f_{\mid W}=0$ : 


$$
\text { 4. } f=0 \text {. }
$$

Demonstração: (1) $\Rightarrow(2)$ Vamos supor que $\exists \xi \in \Omega$ tal que $f^{(m)}(\xi)=0, \forall m \in \mathbb{N}$. Pelo Teorema 1.2.1, temos que $\exists r>0$ tal que $D_{r}(\xi) \subseteq \Omega$ e $f(z)=\sum_{m \geq 0} \frac{f^{(m)}(\xi)}{m !}(z-\xi)^{m}, \forall z \in$ $D_{r}(\xi)$, isto é, $f_{\mid D_{r}(\xi)}=0$.

(2) $\Rightarrow$ (4) Consideremos os seguintes conjuntos:

$$
\Omega_{1}=\left\{z \in \Omega: \exists \rho>0 \text { tal que } D_{\rho}(z) \subseteq \Omega \text { e } f_{\left.\right|_{D_{\rho}(z)}}=0\right\} \text { e } \Omega_{2}=\Omega \backslash \Omega_{1} .
$$

É claro que $\Omega=\Omega_{1} \cup \Omega_{2}$ e $\Omega_{1} \cap \Omega_{2}=\emptyset$. $\xi \in \Omega_{1}$.

Não é difícil ver que $\Omega_{1}$ é aberto e que $f_{\left.\right|_{\Omega_{1}}}=0$. Além disso, $\Omega_{1} \neq \emptyset$, pois por hipótese,

Se mostrarmos que $\Omega_{2}$ é aberto, como $\Omega$ é conexo, teríamos que $\Omega_{2}$ é vazio, já que $\Omega_{1} \neq \emptyset$. Por consequência teríamos $\Omega=\Omega_{1}$, o que provaria (4).

Vamos mostrar que $\Omega_{2}$ é aberto, para o que basta verificar que se $z_{0} \in \Omega_{2}$, existe $s>0$ tal que $D_{s}\left(z_{0}\right) \subseteq \Omega_{2}$, ou equivalentemente, que $\exists s>0$ com $D_{s}\left(z_{0}\right) \cap \Omega_{1}=\emptyset$. Vamos supor por absurdo que $D_{s}\left(z_{0}\right) \cap \Omega_{1} \neq \emptyset, \forall s>0$.

Fixemos $\delta>0$ tal que $V=D_{\delta}\left(z_{0}\right) \cap \Omega_{1} \neq \emptyset$.

Como $f_{\left.\right|_{\Omega_{1}}}=0$, temos que $f_{\left.\right|_{V}}=0$ e portanto $f_{\left.\right|_{V}}^{(m)}=0, \forall m \in \mathbb{N}$.

Vamos mostrar agora que $f^{(m)}\left(z_{0}\right)=0, \forall m$. Novamente vamos supor por absurdo que existe $m \in \mathbb{N}$ tal que $f^{(m)}\left(z_{0}\right) \neq 0$. Logo existe $\sigma>0$ tal que $0 \notin D_{\sigma}\left(f^{(m)}\left(z_{0}\right)\right)$, e como $f^{(m)}$ é contínua, existe $s \in[0, \delta]$ tal que $f^{(m)}\left(D_{s}\left(z_{0}\right)\right) \subseteq D_{\sigma}\left(f^{(m)}\left(z_{0}\right)\right)$.

Agora como $D_{s}\left(z_{0}\right) \cap \Omega_{1} \subseteq V$ temos que $f_{\left.\right|_{D_{s}\left(z_{0}\right) \cap \Omega_{1}} ^{(m)}}^{(m)}=0$, e consequentemente

$$
0 \in f^{(m)}\left(D_{s}\left(z_{0}\right) \cap \Omega_{1}\right) \subseteq f^{(m)}\left(D_{s}\left(z_{0}\right)\right) \subseteq D_{\sigma}\left(f^{(m)}\left(z_{0}\right)\right)
$$

o que é um absurdo. Logo $f^{(m)}\left(z_{0}\right)=0, \forall m$, e portanto, pelo Teorema 1.2.1, existe $\epsilon>0$ tal que $D_{\epsilon}\left(z_{0}\right) \subseteq \Omega$ e $f_{\left.\right|_{D_{\epsilon}\left(z_{0}\right)}}=0$, o que implica $z_{0} \in \Omega_{1} \cap \Omega_{2}$, o que é uma contradição. Logo $\Omega_{2}$ é aberto, como queríamos demonstrar.

Finalmente, é claro que (4) $\Rightarrow(1)$ e que (2) e (3) são equivalentes, concluindo a demonstração do teorema. 
Corolário 1.2.3 (Princípio do Prolongamento Analítico) Sejam $\Omega$ um aberto conexo de $\mathbb{C}$ e $f, g \in \mathcal{H}(\Omega)$. Se existir um aberto não vazio $W \subseteq \Omega$ tal que $f_{\left.\right|_{W}}=g_{\left.\right|_{W}}$ então $f=g$.

Demonstração: Considere $h=f-g$. Então temos que $h_{\mid W}=0$. Por 1.2.2, concluímos que $h=0$ em $\Omega$, ou seja, $f=g$ em $\Omega$.

Proposição 1.2.4 Sejam $\Omega$ um aberto conexo de $\mathbb{C}$ e $f \in \mathcal{H}(\Omega)$ tal que $f \neq 0$. Suponha que existe $\xi \in \Omega$ tal que $f(\xi)=0$. Então existem $n \in \mathbb{N}$ e $g \in \mathcal{H}(\Omega)$ tais que $g(\xi) \neq 0$ e

$$
f(z)=(z-\xi)^{n} g(z), \forall z \in \Omega \text {. }
$$

Demonstração: Sugerimos [6], pg. 79.

Teorema 1.2.5 (Princípio dos Zeros Isolados) Sejam $\Omega$ um aberto conexo de $\mathbb{C}, f \in$ $\mathcal{H}(\Omega)$ não constante e $\xi \in \Omega$ tal que $f(\xi)=0$. Então $\exists r>0$ tal que $D_{r}(\xi) \subseteq \Omega$ e $f(z) \neq 0$, $\forall z \in D_{r}(\xi) \backslash\{\xi\}$.

Demonstração: Sugerimos [6], pg. 79 .

Corolário 1.2.6 Se $\Omega$ é um aberto conexo de $\mathbb{C}$ então $\mathcal{H}(\Omega)$ é um anel de integridade.

Demonstração: Sejam $f, g \in \mathcal{H}(\Omega)$ tais que $f \cdot g=0$. Queremos provar que $f=0$ ou $g=0$. Vamos supor por absurdo que $f$ e $g$ não são identicamente nulas. Então existe $\xi \in \Omega$ tal que $f(\xi) \neq 0$. Para tal $\xi$ temos que $g(\xi)=0$.

Como $g$ não é constante segue do Teorema 1.2.5, Princípio dos Zeros Isolados, que existe $r>0$ tal que $D_{r}(\xi) \subseteq \Omega$ e $g(z) \neq 0, \forall z \in D_{r}(\xi) \backslash\{\xi\}$. Logo $f(z)=0, \forall z \in$ $D_{r}(\xi) \backslash\{\xi\}$.

Agora, pelo Teorema 1.2.2 temos que $f=0$, uma contradição. Logo segue o resultado.

Teorema 1.2.7 (Teorema da Aplicação Aberta) Sejam $\Omega$ um aberto conexo de $\mathbb{C} e$ $f \in \mathcal{H}(\Omega), f$ não constante. Se $U$ é um aberto de $\Omega$, então $f(U)$ é aberto. 
Demonstração: Sugerimos [6], pg. 99.

Teorema 1.2.8 (Teorema da Função Inversa) Sejam $\Omega$ um aberto conexo de $\mathbb{C}, f \in$ $\mathcal{H}(\Omega)$. Se $f$ é injetora, então:

1. $f: \Omega \longrightarrow f(\Omega)$ é invertivel;

2. $f^{-1}: f(\Omega) \longrightarrow \mathbb{C}$ é analítica;

3. $\left(f^{-1}\right)^{\prime}(w)=\frac{1}{f^{\prime}\left(f^{-1}(w)\right)}, \forall w \in f(\Omega)$.

Demonstração: Sugerimos [6], pg. 99.

Teorema 1.2.9 Sejam $\Omega \subseteq \mathbb{C}$ um aberto conexo, $f \in \mathcal{H}(\Omega)$ e $\xi \in \Omega$ tal que $|f(\xi)| \geq$ $|f(z)|, \forall z \in \Omega$. Então $f$ é constante.

Demonstração: Sugerimos [6], pg. 128.

Teorema 1.2.10 (Princípio do Módulo Máximo) Sejam $\Omega$ um aberto limitado de $\mathbb{C}$ e $f \in \mathcal{A}(\Omega)$. Então o máximo de $|f|$ em $\bar{\Omega}$ é atingido em $\partial \Omega$.

Demonstração: Sugerimos [6], pg. 12S.

Observação 1.2.11 De acordo com o Princípio do Módulo Máximo, se $f \in \mathcal{A}(\Omega)$, temos que

$$
\|f\|=\sup _{z \in \partial \Omega}|f(z)|
$$

Definição 1.2.12 (Transformação de Möbius) Sejam $a, b, c, d \in \mathbb{C}$ tais que

$$
\Delta=\left|\begin{array}{ll}
a & b \\
c & d
\end{array}\right|=a d-b c \neq 0 .
$$

A aplicação $L(z)=\frac{a z+b}{c z+d}$ é denominada Transformação de Möbius.

Considerando $L_{\left.\right|_{\mathbb{a}\left\{\frac{d}{c}\right\}}}: \mathbb{C} \backslash\left\{-\frac{d}{c}\right\} \longrightarrow \mathbb{C} \backslash\left\{\frac{a}{c}\right\}$, temos que $L$ é uma bijeção analítica, cuja inversa também é analítica. 
Teorema 1.2.13 (Lema de Schwarz) Seja $f \in \mathcal{H}(D)$ tal que $|f(z)| \leq 1, \forall z \in D$, e $f(0)=0$. Então:

1. $|f(z)| \leq|z|, \forall z \in D$;

2. $\left|f^{\prime}(0)\right| \leq 1$.

Além disso, se $\left|f^{\prime}(0)\right|=1$ ou se existe $z_{0} \neq 0, z_{0} \in D$ tal que $\left|f\left(z_{0}\right)\right|=\left|z_{0}\right|$, então existe uma constante $\lambda \in \mathbb{C},|\lambda|=1$, tal que $f(z)=\lambda z, \forall z \in D$.

Demonstração: Sugerimos [6], pg. 130.

No que se segue iremos procurar pelas bijeções analíticas que levam $D$ em $D$.

Seja $\alpha \in D$. Chamamos $\varphi_{\alpha}$ a transformação de Möbius dada por:

$$
\hat{\uparrow}_{\alpha}(z)=\frac{z-\alpha}{1-\bar{\alpha} z}
$$

definida em $\left\{z \in \mathbb{C}: z \neq \frac{1}{\bar{\alpha}}\right\}$.

Não é difícil ver que $\varphi_{\alpha} \in \mathcal{A}(D), \forall \alpha \in D$. A seguinte proposição apresenta mais propriedades das aplicações $\varphi_{\alpha}$.

Proposição 1.2.14 Seja $\alpha \in D$. Então

1. $\varphi_{\alpha}$ é uma aplicação bijetora de $D$ sobre $D$;

2. $\varphi_{\alpha}^{-1}=\varphi_{-\alpha}: D \longrightarrow D$;

3. valem as identidades $\varphi_{\alpha}(\partial D)=\partial D, \varphi_{\alpha}^{\prime}(0)=1-|\alpha|^{2}$ e $\varphi_{\alpha}^{\prime}(\alpha)=\frac{1}{1-|\alpha|^{2}}$.

Demonstração: Sugerimos [6], pg. 131.

A proposição acima nos mostra que as aplicações da forma $\varphi_{\alpha}$, para $\alpha \in D$, formam uma parte das bijeções analíticas de $D$ sobre $D$. A próxima proposição mostrará que toda bijeção de $D$ em $D$ é da forma $\lambda \varphi_{\alpha}$, onde $|\lambda|=1$ e $\alpha \in D$.

Teorema 1.2.15 Seja $f: D \longrightarrow D$ uma função analítica bijetora $e \alpha \in D$ tal que $f(\alpha)=0$. Então existe um número complexo $\lambda$ tal que $|\lambda|=1$ ef $f=\lambda \cdot \varphi_{\alpha}$. 
Demonstração: Sugerimos [6], pg. 132.

No que se segue, toda função analítica da forma $\lambda \cdot \varphi_{\alpha}$, onde $|\lambda|=1$ e $\alpha \in D$, será chamada de automorfismo.

Proposição 1.2.16 Sejam $\alpha, \gamma \in D$ e $\beta \in \partial D$. Então existe um automorfismo $L$ tal que $L(\alpha)=\gamma$ e $L(\beta)=1$.

Demonstração: Vamos construir dois automorfismos $L_{1}$ e $L_{2}$ tais que $L_{1}(\alpha)=0$, $L_{1}(\beta)=1, L_{2}(\gamma)=0$ e $L_{2}(1)=1$. Com efeito, não é difícil ver que

$$
L_{1}(z)=\frac{1-\bar{\alpha} \beta}{\beta-\alpha} \cdot \varphi_{\alpha}(z) \text { e } L_{2}(z)=\frac{1-\bar{\gamma}}{1-\gamma} \cdot \varphi_{\gamma}(z)
$$

satisfazem as exigências acima.

Pela Proposição 1.2.14, temos que $L_{1}: D \longrightarrow D$ e $L_{2}: D \longrightarrow D$ são bijetoras. Logo podemos definir a aplicação $L=L_{2}^{-1} \circ L_{1}: D \longrightarrow D$. Temos que $L$ satisfaz

$$
\begin{aligned}
& L(\alpha)=L_{2}^{-1}\left(L_{1}(\alpha)\right)=L_{2}^{-1}(0)=\gamma \\
& L(\beta)=L_{2}^{-1}\left(L_{1}(\beta)\right)=L_{2}^{-1}(1)=1
\end{aligned}
$$

Como $L_{1}$ e $L_{2}$ são bijeções de $D$ em $D$, temos que $L$ também o é. Portanto, pelo Teorema 1.2.15, temos que $L$ é um automorfismo. Logo $L$ é a aplicação procurada.

Teorema 1.2.17 (Lema de Schwarz-Pick) Seja $f \in \mathcal{A}(D)$ tal que $\|f\| \leq 1$. Então

$$
|f(\alpha)-f(\beta)| \leq\left|\frac{\alpha-\beta}{1-\bar{\beta} \alpha}\right| \cdot|1-\overline{f(\beta)} f(\alpha)|, \forall|\alpha| \leq 1 \text { e }|\beta|<1
$$

Demonstração: Fixemos $\beta \in D$ e $\alpha \in \bar{D}$. Se $|f(\beta)|=1$, segue do Teorema 1.2 .9 que $f$ é constante, e neste caso a desigualdade é facilmente verificada.

Se $|f(\beta)|<1$, tomemos $L$ e $M$ da seguinte maneira:

$$
L(z)=\frac{z+\beta}{1+\bar{\beta} z} \text { e } M(z)=\frac{z-f(\beta)}{1-\overline{f(\beta)} z} .
$$

Desta forma $L, M \in \mathcal{A}(D)$ e se $g=M \circ f \circ L$, então $g \in \mathcal{A}(D),\|g\| \leq 1$ e $g(0)=0$, pois $g(0)=M(f(L(0)))=M(f(\beta))=0$. 
Pelo Teorema 1.2.13 (Lema de Schwarz), temos que $|g(w)| \leq|w|, \forall w \in D$, ou seja

$$
\left|M\left(f\left(\frac{w+\beta}{1+\bar{\beta} w}\right)\right)\right| \leq|w|, \forall w \in D .
$$

Em particular, se $\alpha \in D$ e $w=\frac{\alpha-\beta}{1-\bar{\beta} \alpha}$, então $w \in D$ e $\alpha=\frac{w+\beta}{1+\bar{\beta} w}$. Logo

$$
|M(f(\alpha))| \leq\left|\frac{\alpha-\beta}{1-\bar{\beta} \alpha}\right|
$$

Agora, como

$$
M(f(\alpha))=\frac{f(\alpha)-f(\beta)}{1-\overline{f(\beta)} f(\alpha)},
$$

consequentemente a demonstração segue para $\alpha \in D$.

Se $\alpha \in \partial D$, então $\left|\frac{\alpha-\beta}{1-\bar{\beta} \alpha}\right|=1$, e portanto precisamos provar que

$$
|f(\alpha)-f(\beta)| \leq|1-\overline{f(\beta)} f(\alpha)|
$$

ou equivalentemente, que $|M(f(\alpha))| \leq 1$, o que é imediato, uma vez que $|f(\alpha)| \leq 1$.

No que se segue, iremos introduzir uma métrica em $\mathcal{H}(\Omega)$, e depois apresentaremos alguns resultados sobre convergência neste espaço.

Proposição 1.2.18 Seja $\Omega \subseteq \mathbb{C}$ um aberto. Então existe uma sequência $\left\{K_{n}\right\}$ de subconjuntos compactos de $\Omega$ tais que $\Omega=\bigcup_{n=1}^{\infty} K_{n}$. Mais ainda, os conjuntos $K_{n}$ podem ser escolhidos de maneira a satisfazerem as seguintes condições:

1. $K_{n} \subseteq \operatorname{int}\left(K_{n+1}\right)$;

2. se $K \subset \Omega$ é compacto então $K \subseteq K_{n}$, para algum $n \in \mathbb{N}$.

Demonstração: Sugerimos [6], pg. 142.

Sejam $\Omega \subseteq \mathbb{C}$ um aberto e $\left\{K_{n}\right\}$ a sequência de conjuntos compactos obtidas na Proposição 1.2.18. Sejam $f, g \in \mathcal{H}(\Omega)$. Para cada $n \in \mathbb{N}$ vamos definir $d_{n}(f, g)=$ $\sup _{z \in K_{n}}|f(z)-g(z)| \mathrm{e}$

$$
d(f, g)=\sum_{n=1}^{\infty}\left(\frac{1}{2}\right)^{n} \frac{d_{n}(f, g)}{1+d_{n}(f, g)}
$$


Proposição 1.2.19 Sejam $\Omega$ um aberto de $\mathbb{C}$ ed definida em (1.1). Então $(\mathcal{H}(\Omega), d)$ é um espaço métrico.

Demonstração: Sugerimos [6], pg. 143.

A topologia gerada pela métrica $d$ é denominada topologia da convergência uniforme sobre os compactos de $\Omega$, um vez que uma sequência $\left(f_{n}\right) \subseteq \mathcal{H}(\Omega)$ é convergente para $f \in \mathcal{H}(\Omega)$ se e somente se para cada compacto $K \subseteq \Omega$ e $\epsilon>0$ existir $n_{0} \in \mathbb{N}$ tal que $\sup _{z \in K}\left|f_{n}(z)-f(z)\right|<\epsilon, \forall n \geq n_{0}$. Para mais detalhes, sugerimos [6], páginas 144 e 151 .

Definição 1.2.20 Sejam $\Omega$ um aberto em $\mathbb{C}$ e $\mathcal{F} \subseteq(\mathcal{H}(\Omega), d)$. Dizemos que $\mathcal{F}$ é uma família normal se cada sequência de elementos de $\mathcal{F}$ possui uma subsequência que converge uniformemente sobre os compactos de $\Omega$ para uma função $f \in \mathcal{H}(\Omega)$.

Definição 1.2.21 Sejam $\Omega$ um aberto em $\mathbb{C} \in \mathcal{F} \subseteq \mathcal{H}(\Omega)$. Dizemos que $\mathcal{F}$ é localmente limitada se para todo $\xi \in \Omega$ existem constantes $M$ e $r>0$ tais que para toda $f \in \mathcal{F}$ temos

$$
|f(z)| \leq M \text { para todo } z \in D_{r}(\xi) .
$$

Teorema 1.2.22 (Teorema de Montel) Sejam $\Omega$ um aberto em $\mathbb{C}$ e $\mathcal{F} \subseteq(\mathcal{H}(\Omega), d)$. São equivalentes:

1. $\mathcal{F}$ é uma família normal;

2. $\mathcal{F}$ é localmente limitada.

Demonstração: Sugerimos [6], pg. 153.

Proposição 1.2.23 Seja $\Omega$ um aberto de $\mathbb{C}$ homeomorfo a $D$. Se $f \in \mathcal{H}(\Omega)$, então existe uma sequência de polinômios $\left(p_{n}\right)$ tal que $p_{n} \longrightarrow f$, uniformemente sobre os compactos de $\Omega$.

Demonstração: Sugerimos [6], pg. 202. 
Proposição 1.2.24 Seja $f \in \mathcal{A}(D)$. Então existe uma sequência de polinômios $\left(p_{n}\right)$ tal que $\left\|p_{n}-f\right\| \longrightarrow 0$.

Demonstração: É equivalente mostrarmos que para todo $\epsilon>0$ existe um polinômio $p$ tal que $\|p-f\|<\epsilon$.

Para cada $0<r<1$ seja $f_{r}$ a função analítica definida por $f_{r}(z)=f(r z), \forall z \in$ $D_{\frac{1}{r}}(0)$.

Pela continuidade uniforme de $f$ em $\bar{D}$, temos que existe $\delta>0$ tal que se $|r-1|<\delta$ então $\sup _{z \in \bar{D}}|f(r z)-f(z)|<\frac{\epsilon}{2}$, ou seja

$$
\sup _{z \in \bar{D}}\left|f_{r}(z)-f(z)\right|<\frac{\epsilon}{2}, \text { para }|r-1|<\delta
$$

Agora seja $r$ tal que $|r-1|<\delta$. Pela Proposição 1.2.23, dado $\epsilon>0$, existe um polinômio $p_{r}$ tal que

$$
\sup _{z \in \bar{D}}\left|p_{r}(z)-f_{r}(z)\right|<\frac{\epsilon}{2}
$$

uma vez que $\bar{D}$ é um compacto de $D_{\frac{1}{r}}(0)$.

Assim, por (1.2) e (1.3) temos que

$$
\left\|p_{r}-f\right\|=\left\|p_{r}-f_{r}+f_{r}-f\right\| \leq\left\|p_{r}-f_{r}\right\|+\left\|f_{r}-f\right\|<\epsilon,
$$

como queríamos.

Teorema 1.2.25 (Teorema de Cauchy) Sejam $\Omega$ um aberto de $\mathbb{C}, \triangle$ um triângulo fechado contido em $\Omega, p \in \Omega$ e $f \in \mathcal{C}(\Omega) \cap \mathcal{H}(\Omega \backslash\{p\})$. Então:

$$
\int_{\partial \Delta} f=\int_{\partial \Delta} f(z) d z=0
$$

Demonstração: Sugerimos [29], pg. 221.

Teorema 1.2.26 (Teorema de Morera) Sejam $\Omega$ um aberto conexo de $\mathbb{C}$ e $f \in \mathcal{C}(\Omega)$ tal que $\int_{\partial \Delta} f=0$, para qualquer triângulo fechado $\triangle$ contido em $\Omega$. Então $f \in \mathcal{H}(\Omega)$. 
Demonstração: Sugerimos [6], pg. 86 .

Corolário 1.2.27 Sejam $\Omega$ um aberto conexo de $\mathbb{C}, p \in \Omega$ e $f \in \mathcal{C}(\Omega) \cap \mathcal{H}(\Omega \backslash\{p\})$. Então $f \in \mathcal{H}(\Omega)$.

Demonstração: Seja $\triangle$ um triângulo fechado contido em $\Omega$. Pelo Teorema 1.2.25 temos que $\int_{\partial \triangle} f=0$. Agora, do Teorema 1.2.26 (Morera), concluímos que $f \in \mathcal{H}(\Omega)$, como queríamos.

\section{Tópicos em Análise Funcional}

Seja $(X,\|\cdot\|)$ um espaço normado. Denotaremos por $B_{X}$ a bola unitária fechada de $X$, isto é, $B_{X}=\left\{x \in X^{\prime}:\|x\| \leq 1\right\}$.

Considere $X^{\prime}$ o espaço de Banach sobre $\mathbb{K}$ formado de todos os funcionais lineares limitados em $X$, munido da norma

$$
\|f\|=\sup _{\|x\| \leq 1}|f(x)| .
$$

Para cada $a \in X, f_{1}, f_{2}, \cdots, f_{n} \in X^{\prime}$ e $\epsilon>0$, definimos o seguinte subconjunto de $X$

$$
U\left(a, f_{1}, f_{2}, \cdots, f_{n}, \epsilon\right)=\left\{x \in X: \sup _{1 \leq k \leq n}\left|f_{k}(x)-f_{k}(a)\right|<\epsilon\right\} .
$$

Definição 1.2.28 (Topologia fraca) Seja $G \subseteq X$ um subconjunto não vazio. Dizemos que $G$ é fracamente aberto ou w-aberto se $\forall a \in G, \exists f_{1}, f_{2}, \cdots, f_{n} \in X^{\prime}$ e $\epsilon>0$ tais que

$$
U\left(a, f_{1}, f_{2}, \cdots, f_{n}, \epsilon\right) \subseteq G
$$

É fácil ver que o conjunto de todos os subconjuntos de $X$ que são fracamente abertos é uma topologia para $X$. A notação $(X, w)$ indicará que estaremos considerando em $X$ a topologia fraca e a chamaremos de topologia fraca em $X$ determinada por $X^{\prime}$.

Observações 1.2 .29

1. o espaço topológico $(X, w)$ é um espaço Hausdor.ff; 
2. o operador identidade $I d:(X,\|\cdot\|) \longrightarrow(X, w)$ é contínuo, ou equivalentemente, todo aberto em $(X . w)$ é aberto em $(X,\|\cdot\|)$.

Definição 1.2.30 Sejam $X$ um espaço topológico e A um conjunto parcialmente ordenado por $\leq$, tal que se $\alpha_{1}, \alpha_{2} \in A$ então existe $\alpha_{3} \in A$ tal que $\alpha_{3} \geq \alpha_{1}$ e $\alpha_{3} \geq \alpha_{2}$. Uma rede em $X$ é um par $(A, x)$ onde $x$ é uma função de $A$ em $X$.

Se $\alpha \in A$, escreveremos $x_{\alpha}$ ao invés de $x(\alpha)$, e usaremos $\left(x_{\alpha}\right)_{\alpha \in A}$ ao invés de $(A, x)$.

Definição 1.2.31 (Convergência fraca) Seja $X$ um espaço normado. Uma rede $\left(x_{\alpha}\right) \subseteq$ $X$ é dita fracamente convergente ou $w$-convergente para $x \in X$ se $\left(x_{\alpha}\right)$ converge para $x$ com respeito à topologia fraca.

Notação: $x_{\alpha} \stackrel{w}{\longrightarrow} x$.

Observação 1.2.32 Não é difícil ver que se $X$ é um espaço normado, então $x_{\alpha} \stackrel{w}{\longrightarrow} x$ se, e somente se, $f\left(x_{\alpha}\right) \longrightarrow f(x), \forall f \in X^{\prime}$. Esta caracterização é uma forte ferramenta quando trabalha-se com sequências w-convergentes.

A seguir daremos um exemplo para ilustrar que convergência fraca não implica convergência na norma.

Exemplo 1.2.33 Seja $1<p<\infty$. Vamos considerar o espaço $l_{p}$, formado por todas as sequências de elementos de $\mathbb{K}$ que são $p$-somáveis, e $q>0$ tal que $\frac{1}{p}+\frac{1}{q}=1$. Sejam

$$
\begin{aligned}
e_{1} & =(1,0,0, \cdots) \\
e_{2} & =(0,1,0, \cdots) \\
\vdots & \\
e_{n} & =(0,0, \cdots, \stackrel{n \text {-ésima }}{1}, 0, \cdots)
\end{aligned}
$$

Então $\left\|e_{n}-e_{m}\right\|=\left(\sum_{i=1}^{\infty}\left|e_{n_{1}}-e_{m_{1}}\right|^{p}\right)^{\frac{1}{p}}=2^{\frac{1}{p}}$, para $n \neq m$. Portanto a sequência $\left(e_{n}\right)$ não é convergente em $l_{p}$. 
No entanto, $\left(e_{n}\right)$ é fracamente convergente para 0 . De fato, se $f \in l_{p}^{\prime}$, então $f(x)=$ $\sum_{k=1}^{\infty} a_{k} x_{k}$, onde $\left(x_{k}\right)=x$ e $\left(a_{k}\right)=a \in l_{q}$. Assim temos que $f\left(e_{n}\right)=a_{n}$. Como $a \in l_{q}$,

temos que $\sum_{n=1}^{\infty}\left|a_{n}\right|^{q}<\infty$, o que mostra que $a_{n} \longrightarrow 0$. Portanto $f\left(e_{n}\right)=a_{n} \longrightarrow 0=$ $f(0)$, se $n \rightarrow \infty$, isto é. $\epsilon_{n} \stackrel{w}{\longrightarrow} 0$, se $n \rightarrow \infty$.

Vamos definir agora, para cada $g \in X^{\prime}, x_{1}, x_{2}, \cdots, x_{n} \in X$ e $\epsilon>0$, o seguinte subconjunto de $X^{\prime}$ :

$$
V\left(g, x_{1}, x_{2}, \cdots, x_{n}, \epsilon\right)=\left\{f \in X^{\prime}: \sup _{1 \leq k \leq n}\left|f\left(x_{k}\right)-g\left(x_{k}\right)\right|<\epsilon\right\}
$$

Definição 1.2.34 (Topologia fraca estrela) Seja $G \subseteq X^{\prime}$ um subconjunto não vazio. Dizemos que $G$ é fraca estrela aberto ou $w^{*}$-aberto se $\forall g \in G$. existem $x_{1}, x_{2}, \cdots, x_{n} \in$ $X$ e $\epsilon>0$ tais que

$$
V\left(g, x_{1}, x_{2}, \cdots, x_{n}, \epsilon\right) \subseteq G
$$

Não é difícil ver que o conjunto de todos os subconjuntos de $X^{\prime}$ que são fraca estrela abertos é uma topologia para $X^{\prime}$. A notação $\left(X^{\prime}, w^{*}\right)$ indicará que estaremos considerando em $X^{\prime}$ a topologia fraca estrela e a chamaremos de topologia fraca estrela em $X^{\prime}$ determinada por $X$.

\section{Observações 1.2 .35}

1. o espaço topológico $\left(X^{\prime}, w^{*}\right)$ é um espaço Hausdorff;

2. o operador identidade Id $:\left(X^{\prime}, w\right) \longrightarrow\left(X^{\prime}, w^{*}\right)$ é contínuo, ou equivalentemente, todo aberto em $\left(X^{\prime}, w^{*}\right)$ é aberto em $\left(X^{\prime}, w\right)$.

Definição 1.2.36 (Convergência fraca estrela) Seja $X$ um espaço normado. Uma rede $\left(f_{\alpha}\right)_{a \in A} \subseteq X^{\prime}$ é dita fraca estrela convergente ou $w^{*}$-convergente para $f \in X^{\prime}$ se $\left(f_{\alpha}\right)_{a \in A}$ converge para $f$ com respeito à topologia $w^{*}$.

Notação: $f_{\alpha} \stackrel{w^{*}}{\longrightarrow} f$. 
Observação 1.2.37 É fácil ver que se $X$ é um espaço normado, então $f_{\alpha} \stackrel{w^{*}}{\longrightarrow} f$ se, e somente se, $f_{\alpha}(x) \rightarrow f(x), \forall x \in X$. Tal caracterização será útil quando estivermos trabalhando com sequências $w^{*}$-convergentes.

Definição 1.2.38 Sejam $X, Y$ espaços normados e $T: X \longrightarrow Y$ uma transformação linear. A aplicação $T^{*}: Y^{\prime} \longrightarrow X^{\prime}$ dada por

$$
T^{*}(f)(x)=f(T(x)), \forall f \in Y^{\prime}, x \in X
$$

é chamada de adjunta de $T$.

É fácil ver que a adjunta de $T$ é uma tranformação linear de $Y^{\prime}$ em $X^{\prime}$; e que se $T \in \mathcal{L}(X, Y)$ então $T^{*} \in \mathcal{L}\left(Y^{\prime}, X^{\prime}\right)$.

Teorema 1.2.39 Sejam $X, Y$ espaços normados e $T: X \longrightarrow Y$ uma aplicação linear. Então $T^{*}:\left(Y^{\prime}, w^{*}\right) \longrightarrow\left(X^{\prime}, w^{*}\right)$ é contínua.

Demonstração: Seja $\left(f_{\alpha}\right)_{a \in A}$ uma rede em $Y^{\prime} w^{*}$-convergente para $f \in Y^{\prime}$. Queremos mostrar que $T^{*}\left(f_{\alpha}\right) \stackrel{w^{*}}{\longrightarrow} T^{*}(f)$. Se $x \in X$ então

$$
T^{*}\left(f_{\alpha}\right)(x)=f_{\alpha}(T(x)) \longrightarrow f(T(x))=T^{*}(f)(x) .
$$

Portanto, pela Observação 1.2 .37 , temos que $T^{*}\left(f_{\alpha}\right) \stackrel{w^{*}}{\longrightarrow} T^{*}(f)$, como queríamos.

Teorema 1.2.40 (Eberlein-Šmulian) Um subconjunto de um espaço de Banach é relativamente w-compacto se, e somente se, é sequencialmente w-compacto.

Demonstração: Sugerimos [9], pg. 430.

Teorema 1.2.41 (Teorema de Alaoglu) Se $X$ é um espaço normado, então $B_{X^{\prime}} e^{\prime}$ $w^{\times}$-compacta.

Demonstração: Sugerimos [27], pg. 149. 
Definição 1.2.42 Um espaço topológico $X$ é separável se existe um subconjunto enumerável $Y$ denso em $X$.

Na proposição seguinte, um número complexo $z$ será chamado de complexo racional se $z$ for da forma $a+b i$, onde $a, b \in \mathbb{Q}$.

Proposição 1.2.43 $\mathcal{A}(D)$ é separácel.

Demonstração: Sabemos que o conjunto de todos os polinômios cujos coeficientes são complexos racionais é enumerável e é denso no conjunto dos polinômios com coeficientes em $\mathbb{C}$. Por outro lado, a Proposição 1.2.24 nos diz que o conjunto dos polinômios com coeficientes em $\mathbb{C}$ é denso em $\mathcal{A}(D)$.

Logo, o conjunto dos polinômios com coeficientes complexos racionais é denso em $\mathcal{A}(D)$, resultando que $\mathcal{A}(D)$ é separável.

Definição 1.2.44 Um espaço topológico Hausdorff $X$ é dito metrizável se $X$ satisfazo primeiro axioma da enumerabilidade.

Teorema 1.2.45 Se X é um espaço de Banach, então $B_{X^{\prime}}$ é $w^{*}$-metrizável se, e somente se, $X$ é separável.

Demonstração: Sugerimos [5], pg. 138.

Definição 1.2.46 Se $X, Y$ são espaços normados e $T: X \longrightarrow Y$ é uma transformação linear, dizemos que $T$ é compacto se $\overline{T\left(B_{X}\right)}$ é compacto em $Y$.

Proposição 1.2.47 Sejam $X, Y$ espaços normados. Então

1. todo operador compacto é limitado;

2. se $\operatorname{dim} X=\infty, I d: X \longrightarrow X$ (o operador identidade) nào é compacto.

Demonstração: Sugerimos [22], pg. 406. 
Teorema 1.2.48 Sejam $X, Y$ espaços normados e $T: X \longrightarrow Y$ um operador linear. Se $T$ é limitado e $\operatorname{dim}(\operatorname{im}(T))<\infty$, entào $T$ é compacto.

Demonstração: Sugerimos [22], pg. 407.

Exemplo 1.2.49 Considere $T: l_{2} \longrightarrow l_{2}$ definido por $T\left(\left(x_{j}\right)\right)=\left(\frac{x_{j}}{j}\right), \forall j \in \mathbb{N}$. Vamos mostrar que $T$ é compacto. Para tal, vamos usar o resultado provado em [22] (Teorema 8.1-5, pg. 408), que garante que se um operador $T: X \longrightarrow Y$, onde $X$ é normado e $Y$ é Banach, for limite uniforme de operadores compactos, então $T$ é compacto.

Consideremos a seguinte sequência de operadores:

$$
\begin{aligned}
T_{1}\left(\left(x_{j}\right)\right) & =\left(x_{1}, 0,0, \cdots\right) \\
T_{2}\left(\left(x_{j}\right)\right) & =\left(x_{1}, \frac{x_{2}}{2}, 0,0, \cdots\right) \\
\vdots & \\
T_{n}\left(\left(x_{j}\right)\right) & =\left(x_{1}, \frac{x_{2}}{2}, \cdots, \frac{x_{n}}{n}, 0,0, \cdots\right)
\end{aligned}
$$

Cada $T_{n}$ é linear, limitada e $\operatorname{dim}\left(T_{n}\right)<\infty, \forall n \in \mathbb{N}$. Logo pelo Teorema 1.2 .48 cada $T_{n}$ é compacto. Mais ainda, se $x \in l_{2}, x=\left(x_{j}\right)$, temos que

$$
\left\|\left(T-T_{n}\right)(x)\right\|^{2}=\sum_{j=n+1}^{\infty} \frac{1}{j^{2}}\left|x_{j}\right|^{2} \leq \frac{1}{(n+1)^{2}}\|x\|^{2} .
$$

Logo se $\|x\| \leq 1$ então

$$
\left\|\left(T-T_{n}\right)(x)\right\| \leq \frac{1}{n+1} \longrightarrow 0, n \longrightarrow \infty .
$$

Portanto $T_{n} \longrightarrow T$ uniformemente, o que mostra que $T$ é compacto.

Definição 1.2.50 Seja $B$ um subespaço de um espaço métrico $X$. O conjunto $B$ é totalmente limitado se para todo $\epsilon>0$ pode-se obter uma decomposição $B \subseteq B\left(x_{1}, \epsilon\right) \cup$ $B\left(x_{2}, \epsilon\right) \cup \cdots \cup B\left(x_{n}, \epsilon\right)$.

Definição 1.2.51 Se $K$ é um espaço topológico Hausdorff compacto e $\mathcal{F} \subseteq \mathcal{C}(K)$, dizemos que $\mathcal{F}$ é equicontínuo se para todo $\epsilon>0$ e para todo $x_{0} \in \Lambda$ existe uma vizinhança $U$ de $x_{0}$ tal que $\left|f(x)-f\left(x_{0}\right)\right|<\epsilon$, para todo $x \in U$ e para toda $f \in \mathcal{F}$. 
Teorema 1.2.52 (Teorema de Arzela-Ascoli) Se $K$ é um espaço topológico Hausdorff compacto e $\mathcal{F} \subseteq \mathcal{C}(K)$ então $\mathcal{F}$ é totalmente limitado se e somente se $\mathcal{F}$ é limitado e equicontínuo.

Demonstração: Sugerimos [5], pg. 179.

Proposição 1.2.53 Seja $B$ um subconjunto de um espaço métrico X. Então

1. se $B$ é relativamente compacto, $B$ é totalmente limitado;

2. se $B$ é totalmente limitado e $X$ é completo, $B$ é relativamente compacto.

Demonstração: Sugerimos [22], pg. 412.

Definição 1.2.54 Sejam $X, Y$ espaços de Banach e $T: X \longrightarrow Y$ uma transformação linear limitada. Dizemos que $T$ é fracamente compacto ou $w$-compacto se ${\overline{T\left(B_{X}\right)}}^{w}$ é $w$-compacto em $Y$.

Definição 1.2.55 Sejam $X$ um espaço vetorial e $A \subseteq X$. Dizemos que $A$ é convexo se $\forall x, y \in A$ e $\forall t \in[0,1]$ tivermos $t x+(1-t) y \in A$.

Proposição 1.2.56 Sejam $X$ um espaço normado e $A \subseteq X$ convexo. Então o fecho de A na norma coincide com o fecho de A na topologia fraca.

Demonstração: Sugerimos [8], pg. 11.

Proposição 1.2.57 Se $X, Y$ são espaços de Banach e $T: X \longrightarrow Y$ é um operador compacto, então $T$ é w-compacto.

Demonstração: Queremos mostrar que ${\overline{T\left(B_{X}\right)}}^{w}$ é $w$-compacto em $Y$. Como $T$ é compacto, temos que $\overline{T\left(B_{X}\right)}\|\cdot\|$ é compacto em $Y$. Pela Observação 1.2.29, a aplicação $I d:(Y,\|\cdot\|) \longrightarrow(Y, w)$ é contínua. Logo $\overline{T\left(B_{X}\right)}\|\cdot\|$ é $w$-compacto em $Y$.

Agora, como $T\left(B_{X}\right)$ é convexo, segue da Proposição 1.2 .56 que $\overline{T\left(B_{X}\right)}\left\|^{\|} \cdot\right\|{\overline{T\left(B_{X}\right)}}^{w}$, concluindo a demonstração.

Vamos a seguir dar um exemplo de um operador $w$-compacto que não é compacto. 
Exemplo 1.2.58 Temos que um espaço de Banach $X$ é reflexivo se, e somente se $B_{X}$ é $w$-compacta ([5], Teorema 4.2, pg. 135). Portanto, se $X$ é um espaço de Banach reflexivo de dimensão infinita, o operador identidade $I d: X \longrightarrow X$ é $w$-compacto, porém pela Proposição 1.2 .47 , não é compacto.

Teorema 1.2.59 Sejam $X, Y$ espaços de Banach e $T: X \longrightarrow Y$ uma transformação linear limitada. Então são equivalentes:

1. Té fracamente compacto;

2. $T^{* *}\left(X^{\prime \prime}\right) \subseteq Y$;

3. $T^{*}$ é fracamente compacto.

Demonstração: Sugerimos [5], pg. 189. 


\section{Capítulo 2}

\section{Álgebras de Banach}

\section{1 Álgebras de Banach}

Os resultados deste capítulo encontram-se em textos básicos de álgebras de Banach, tais como [26] e [23].

Definição 2.1.1 Uma álgebra de Banach é um espaço de Banach complexo A que é também uma álgebra, onde a multiplicação e a norma são ligadas pela desigualdade

$$
\|f g\| \leq\|f\| \cdot\|g\|, \forall f, g \in A
$$

Uma álgebra de Banach é comutativa se $f g=g f, \forall f, g \in A$. Uma álgebra de Banach tem unidade se existe um elemento $\mathbf{1} \in A$ tal que $\|\mathbf{1}\|=1$ e $\mathbf{1} f=f \mathbf{1}=f, \forall f \in A$.

Exemplos 2.1.2 Os seguintes espaços vetoriais, dotados do produto pontual e as normas descritas abaixo, são exemplos de álgebras de Banach.

1. Seja $K$ um espaço Hausdorff compacto. Munido da norma do sup, $\mathcal{C}(K)$ é uma álgebra de Banach comutativa com unidade, onde a função $\mathbf{1}(x)=1, \forall x \in K$ é a unidade desta álgebra.

2. O espaço $\mathcal{A}(D) \subseteq \mathcal{C}(\bar{D})$, munido da norma do sup, é uma álgebra de Banach comutativa com unidade. 
3. Seja $\mathcal{H}^{\infty}(D)=\{f: D \longrightarrow \mathbb{C}: f \in \mathcal{H}(D)$ e $f$ é limitada em $D\}$. Munido da norma do sup, $\mathcal{H}^{\infty}(D)$ é uma álgebra de Banach comutativa com unidade.

4. Seja $X$ um espaço de Banach. Considerando em $\mathcal{L}(X, X)$ a operação de composição e a norma $\|T\|=\sup _{x \in B_{X}}\|T(x)\|$, temos que este espaço torna-se uma álgebra de Banach não comutativa com unidade, onde a unidade é o operador identidade, que será denotado por $I d$.

No que se segue, toda álgebra de Banach será comutativa e com unidade.

Definições 2.1.3 Sejam A uma álgebra de Banach e $f \in A$.

1. Dizemos que $f$ é invertível se existe $g \in A$ tal que $f g=1$. O elemento $g$ será chamado de inverso de $f$ e será denotado por $f^{-1}$.

2. Dizemos que $\lambda \in \mathbb{C}$ é valor espectral de $f$ se $f-\lambda \mathbf{1}$ não é invertivel. Denotamos por $\sigma(f)$ o conjunto de todos os valores espectrais de $f$ e chamaremos $\sigma(f)$ de espectro de $f$.

Proposição 2.1.4 Seja A uma álgebra de Banach. Se $f \in A$ é tal que $\|\mathbf{1}-f\|<1$ então $f$ é invertivel.

Demonstração: Como $\|1-f\|<1$ temos que $\sum_{k=1}^{\infty}\|1-f\|^{k}<\infty$, e consequentemente $\sum_{k=1}^{\infty}(1-f)^{k} \in A$, uma vez que $A$ é completo. Afirmamos que $f^{-1}=1+\sum_{k=1}^{\infty}(1-f)^{k}$. De fato, escrevendo $f=1-(1-f)$, temos que

$$
\begin{gathered}
f \cdot\left(1+\sum_{k=1}^{\infty}(1-f)^{k}\right)=f+(1-(1-f)) \cdot\left(\sum_{k=1}^{\infty}(1-f)^{k}\right)= \\
=f+\sum_{k=1}^{\infty}(1-f)^{k}-\sum_{k=1}^{\infty}(1-f)^{k+1}=f+(1-f)=1 .
\end{gathered}
$$

Portanto $f$ é invertível.

Teorema 2.1.5 Seja A uma álgebra de Banach. Então para todo $f \in A, \sigma(f)$ é um subconjunto não vazio e compacto de $\mathbb{C}$. Mais ainda, se $\lambda \in \mathbb{C} e\|f\|<|\lambda|$ então $f-\lambda \mathbf{1}$ é invertivel. 
Demonstração: Sugerimos [5], pg. 200.

Teorema 2.1.6 Sejam A uma álgebra de Banach e $\phi: A \longrightarrow \mathbb{C}$ um homomorfismo não nulo. Então $\phi$ é contínuo e $\|\phi\|=1$.

Demonstração: Como $\phi(\mathbf{1})=\phi(\mathbf{1} \cdot \mathbf{1})=\phi(\mathbf{1})^{2}$, temos que $\phi(\mathbf{1})=1$ ou $\phi(\mathbf{1})=0$. Por hipótese $\phi$ é não nulo. e portanto temos que $\phi(\mathbf{1})=1$.

Provaremos que $\|\phi\| \leq 1$. Vamos supor por absurdo que existe $f \in A$ tal que $\lambda=\phi(f)$ e $|\phi(f)|=|\lambda|>\|f\|$. Pelo Teorema 2.1.5, temos que $f-\lambda \mathbf{1}$ é invertível. Da relação

$$
\phi(f-\lambda \mathbf{1}) \phi\left((f-\lambda \mathbf{1})^{-1}\right)=\phi(\mathbf{1})=1,
$$

obtemos $\phi(f-\lambda 1) \neq 0$, ou seja, $\phi(f) \neq \lambda$, uma contradição.

Portanto $|\phi(f)| \leq\|f\|, \forall f \in A$. Logo $\phi$ é contínuo e $\|\phi\| \leq 1$. Como $\phi(1)=1$, segue que $\|\phi\|=1$, como queríamos.

Teorema 2.1.7 (Teorema de Gelfand-Mazur) Seja A uma álgebra de Banach. Se A é um corpo, então A é isometricamente isomorfo a $\mathbb{C}$.

Demonstração: Para cada $f \in A$ temos pelo Teorema 2.1.5 que $\sigma(f) \neq \emptyset$. Portanto existe $\lambda \in \sigma(f)$ tal que $f-\lambda \mathbf{1}$ não é invertível. Como $A$ é um corpo segue que $f-\lambda \mathbf{1}=0$, ou seja, $f=\lambda \mathbf{1}$.

Portanto para cada $f \in A$ corresponde um número complexo $\lambda$ tal que $f=\lambda \mathbf{1}$. Afirmamos que tal $\lambda$ é único. De fato, se $f=\lambda \mathbf{1}=\mu 1$ então $(\lambda-\mu) \mathbf{1}=0$, isto é, $\lambda=\mu$. Desta forma podemos definir $\Psi: A \longrightarrow \mathbb{C}$ por $\Psi(f)=\lambda, \forall f \in A$. É fácil ver que $\Psi$ é um isomorfismo que preserva a norma, o que conclui a demonstração do teorema.

Definição 2.1.8 Sejam A uma álgebra de Banach e $f \in A$. Chamamos de raio espectral de $f$ ao número dado por

$$
r(f)=\sup \{|\lambda|: \lambda \in \sigma(f)\} .
$$

Proposição 2.1.9 Se A é uma álgebra de Banach e $f \in A$, então $r(f)=\lim _{n \rightarrow \infty}\left\|f^{n}\right\|^{\frac{1}{n}}$. 
Demonstração: Sugerimos [5], pg. 202.

Apresentaremos a seguir algumas propriedades que dizem respeito aos valores espectrais de operadores lineares limitados.

Definição 2.1.10 Sejam $X$ um espaço normado e $T \in \mathcal{L}(X, X)$. Dizemos que $\lambda \in \mathbb{C}$ é um autovalor de $T$ se existir $x \neq 0$ tal que $T(x)=\lambda x$. Denotaremos por $\operatorname{Aut}(T) o$ conjunto de todos os autovalores de $T$.

Proposição 2.1.11 Sejam $X$ um espaço normado e $T \in \mathcal{L}(X, X)$. Se $\operatorname{dim}(\operatorname{im} T)=1$, então $\operatorname{Aut}(T)$ contém no máximo dois elementos.

Demonstração: Seja $y \neq 0$ tal que $\operatorname{im}(T)=[y]$. Então existe um único $\lambda \in \mathbb{K}$ tal que $T(y)=\lambda y . \operatorname{Logo} \lambda \in \operatorname{Aut}(T)$.

Se $\operatorname{ker}(T)=\{0\}$ é imediato que $\operatorname{Aut}(T)=\{\lambda\}$. Se $\operatorname{ker}(T) \neq\{0\}$, temos que existe $x \neq 0$ tal que $T(x)=0$, isto é, $0 \in \operatorname{Aut}(T)$. Se $\lambda \neq 0$ temos que $0, \lambda \in \operatorname{Aut}(T)$.

Vamos agora mostrar que qualquer outro autovalor não nulo de $T$ é necessariamente igual a $\lambda$. Seja $\beta \in \operatorname{Aut}(T)$ tal que $\beta \neq 0$. Então existe $z \neq 0$ tal que $T(z)=\beta z$. Agora como $z \in \operatorname{im}(T)$, existe $\alpha \in \mathbb{K}, \alpha \neq 0$ tal que $z=\alpha y$. Assim

$$
\beta \alpha y=\beta z=T(z)=\alpha T(y)=\alpha \lambda y, \text { ou seja, }(\lambda-\beta) \alpha y=0 .
$$

Como $\alpha y \neq 0$, segue que $\lambda-\beta=0$, ou seja, $\lambda=\beta$, como queríamos.

Portanto $\operatorname{Aut}(T)$ contém no máximo dois elementos.

Proposição 2.1.12 Sejam $X$ um espaço normado, $T, S \in \mathcal{L}(X, X)$ e S invertivel. Então $\sigma\left(S \circ T \circ S^{-1}\right)=\sigma(T)$.

Demonstração: Temos que

$$
\begin{gathered}
\lambda \in \sigma\left(S \circ T \circ S^{-1}\right) \Leftrightarrow \\
S \circ T \circ S^{-1}-\lambda \cdot I d=L \text { não é invertível } \Leftrightarrow \\
S \circ T-\lambda S=L \circ S \text { não é invertível } \Leftrightarrow
\end{gathered}
$$




$$
\begin{gathered}
T-\lambda \cdot I d=S^{-1} \circ L \circ S \text { não é invertível } \Leftrightarrow \\
\lambda \in \sigma(T) .
\end{gathered}
$$

Portanto $\sigma\left(S \circ T \circ S^{-1}\right)=\sigma(T)$.

Proposição 2.1.13 Sejam $X$ um espaço normado e $T \in \mathcal{L}(X, X)$. Então Aut $(T) \subseteq$ $\sigma(T)$.

Demonstração: Sejam $\lambda$ um autovalor de $T$ e $x \in X, x \neq 0$, o seu autovetor correspondente. Então $T(x)-\lambda x=0$, ou seja, $(T-\lambda \cdot I d)(x)=0, \operatorname{com} x \neq 0$.

Logo $T-\lambda \cdot I d$ não é injetora, e portanto não é invertível. Logo $\lambda \in \sigma(T)$, como queríamos.

A seguir veremos um exemplo de um operador que possui um valor espectral que não é um autovalor.

Exemplo 2.1.14 Seja $T: l_{2} \longrightarrow l_{2}$ tal que $T\left(x_{1}, x_{2}, \cdots\right)=\left(0, x_{1}, x_{2}, \cdots\right)$. É claro que $T$ é limitado e $\|T\|=1$.

Temos que $0 \in \sigma(T)$ pois $T$ não é invertível, já que $T$ não é sobrejetor. Mas 0 não é autovalor de $T$, pois se $T(x)=0$ então $x=0$, o que sabemos ser impossível, pois um autovetor é, por definição, não nulo.

Para termos a igualdade entre o conjunto dos valores espectrais e o conjunto dos autovalores de $T$, precisamos de uma hipótese adicional sobre $T$, como mostra o seguinte teorema.

Teorema 2.1.15 Se X é um espaço de normado e $T: X \longrightarrow X$ é um operador compacto, então todo valor espectral não nulo de $T$ é um autovalor de $T$.

Demonstração: Sugerimos [22], pg. 449.

Definição 2.1.16 Seja A uma álgebra. Um ideal próprio $M$ de $A$ é chamado de ideal maximal se para todo ideal $I$ de A tal que $M \subseteq I \subseteq$ A tivermos $M=I$ ou $I=A$. 
Sejam $A$ uma álgebra e $M$ um ideal de $A$. O espaço quociente $A / M$ é uma álgebra, munido das operações usuais de espaço quociente.

Proposição 2.1.17 Sejam A uma álgebra de Banach e $M$ um ideal maximal de A. Então:

1. $M$ é fechado;

2. A/M é isometricamente isomorfo a $\mathbb{C}$.

\section{Demonstração:}

(1) Seja $M \subseteq A$ um ideal maximal. Como $\bar{M}$ é um ideal e $M \subseteq \bar{M} \subseteq A$, segue que $M=\bar{M}$ ou $\bar{M}=A$. Se $\bar{M}=A$, Como $1 \in A$, existe $f \in M$ tal que $\|1-f\|<1$. Pela Proposição 2.1.4, $f$ é invertível, o que contradiz o fato de $M$ ser um ideal próprio. Logo $M=\bar{M}$.

(2) Como $M$ é maximal, $A / M$ é um corpo. Pelo item (1), $M$ é fechado. Logo $A / M$ é uma álgebra de Banach comutativa com a seguinte norma:

$$
\|f+M\|=\inf \{\|f+h\|: h \in M\}
$$

É claro que $1+M$ é unidade de $A / M$. Pelo Teorema 2.1.7 (Gelfand-Mazur), $A / M$ é isometricamente isomorfo a $\mathbb{C}$, como queríamos.

Definição 2.1.18 Seja A uma álgebra de Banach. Chamaremos de espectro de $A o$ conjunto de todos os ideais maximais de $A$, e o denotaremos por $\mathcal{M}(A)$.

Teorema 2.1.19 Seja A uma álgebra de Banach. Então existe uma bijeção do conjunto dos homomorfismos complexos não nulos de $A$ sobre $\mathcal{M}(A)$.

Demonstração: Seja $T: \mathcal{M}(A) \longrightarrow\{\phi: A \longrightarrow \mathbb{C}, \phi$ é um homomorfismo não nulo $\}$ dada por

$$
T(M)=G \circ \pi_{M}, \forall M \in \mathcal{M}(A),
$$

onde $\pi_{M}$ é a projeção canônica de $A$ sobre $A / M$ e $G$ é o isomorfismo entre $A / M$ e $\mathbb{C}$ obtido na Proposição 2.1.17. 
Se $\phi$ é um homomorfismo complexo não nulo de $A, \operatorname{ker}(\phi)$ é um ideal maximal. Agora, para cada $f \in A$ temos $G \circ \pi_{\operatorname{ker}(\phi)}(f)=G(f+\operatorname{ker}(\phi))=\lambda$, onde $\lambda$ é o único número complexo tal que $f-\lambda \mathbf{1} \in \operatorname{ker}(\phi)$.

Portanto $T(\operatorname{ker}(\phi))(f)=\lambda=\phi(f), \forall f \in A$, o que mostra que $T$ é sobrejetora.

Resta provar que $T$ é injetora. Sejam $M_{1}, M_{2} \in \mathcal{M}(A)$ tais que $T\left(M_{1}\right)=T\left(M_{2}\right)$. Assim $G \circ \pi_{M_{1}}(f)=G \circ \pi_{M_{2}}(f)$, para todo $f \in A$.

Agora, se $a \in M_{1}$ então $G \circ \pi_{M_{2}}(a)=G \circ \pi_{M_{1}}(a)=0$. Consequentemente $a \in M_{2}$. Analogamente prova-se que $M_{2} \subseteq M_{1}$. Portanto $T$ é uma bijeção, como queríamos.

A partir de agora estaremos identificando $\mathcal{M}(A)$ com o conjunto dos homomorfismos complexos não nulos de $A$.

Teorema 2.1.20 Seja A uma álgebra de Banach. Então valem as seguintes afirmações:

1. $\mathcal{M}(A)$ é um subconjunto da bola unitária fechada de $A^{\prime}$;

2. $\mathcal{M}(A)$ é $w^{*}$-compacto.

\section{Demonstração:}

(1) Sabemos, pelo Teorema 2.1.6 que se $\phi \in \mathcal{M}(A)$, então $\|\phi\|=1$. Logo $\mathcal{M}(A) \subseteq B_{A^{\prime}}$.

(2) Vamos mostrar que $\mathcal{M}(A)$ é $w^{*}$-fechado em $B_{A^{\prime}}$ que pelo Teorema 1.2.41 (Alaoglu) é $w^{*}$-compacto, e concluir que $\mathcal{M}(A)$ é $w^{*}$-compacto.

Seja $\left(\phi_{\alpha}\right) \subseteq \mathcal{M}(A)$ uma rede $w^{*}$-convergente para $\phi$. Vamos mostrar que $\phi \in \mathcal{M}(A)$. É claro que $\phi$ é um homomorfismo contínuo.

Resta mostrar que $\phi$ é não nulo. Se $\phi$ fosse nulo, teríamos que $\phi_{\alpha} \stackrel{w^{*}}{\rightarrow} 0$, ou seja, $\phi_{\alpha}(f) \longrightarrow 0, \forall f \in A$. Em particular, $1=\phi_{\alpha}(\mathbf{1}) \longrightarrow 0$, o que é uma contradição. Logo $\phi$ é um homomorfismo não nulo, concluindo a demonstração.

O teorema acima mostra que $\mathcal{M}(A) \subseteq B_{A^{\prime}}$. Desta forma a topologia fraca estrela de $A^{\prime}$ induz em $\mathcal{M}(A)$ uma topologia, que é denominada topologia de Gelfand.

Para cada $f \in A$ definimos $\hat{f}: \mathcal{M}(A) \longrightarrow \mathbb{C}$ da seguinte maneira:

$$
\hat{f}(h)=h(f), \forall h \in \mathcal{M}(A) .
$$


Dizemos que $\hat{f}$ é a tranformada de Gelfand de $f$.

O conjunto $\widehat{A}=\{\hat{f}: f \in A\}$ é denominado representação de Gelfand de A e aplicação $\Phi: A \longrightarrow \widehat{A}$ dada por $\Phi(f)=\hat{f}$ é chamada de transformação de Gelfand.

Como estamos considerando em $\mathcal{M}(A)$ a topologia fraca estrela, segue imediatamente da definição acima que $\hat{f}$ é uma aplicação contínua de $\mathcal{M}(A)$ em $\mathbb{C}$. Desta forma $\widehat{A}$ é uma subálgebra de $\mathcal{C}(\mathcal{M}(A))$.

Proposição 2.1.21 Seja A uma álgebra de Banach. A transformação de Gelfand é um homomorfismo contínuo de A sobre $\widehat{A}$.

Demonstração: É fácil verificar que $\Phi$ é um homomorfismo. Agora, para cada $f \in A$ tem-se

$$
\|\Phi(f)\|=\|\hat{f}\|=\sup _{\phi \in \mathcal{M}(A)}|\hat{f}(\phi)|=\sup _{\phi \in \mathcal{M}(A)}|\phi(f)| \leq \sup _{\phi \in \mathcal{M}(A)}\|\phi\| \cdot\|f\|=\|f\|
$$

ou seja, $\|\hat{f}\| \leq\|f\|$, o que prova que a $\Phi$ é contínua.

Observação 2.1.22 Sabemos que $\mathcal{M}(A) \subseteq A^{\prime}$. No entanto, $\mathcal{M}(A)$ não é um subespaço vetorial de $A^{\prime}$, pois não podemos garantir, por exemplo, que um múltiplo por um escalar de um homomorfismo seja um homomorfismo. Portanto, não podemos considerar $\mathcal{M}(A)$ como um espaço normado. No entanto, se definirmos em $\mathcal{M}(A)$ a seguinte distância:

$$
d\left(h_{1}, h_{2}\right)=\left\|h_{1}-h_{2}\right\| \text { para } h_{1}, h_{2} \in \mathcal{M}(A),
$$

teremos que $\mathcal{M}(A)$ torna-se um espaço métrico.

No que se segue, quando nos referirmos à norma em $\mathcal{M}(A)$, estaremos nos referindo à norma de $A^{\prime}$.

\section{2 Álgebras Uniformes}

Definição 2.2.1 Seja A uma álgebra de Banach. Dizemos que A é uma álgebra uniforme se existe um espaço topológico Hausdorff compacto $K_{\text {tal }}$ que $A \subseteq \mathcal{C}\left(K^{\prime}\right)$ e 
1. $A \subseteq \mathcal{C}(K)$ é fechado;

2. A separa pontos de $K$;

3. A contém as funções constantes.

Exemplo 2.2.2 A álgebra de Banach $\mathcal{A}(D) \subseteq \mathcal{C}(\bar{D})$ é uma álgebra uniforme. Com efeito $\mathcal{A}(D)$ é uma subálgebra fechada de $\mathcal{C}(\bar{D})$, pois o limite uniforme de funções analíticas em $D$ e contínuas em $\bar{D}$ é uma função analítca em $D$ e contínua em $\bar{D}$.

Sejam $z_{1}, z_{2} \in \bar{D}$ distintos. Como id (a função identidade) pertence a $\mathcal{A}(D)$, temos que $i d\left(z_{1}\right)=z_{1} \neq z_{2}=i d\left(z_{2}\right)$. Logo $\mathcal{A}(D)$ separa pontos de $\bar{D}$. Agora é claro que $\mathcal{A}(D)$ contém as funções constantes.

Exemplo 2.2.3 Vamos mostrar que $\mathcal{H}^{\infty}(D)$ é uma álgebra uniforme. Para verificar que $\mathcal{H}^{\infty}(D)$ é fechado, separa pontos de $D$ e contém as constantes, procedemos de maneira análoga à feita no Exemplo 2.2.2.

O menos intuitivo, no caso da álgebra $\mathcal{H}^{\infty}(D)$, é encontrar um espaço topológico Hausdorff compacto $K$ tal que $\mathcal{H}^{\infty}(D) \subseteq \mathcal{C}(K)$.

$\mathrm{Na}$ seção anterior, a partir de uma álgebra de Banach $A$, construímos os conjuntos $\mathcal{M}(A)$ e $\widehat{A} \subseteq \mathcal{C}(\mathcal{M}(A))$, onde $\mathcal{M}(A)$ é $w^{*}$-compacto. Provamos que que a transformação de Gelfand é um homomorfismo contínuo de $A$ sobre $\widehat{A}$.

Porém $\Phi$ é injetora quando $A=\mathcal{H}^{\infty}(D)$. Com efeito, se $f_{1}, f_{2} \in \mathcal{H}^{\infty}(D)$ e $\Phi\left(f_{1}\right)=$ $\Phi\left(f_{2}\right)$, temos que $h\left(f_{1}\right)=h\left(f_{2}\right), \forall h \in \mathcal{M}\left(\mathcal{H}^{\infty}(D)\right)$.

Para cada $z \in D$, seja $\phi: \mathcal{H}^{\infty}(D) \longrightarrow \mathbb{C}$ o homomorfismo complexo dado por $\phi(f)=f(z), \forall f \in \mathcal{H}^{\infty}(D)$. Então $\phi\left(f_{1}\right)=\phi\left(f_{2}\right)$, isto é, $f_{1}(z)=f_{2}(z)$. Como $z$ era arbitrário, temos que $f_{1}=f_{2}$. Logo $\Phi$ é injetora.

Desta forma podemos identificar $\left.\mathcal{H}^{\infty}(D) \operatorname{com} \widehat{\mathcal{H}^{\infty}(D)}\right)$ e como $\widehat{\mathcal{H}^{\infty}(D)} \subseteq \mathcal{C}\left(\mathcal{M}\left(\mathcal{H}^{\infty}(D)\right)\right)$, temos que $\mathcal{H}^{\infty}(D)$ é uma álgebra uniforme.

Definição 2.2.4 Seja $A \subseteq \mathcal{C}\left(K^{\prime}\right)$ uma álgebra uniforme. Para cada $x \in K$, a aplicação $\delta_{x}: A \longrightarrow \mathbb{C}$ dada por

$$
\delta_{x}(f)=f(x), \forall f \in A
$$


é chamada de avaliação.

É fácil ver que cada avaliaçào é um homomorfismo nào nulo. Consequentemente pela Proposição 2.1.6, $\delta_{x}$ é contínua, $\forall x \in K$. Logo $\delta_{x} \in \mathcal{M}(A), \forall x \in K$.

Teorema 2.2.5 Sejam $A \subseteq \mathcal{C}(K)$ uma álgebra uniforme e $\delta: K \longrightarrow \mathcal{M}(A)$ dada por $\delta(x)=\delta_{x}, \forall x \in K$. Então $\delta$ é um homeomorfismo sobre $\delta(K)$.

Demonstração: Sejam $x, y \in K$ tais que $\delta_{x}=\delta_{y}$. Então $f(x)=f(y), \forall f \in A$. Vamos supor por absurdo que $x \neq y$. Como $A$ separa pontos, existe $f \in A$ tal que $f(x) \neq f(y)$, uma contradição. Logo $x=y$ e portanto $\delta$ é injetora.

Para provar que $\delta$ é contínua, sejam $x_{0} \in K$ e $U=\left\{\phi \in \mathcal{M}(A): \sup _{1 \leq j \leq n} \mid \phi\left(f_{j}\right)-\right.$ $\left.\delta_{x_{0}}\left(f_{j}\right) \mid<\epsilon\right\}$ uma vizinhança $w^{*}$-aberta de $\delta\left(x_{0}\right)=\delta_{x_{0}}$, onde $f_{1}, \cdots, f_{n} \in A$. Vamos construir um aberto $V \subseteq K \operatorname{com} x_{0} \in V$ tal que $\delta(x) \in U, \forall x \in V$.

Com efeito, como cada $f_{j}$ é contínua em $x_{0}$, existe um aberto $V \subseteq K, x_{0} \in V$ tal que se $x \in V$, então $\left|f_{j}(x)-f_{j}\left(x_{0}\right)\right|<\epsilon, 1 \leq j \leq n$. Ou seja, $\left|\delta_{x}\left(f_{j}\right)-\delta_{x_{0}}\left(f_{j}\right)\right|<\epsilon, 1 \leq j \leq n$, donde segue que $\delta(x) \in U, \forall x \in V$, como queríamos.

Como $K$ é compacto, segue que $\delta$ é um homeọmorfismo sobre $\delta(K)$.

O teorema acima mostra que os conjuntos $K$ e $\delta(K) \subseteq \mathcal{M}(A)$ são homeomorfòs. Em váriaś ocasiões neste texto éstaremos nos referindo a elementos de $K$ ou de $\delta(K) \subseteq \mathcal{M}(A)$ como o mesmo elemento, mas tendo em mente que estaremos nos referindo à imagem do mesmo pela inclusão $\delta$.

O próximo teorema mostra que se $A$ é uma álgebra uniforme, então $\widehat{A} \subseteq \mathcal{C}(\mathcal{M}(A))$ é uma álgebra uniforme e $\|\hat{f}\|=\|f\|, \forall f \in A$.

Teorema 2.2.6 Seja A uma álgebra uniforme. Então

1. a transformação de Gelfand é um isomorfismo isométrico de A sobre $\widehat{A}$;

2. $\widehat{A} \subseteq \mathcal{C}(\mathcal{M}(A))$ é uma álgebra uniforme. 


\section{Demonstração:}

(1) Pela Proposiçào 2.1.21 temos que $\|\hat{f}\| \leq\|f\|$. Por outro lado, como $\left\{\delta_{x}: x \in K\right\} \subseteq$ $\mathcal{M}(A)$, temos que

$$
\sup _{x \in K}\left|\hat{f}\left(\delta_{x}\right)\right| \leq \sup _{h \in \mathcal{M}(A)}|\hat{f}(h)|=\|\hat{f}\|
$$

ou seja,

$$
\|\hat{f}\| \geq \sup _{x \in K}\left|\hat{f}\left(\delta_{x}\right)\right|=\sup _{x \in K}|f(x)|=\|f\| .
$$

Portanto $\|\hat{f}\|=\|f\|$, o que mostra que a transformação de Gelfand é um isomorfismo isométrico.

(2) Pelo item (1) a transformação de Gelfand é uma isomorfismo isométrico e como $A$ é completo, segue que $\widehat{A}=\Psi(A)$ é completo e portanto fechado.

Sejam $h_{1}, h_{2} \in \mathcal{M}(A)$ tais que $h_{1} \neq h_{2}$. Então existe $f \in A$ tal que $h_{1}(f) \neq h_{2}(f)$. Logo $\hat{f}\left(h_{1}\right) \neq \hat{f}\left(h_{2}\right)$. Portanto $\widehat{A}$ separa pontos de $\mathcal{M}(A)$.

Vamos mostrar que $\widehat{A}$ contem as constantes. Se $h \in \mathcal{M}(A)$ então

$$
\hat{\mathbf{1}}(h)=h(\mathbf{1})=1 \text {. }
$$

Logo $\hat{1}=1$ e portanto $\widehat{A}$ contém aș constantes é é uma álgebra uniforme.

\subsection{Partes de Gleason}

Em 1957, A. Gleason em [14] introduziu uma relação de equivalência no espectro $\mathcal{M}(A)$ de uma álgebra uniforme $A$, a qual divide $\mathcal{M}(A)$ em classes de equivalência chamadas de partes de Gleason. A decomposição de $\mathcal{M}(A)$ nestas partes é fundamental na busca por estruturas analíticas no espaço $\mathcal{M}(A)$, no sentido de que os elementos de $\widehat{A}$ agem de certa forma como funções analíticas quando restritas a uma parte de Gleason.

O estudo de partes de Gleason neste texto será útil na seção 3 do capítulo 4 , onde provaremos que todo homomorfismo $T: \mathcal{H}^{\infty}(D) \longrightarrow \mathcal{H}^{\infty}(D)$ w-compacto é compacto.

Sejam $K$ um espaço topológico Hausdorff compacto, $A \subseteq \mathcal{C}(K)$ uma álgebra uniforme e $a, b \in \mathcal{M}(A)$. Definimos $A_{b}=\{f \in A: \hat{f}(b)=0\}$ e

$$
\psi(a, b)=\sup \left\{|\hat{f}(a)|: f \in A_{b} \text { e }\|f\| \leq 1\right\} .
$$


Note que $v(a, b) \leq 1, \forall a, b \in \mathcal{M}(A)$.

Seja $L: \bar{D} \longrightarrow \bar{D}$ um automorfismo. Então existem $\lambda \in \partial D$ e $\alpha \in D$ tais que $L=\lambda \varphi_{\alpha}$. Para cada $f \in A$ tal que $\|f\| \leq 1$, ou seja, $f(K) \subseteq \bar{D}$, consideremos a função $L \circ f$ dada por

$$
(L \circ f)(x)=L(f(x))=\lambda \frac{f(x)-\alpha}{1-\bar{\alpha} f(x)}, \forall x \in K
$$

Lema 2.3.1 Sejam A uma álgebra uniforme, $f \in A$ tal que $\|f\| \leq 1$ e L um automorfismo. Então

1. $L \circ f \in A$;

2. $\widehat{L \circ f}=L \circ \hat{f}$.

\section{Demonstração:}

(1) Seja $p: \bar{D} \longrightarrow \mathbb{C}$ um polinômio dado por $p(z)=a_{n} z^{n}+\cdots+a_{0}, a_{i} \in \mathbb{C}, i=1, \cdots, n$. Consideremos $f \in A$ com $\|f\| \leq 1$. Se definirmos, para cada $x \in K,(p \circ f)(x)=$ $a_{n} f(x)^{n}+\cdots+a_{0}$, temos que $p \circ f \in A$, já que $A$ é uma álgebra.

Como $L \in \mathcal{A}(D)$, a Proposição 1.2 .24 garante que existe uma sequência $\left(p_{n}\right)$ de polinômios tal que $p_{n} \longrightarrow L$, uniformemente em $\bar{D}$. Ou seja,

$$
\sup _{z \in \bar{D}}\left|p_{n}(z)-L(z)\right| \longrightarrow 0, n \rightarrow \infty .
$$

Em particular, se $f \in A$ e $\|f\| \leq 1$ então

$$
\sup _{x \in K}\left|p_{n}(f(x))-L(f(x))\right| \longrightarrow 0, n \rightarrow \infty,
$$

ou seja, $\lim _{n \rightarrow \infty} p_{n} \circ f=L \circ f$.

Pelo observado acima, para cada $n$ temos que $p_{n} \circ f \in A$, e como $A$ é fechado, temos que $L \circ f \in A$, como queríamos.

(2) Para cada $h \in \mathcal{M}(A)$ temos que

$$
\widehat{L \circ f}(h)=h\left(\lambda \frac{f-1 \alpha}{1-\bar{\alpha} f}\right)=\frac{\lambda(h(f)-\alpha)}{1-\bar{\alpha} h(f)}=\frac{\lambda(\hat{f}(h)-\alpha)}{1-\bar{\alpha} \hat{f}(h)}=L \circ \hat{f}(h) .
$$

Portanto $\widehat{L \circ f}=L \circ \hat{f}$. 
Lema 2.3.2 Sejam A uma álgebra uniforme e $a, b \in \mathcal{M}(A)$. Então $\psi(a, b)<1$ se, e somente se, $\|a-b\|<2$.

Demonstração: Vamos supor que $\psi(a, b)<1$. Se $f \in A$ então $g=f-\hat{f}(b) \in A_{b}$, pois $\hat{g}(b)=b(g)=b(f-\hat{f}(b))=b(f)-\hat{f}(b)=0$.

Agora como $\|g\|=\|f-\hat{f}(b)\| \leq\|f\|+\|f\| \cdot\|b\|=2\|f\|$, temos que $\|g\| \leq 2\|f\|$.

$\operatorname{Assim}|\hat{f}(a)-\hat{f}(b)|=|\hat{g}(a)| \leq \psi(a, b) \cdot\|g\| \leq 2 \psi(a, b) \cdot\|f\|$, isto é, $|a(f)-b(f)| \leq$ $2 \psi(a, b) \cdot\|f\|, \forall f \in A$. o que mostra que $\|a-b\| \leq 2 \psi(a, b)$. Como $\psi(a, b)<1$ segue que $\|a-b\|<2$.

Reciprocamente, se $\psi(a, b)=1$, existe $\left(f_{n}\right) \subseteq A_{b}$ tal que $\left\|f_{n}\right\| \leq 1$ e $\left|\widehat{f}_{n}(a)\right| \longrightarrow 1$.

Para cada $z \in \mathbb{C}$ existe $\theta_{n}(z)$ tal que $f_{n}(z)=g_{n}(z) \mathrm{e}^{-i \theta_{n}(z)}$, onde $\left|f_{n}(z)\right|=g_{n}(z)$. Logo, podemos escrever $g_{n}=f_{n} \mathrm{e}^{i \theta_{n}}$.

Substituindo $f_{n}$ por $g_{n}=\frac{\mathrm{e}^{i \theta n} f_{n}}{1+2^{-n}}$, teremos que $g_{n} \in A_{b},\left|\widehat{g_{n}}(a)\right|<1$ e $\widehat{g_{n}}(a) \longrightarrow 1$.

Agora a Proposição 1.2.16 garante que para cada $n \in \mathbb{N}$ existe um automorfismo $L_{n}$ tal que:

$$
L_{n}(1)=1 \text { e } L_{n}(0)=-1+\frac{1}{2^{n}}
$$

Fixemos $n \in \mathbb{N}$. Como $L_{n}$ é contínua e $\widehat{g_{k}}(a) \longrightarrow 1$, existe $k \in \mathbb{N}$ tal que

$$
\left|L_{n}\left(\widehat{g_{k}}(a)\right)-1\right|<\frac{1}{2^{n}} \text {. }
$$

Seja $h_{n}=L_{n} \circ g_{k}$. Pelo Lema 2.3.1 temos que $h_{n} \in A, \forall n \in \mathbb{N}$. É claro que $\left\|h_{n}\right\| \leq 1$. Assim

$$
\begin{gathered}
\left|\widehat{h_{n}}(a)-\widehat{h_{n}}(b)\right|=\left|L_{n} \circ \widehat{g_{k}}(a)-L_{n} \circ \widehat{g_{k}}(b)\right|=\left|L_{n}\left(\widehat{g_{k}}(a)\right)-L_{n}(0)\right|=\left|L_{n}\left(\widehat{g_{k}}(a)\right)+1-\frac{1}{2^{n}}\right|= \\
=\left|L_{n}\left(\widehat{g_{k}}(a)\right)-1+2-\frac{1}{2^{n}}\right| \leq\left|L_{n}\left(\widehat{g_{k}}(a)\right)-1\right|+2+\frac{1}{2^{n}}<\frac{1}{2^{n}}+2+\frac{1}{2^{n}} \longrightarrow 2 .
\end{gathered}
$$

Logo $\left|a\left(h_{n}\right)-b\left(h_{n}\right)\right| \longrightarrow 2$, se $n \rightarrow \infty$, o que mostra que $\|a-b\|=2$, provando a equivalência.

Lema 2.3.3 Sejam A uma álgebra uniforme e $a, b \in \mathcal{M}(A)$. Então $\psi(a, b)<1$ se, $e$ somente se, para toda sequência $\left(f_{n}\right) \subseteq A$ com $\left\|f_{n}\right\| \leq 1$ e $\hat{f}_{n}(a) \longrightarrow 1$ tivermos $\widehat{f}_{n}(b) \longrightarrow 1$. 
Demonstração: Suponha que para toda sequência $\left(f_{n}\right) \subseteq A$ com $\left\|f_{n}\right\| \leq 1$ e $\widehat{f}_{n}(a) \longrightarrow 1$ temos $\widehat{f}_{n}(b) \longrightarrow 1$.

Se $\psi(a, b)=1$ temos que existe uma sequência $\left(f_{n}\right) \subseteq A_{b}$, com $\left\|f_{n}\right\| \leq 1$ tal que $\left|\widehat{f}_{n}(a)\right| \longrightarrow 1$. Substituindo, como no lema anterior, $f_{n}$ por $g_{n}=e^{i \theta n} f_{n}$, teremos que $\widehat{g_{n}}(a) \longrightarrow 1$. Como $f_{n} \in A_{b}$, temos que $g_{n} \in A_{b}$, o que implica $\widehat{g_{n}}(b)=0$, uma contradição.

Reciprocamente, vamos supor por absurdo que existe $\left(f_{n}\right) \subseteq A,\left\|f_{n}\right\| \leq 1, \widehat{f}_{n}(a) \longrightarrow 1$ mas $\left(\widehat{f}_{n}(b)\right)$ não convergindo para 1 . Novamente, substituindo $f_{n}$ por $g_{n}=\frac{\mathrm{e}^{i \theta_{n}} f_{n}}{1+2^{-n}}$, temos que existe uma subsequência $\left(g_{n_{k}}\right)$ de $\left(g_{n}\right)$ com $\widehat{g_{n_{k}}}(b) \longrightarrow r \neq 1$.

Sem perda de generalidade, sejam $\alpha_{n}=\widehat{g_{n}}(a)$ e $\beta_{n}=\widehat{g_{n}}(b)$. Então $\left|\alpha_{n}\right|<1$ e $\left|\beta_{n}\right|<1$.

Agora, se $|r|<1$, para cada $n \in \mathbb{N}$ tomemos, através da Proposição 1.2.16, o automorfismo $L_{n}$ tal que $L_{n}(1)=1$ e $L_{n}(r)=-1+4^{-n}$. Como $L_{n}$ é contínua existe $k$ tal que

$$
\left|L_{n}\left(\alpha_{k}\right)-1\right|<2^{-n} \text { e }\left|L_{n}\left(\beta_{k}\right)+1\right|<2^{-n} .
$$

Pelo Lema 2.3.1 temos que $h_{n}=L_{n} \circ g_{k} \in A$ e além disso $\left\|h_{n}\right\| \leq 1$. Portanto

$$
\begin{gathered}
\left|\widehat{h_{n}}(a)-\widehat{h_{n}}(b)\right|=\left|L_{n}\left(\alpha_{k}\right)-L_{n}\left(\beta_{k}\right)\right|=\left|L_{n}\left(\alpha_{k}\right)-L_{n}\left(\beta_{k}\right)+1-1\right| \leq \\
\leq\left|L_{n}\left(\alpha_{k}\right)+1\right|+\left|L_{n}\left(\beta_{k}\right)+1\right|<\left|L_{n}\left(\alpha_{k}\right)+1\right|+2^{-n}<\left|L_{n}\left(\alpha_{k}\right)+2-1\right|+2^{-n} \leq \\
\leq\left|L_{n}\left(\alpha_{k}\right)-1\right|+2+2^{-n}<2^{-n}+2+2^{-n} \longrightarrow 2,
\end{gathered}
$$

ou seja $\|a-b\|=2$. Pelo Lema 2.3.2, temos que $\psi(a, b)=1$, o que é uma contradição.

Se $|r|=1$, não é difícil construir um automorfismo $L$ tal que $L(1)=1$ e $L(r)=-1$. Então $h_{n}=L \circ g_{n} \in A,\left\|h_{n}\right\| \leq 1 \mathrm{e}$

$$
\left|\widehat{h_{n}}(a)-\widehat{h_{n}}(b)\right|=\left|L\left(\widehat{g_{n}}(a)\right)-L\left(\widehat{g_{n}}(b)\right)\right| \longrightarrow|L(1)-L(r)|=2,
$$

o que nos leva novamente a uma contradição.

Definição 2.3.4 Sejam A uma álgebra uniforme e $a, b \in \mathcal{M}(A)$. Dizemos que a e b são equivalentes $(a \sim b)$ se $\psi(a, b)<1$.

Vamos mostrar que a relação defininida acima é uma relação de equivalência. De fato, $a \sim a$, pois $\|a-a\|=0<2$. 
Vamos supor que $a \sim b$. Temos que $\|a-b\|=\|b-a\|$. Portanto se $\|a-b\|<2$ então $\|b-a\|<2$. Logo $b \sim a$.

Por fim, se $a \sim b$ e $b \sim c$, vamos supor por absurdo que $a \nsim c$. Pelo Lema 2.3.3 existe uma sequência $\left(f_{n}\right) \subseteq A$ tal que $\left\|f_{n}\right\| \leq 1, \widehat{f}_{n}(a) \longrightarrow 1$ e $\widehat{f}_{n}(c)$ não converge para 1 . Como $a \sim b$, o Lema 2.3.3 garante que $\widehat{f}_{n}(b) \longrightarrow 1$. Agora, como $b \sim c$, novamente pelo Lema 2.3.3 temos que $\widehat{f}_{n}(c) \longrightarrow 1$, contradição. Portanto $a \sim c$, como queríamos.

Definição 2.3.5 Sejam A uma álgebra uniforme e a $\in \mathcal{M}(A)$. Chamamos de parte de Gleason de a o conjunto

$$
P(a)=\{b \in \mathcal{M}(A): a \sim b\} .
$$

Uma parte de Gleason é chamada de não-trivial se contiver no mínimo dois pontos.

Teorema 2.3.6 Seja A uma álgebra uniforme. As partes de Gleason de $\mathcal{M}(A)$ formam uma decomposição de $\mathcal{M}(A)$ em conjuntos disjuntos, onde cada um é união enumerável de conjuntos $w^{*}$-compactos.

Demonstração: Seja $a \in \mathcal{M}(A)$. Então temos que

$$
P(a)=\{b \in \mathcal{M}(A):\|a-b\|<2\}=\bigcup_{n \in \mathbb{N}}\left\{b \in \mathcal{M}(A):\|a-b\| \leq 2-2^{-n}\right\} .
$$

Agora, pelo Teorema 1.2.41 (Alaoglu), cada conjunto $\left\{b \in \mathcal{M}(A):\|a-b\| \leq 2-2^{-n}\right\}$ é $w^{*}$-compacto. Portanto segue o resultado.

Proposição 2.3.7 Sejam A uma álgebra uniforme e $a \in \mathcal{M}(A)$. Então $P(a) \subseteq \mathcal{M}(A)$ é aberta e fechada na topologia da norma.

Demonstração: É claro que $P(a)=\{b \in \mathcal{M}(A):\|a-b\|<2\}$ é aberto em $\mathcal{M}(A)$.

Para mostrar que $P(a)$ é fechado, vamos mostrar que seu complementar é aberto. Se $b \notin P(a)$ então $b \nsim a$ e consequentemente $P(b)$ e $P(a)$ são disjuntos. Logo $P(b)$ está contido em $\mathcal{M}(A) \backslash P(a)$ e como $P(b)$ é aberto, temos que o complementar de $P(a)$ é aberto, como queríamos. 
Teorema 2.3.8 Seja A uma álgebra uniforme. Se existirem um aberto conexo $\Omega \subseteq \mathbb{C} e$ uma aplicação $\phi: \Omega \longrightarrow \mathcal{M}(A)$ tal que $\hat{f} \circ \phi \in \mathcal{H}(\Omega)$, para toda $f \in A$, então $\phi(\Omega)$ está contido em alguma parte de Gleason de $\mathcal{M}(A)$.

Demonstração: Consideremos $a, b \in \phi(\Omega)$ e vamos supor por absurdo que $a \nsim b$, ou seja, que $\|a-b\|=2$. Então existe uma sequência $\left(f_{n}\right) \subseteq A$, $\left\|f_{n}\right\| \leq 1$ tal que

$$
\left|a\left(f_{n}\right)-b\left(f_{n}\right)\right| \longrightarrow 2 \text {, ou seja, }\left|\widehat{f}_{n}(a)-\widehat{f}_{n}(b)\right| \longrightarrow 2 \text {. }
$$

Sejam $\alpha, \beta \in \Omega$ tais que $\phi(\alpha)=a$ e $\phi(\beta)=b$.

Por hipótese $\widehat{f}_{n} \circ \phi: \Omega \longrightarrow \mathbb{C}$ é holomorfa em $\Omega$. Afirmamos que a família $\left\{\widehat{f}_{n} \circ \phi\right.$ : $n \in \mathbb{N}\}$ é limitada em $\Omega$. De fato, como $A$ é uma álgebra uniforme, o Teorema 2.2.6(1) garante que $\left\|\widehat{f}_{n}\right\|=\left\|f_{n}\right\|, \forall n \in \mathbb{N}$. Assim, para cada $z \in \Omega$ temos que

$$
\sup _{z \in \Omega}\left|\widehat{f}_{n} \circ \phi(z)\right|=\sup _{z \in \Omega}\left|\widehat{f}_{n}(\phi(z))\right| \leq\left\|\widehat{f}_{n}\right\|=\left\|f_{n}\right\| \leq 1 \text {. }
$$

Portanto, pelo Teorema 1.2.22, (Montel), existem uma subsequência $\left(f_{n_{k}}\right)$ de $\left(f_{n}\right)$ e $g \in$ $\mathcal{H}(\Omega)$ tais que $\widehat{f_{n_{k}}} \circ \phi$ converge para $g$ uniformemente sobre os compactos de $\Omega$.

$$
\begin{aligned}
& \text { Assim, }|g(\alpha)-g(\beta)|=\lim _{k \rightarrow \infty}\left|\widehat{f_{n_{k}}}(a)-\widehat{f_{n_{k}}}(b)\right|=2 \text {, ou seja, } 2=|g(\alpha)-g(\beta)| \leq \\
& |g(\alpha)|+|g(\beta)| .
\end{aligned}
$$

Como $|g(\alpha)| \leq 1$ e $|g(\beta)| \leq 1$, segue $|g(\alpha)|=|g(\beta)|=1$, ou seja, $|g|$ atinge seu máximo em $\alpha \in \Omega$. Como $\Omega$ é aberto e conexo, temos pelo Teorema 1.2 .9 que $g$ é constante. Mas isto é uma contradição pois $|g(\alpha)-g(\beta)|=2$, ou seja, $g(\alpha) \neq g(\beta)$.

Portanto existe $a \in \mathcal{M}(A)$ tal que $\phi(\Omega) \subseteq P(a)$.

A seguinte definição será utilizada no capítulo 4 .

Definição 2.3.9 Seja $A$ uma álgebra uniforme e suponha que existe $\Omega \subseteq \mathbb{C}$ como no Teorema 2.3.8. Dizemos que $\phi(\Omega)$ é um disco analítico de $\mathcal{M}(A)$.

Apresentaremos exemplos de partes de Gleason de elementos do espectro das álgebras uniformes $\mathcal{A}(D)$ e $\mathcal{H}^{\infty}(D)$ nos capítulos 3 e 4 , respectivamente, após termos calculado o espectro de tais álgebras. 


\section{Capítulo 3}

\section{A álgebra $\mathcal{A}(D)$}

Neste capítulo estudaremos operadores entre a álgebra $\mathcal{A}(D)$, da forma $u C_{\varphi}$. Apresentaremos caracterizações para a compacidade de tal operador. Por fim calcularemos o espectro do mesmo.

Pelo fato de $\mathcal{A}(D)$ ser uma álgebra de funções, muitas vezes neste texto estaremos nos referindo a elementos invertíveis de $\mathcal{A}(D)$ tanto como elementos invertíveis desta álgebra como funções invertíveis. Para não causar confusão, se $f \in \mathcal{A}(D)$ é invertível como elemento desta álgebra, diremos que $f$ é invertível. Se $f$ for invertível como uma função. diremos que $f$ é uma função ínvertível.

Os resultados apresentados neste capítulo foram originalmente demonstrados por $\mathrm{R}$. Aron, P. Galindo e M. Lindström em [1], e por H. Kamowitz no trabalho [19].

\subsection{Partes de Gleason de $\mathcal{M}(\mathcal{A}(D))$}

Nesta seção iremos calcular o espectro de $\mathcal{A}(D)$ e as partes de Gleason de elementos de $\mathcal{M}(\mathcal{A}(D))$.

Proposição 3.1.1 Seja $\phi: \mathcal{A}(D) \longrightarrow \mathbb{C}$ um homomorfismo não nulo. Então ф é uma avaliação. 
Demonstração: Seja $\phi: \mathcal{A}(D) \longrightarrow \mathbb{C}$ um homomorfismo não nulo. Vamos mostrar que existe $b \in \bar{D}$ tal que $o=\delta_{b}$. Pelo Teorema 2.1.6 temos que $\|\phi\|=1$. Considere $b=\phi(i d)$. Então $|b|=|\phi(i d)| \leq\|\phi\| \cdot\|i d\|=1$.

Sabemos que se $c \in \mathbb{C}$ entào $o(c)=c$. Para cada $n \in \mathbb{N}$ seja $q_{n}(z)=\sum_{i=1}^{n} a_{i} z^{i}$, onde $a_{i} \in \mathbb{C}, \forall i=1, \cdots, n$. Assim

$$
\phi\left(q_{n}\right)=\sum_{i=1}^{n} a_{i} \phi(i d)^{i}=\sum_{i=1}^{n} a_{i} b^{i} .
$$

Consequentemente temos que $\phi\left(q_{n}\right)=q_{n}(b), \forall n \in \mathbb{N}$. Pela Proposição 1.2.24, temos que para cada $f \in \mathcal{A}(D)$ existe uma sequência $\left(p_{n}\right)$ de polinômios tal que $p_{n} \longrightarrow f$ em $\mathcal{A}(D)$. Então é claro que

$$
p_{n}(b) \longrightarrow f(b) \text {. }
$$

Como $\phi(f)=\phi\left(f-p_{n}+p_{n}\right)=\phi\left(f-p_{n}\right)+\phi\left(p_{n}\right)=\phi\left(f-p_{n}\right)+p_{n}(b)$, segue que $\phi(f)-p_{n}(b)=\phi\left(f-p_{n}\right)$. E assim. $\left|\phi(f)-p_{n}(b)\right|=\left|\phi\left(f-p_{n}\right)\right| \leq\|\phi\| \cdot\left\|f-p_{n}\right\| \longrightarrow 0$, em $\mathcal{A}(D)$. Portanto

$$
p_{n}(b) \longrightarrow \phi(f)
$$

De (3.1) e (3.2) concluímos que $\phi(f)=f(b)$.

Como $f$ é um elemento arbitrário de $\mathcal{A}(D)$, concluímos que $\phi=\delta_{b}$, como queríamos.

Proposição 3.1.2 O espectro de $\mathcal{A}(D)$ é homeomorfo a $\bar{D}$.

Demonstração: Seja $\delta: \bar{D} \longrightarrow \mathcal{M}(\mathcal{A}(D))$ definida no Teorema 2.2.5. Segue diretamente do Teorema 2.2.5 e da Proposição 3.1 .1 que $\delta$ é um homeomorfismo sobre $\mathcal{M}(\mathcal{A}(D))$.

No que se segue, e quando for conveniente, não estaremos fazendo distinção entre números complexos $z \in \bar{D}$ e avaliações $\delta_{z} \in \mathcal{M}(\mathcal{A}(D))$.

Lema 3.1.3 Sejam $\delta_{a}, \delta_{b} \in \mathcal{M}(\mathcal{A}(D))$ tais que $a \in \bar{D}$ e $b \in D$ e $\psi\left(\delta_{a}, \delta_{b}\right)=\sup \left\{\left|\hat{f}\left(\delta_{a}\right)\right|\right.$ : $\left.f \in \mathcal{A}(D)_{\delta_{b}} e\|f\| \leq 1\right\}$. Então $\psi\left(\delta_{a}, \delta_{b}\right)=\left|\frac{a-b}{1-\tilde{b} a}\right|$.

Demonstração: Lembramos que $\mathcal{A}(D)_{\delta_{b}}=\left\{f \in \mathcal{A}(D): \hat{f}\left(\delta_{b}\right)=0\right\}$ e que $f(z)=\hat{f}\left(\delta_{z}\right)$, . $\forall z \in \bar{D}$. 
Seja $f \in \mathcal{A}(D)_{\delta_{b}}$ com $\|f\| \leq 1$. Então o Lema 1.2.17 (Schwarz-Pick) nos diz que

$$
|f(a)-f(b)| \leq|1-f(a) \overline{f(b)}|\left|\frac{a-b}{1-\bar{b} a}\right|
$$

ou seja

$$
|f(a)| \leq\left|\frac{a-b}{1-\bar{b} a}\right|
$$

Agora, como $f(a)=\hat{f}\left(\delta_{a}\right)$, segue que $\psi\left(\delta_{a}, \delta_{b}\right) \leq\left|\frac{a-b}{1-\bar{b} a}\right|$.

Consideremos $\varphi_{b}: \bar{D} \longrightarrow \bar{D}$ dada por $\varphi_{b}(z)=\frac{z-b}{1-\bar{b} z}$. Temos que $\varphi_{b} \in \mathcal{A}(D),\left\|\varphi_{b}\right\| \leq 1$ e $\varphi_{b} \in \mathcal{A}(D)_{\delta_{b}}$.

Logo $\left|\widehat{\varphi}_{b}\left(\delta_{a}\right)\right| \leq \psi\left(\delta_{a}, \delta_{b}\right)$, ou seja, $\left|\frac{a-b}{1-\overline{b a}}\right| \leq \psi\left(\delta_{a}, \delta_{b}\right)$, donde segue que $\psi\left(\delta_{a}, \delta_{b}\right)=$ $\left|\frac{a-b}{1-\bar{b} a}\right|$, como queríamos.

Proposição 3.1.4 Seja $\delta_{b} \in \mathcal{M}(\mathcal{A}(D))$. Então $P\left(\delta_{b}\right)=\left\{\delta_{b}\right\}$ se $|b|=1$ e $P\left(\delta_{b}\right)=\delta(D)$ se $|b|<1$.

Demonstração: Seja $\delta_{b} \in \mathcal{M}(\mathcal{A}(D))$ fixo com $|b|<1$ e consideremos a aplicação $\varphi_{b}$ definida no Lema acima. Pela Proposição 1.2.14, $\varphi_{b}(D)=D$ e $\varphi_{b}(\partial D)=\partial D$.

Se $|a|<1$, pelo Lema 3.1.3, $\psi\left(\delta_{a}, \delta_{b}\right)=\left|\varphi_{b}(a)\right|<1$ e pela Definição 2.3 .4 temos que $\delta_{a} \sim \delta_{b}$. Logo $\delta(D) \subseteq P\left(\delta_{b}\right)$. Agora, seja $\delta_{c} \in P\left(\delta_{b}\right)$. Vamos supor por absurdo que $|c|=1$. Então, pelo Lema 3.1 .3 , temos que $\psi\left(\delta_{c}, \delta_{b}\right)=\left|\varphi_{b}(c)\right|=1$, ou seja, $\delta_{c} \notin P\left(\delta_{b}\right)$, uma contradição. Logo $|c|<1$ e $P\left(\delta_{b}\right)=\delta(D)$.

Agora seja $\delta_{b} \in \mathcal{M}(\mathcal{A}(D))$ com $|b|=1$.

Seja $a$ tal que $|a|<1$. Pela Proposição 1.2.16, para cada $n \in \mathbb{N}$ existe um automorfismo $L_{n}$ tal que

$$
L_{n}(a)=-1+\frac{1}{2^{n}} \text { e } L_{n}(b)=1 .
$$

Seja $f_{n}=\frac{1}{2}\left(1-L_{n}\right)$. Então $f_{n} \in \mathcal{A}(D),\left\|f_{n}\right\| \leq 1, f_{n}(a)=\frac{1}{2}\left(1+1-\frac{1}{2^{n}}\right) \longrightarrow 1 \mathrm{e}$ $f_{n} \in \mathcal{A}(D)_{\delta_{b}}$. Assim, do Lema 2.3.3, segue que

$$
\psi\left(\delta_{a}, \delta_{b}\right)=1
$$


Seja agora $a$ tal que $|a|=1$ e $a \neq b$. Tomemos o automorfismo $L$ tal que $L(a)=-1$ e $L(b)=1$. Seja $f=\frac{1}{2}(1-L)$. Então $f \in \mathcal{A}(D),\|f\| \leq 1, f(a)=1$ e $f \in \mathcal{A}(D)_{\delta_{b}}$. Segue novamente do Lema 2.3 .3 que

$$
\psi\left(\delta_{a}, \delta_{b}\right)=1
$$

Portanto, de (3.3) e (3.4), concluímos que $\delta_{a} \nsim \delta_{b}, \forall a \in \bar{D}$; e $\operatorname{assim} P\left(\delta_{b}\right)=\left\{\delta_{b}\right\}$.

\subsection{Operadores de Composição de $\mathcal{A}(D)$ em $\mathcal{A}(D)$}

Definição 3.2.1 Seja $\varphi \in \mathcal{A}(D)$ tal que $\varphi(\bar{D}) \subseteq \bar{D}$. O operador $C_{\varphi}: \mathcal{A}(D) \longrightarrow \mathcal{A}(D)$ definido por

$$
C_{\varphi}(f)=f \circ \varphi, \forall f \in \mathcal{A}(D),
$$

é denominado de operador de composição.

É claro que o operador $C_{\varphi}$ definido acima é um homomorfismo contínuo, pois para cada $f, g \in \mathcal{A}(D)$ temos que $\left\|C_{\varphi}(f)-C_{\varphi}(g)\right\|=\sup _{z \in \partial D}|f(\varphi(z))-g(\varphi(z))| \leq\|f-g\|$.

Proposição 3.2.2 Seja $T: \mathcal{A}(D) \longrightarrow \mathcal{A}(D)$ um homomorfismo. Então $T$ é um operador de composição.

Demonstração: Seja $T: \mathcal{A}(D) \longrightarrow \mathcal{A}(D)$ um homomorfismo. Para cada $z \in \bar{D}$, seja $\phi: \mathcal{A}(D) \longrightarrow \mathbb{C}$ um homomorfismo complexo definido por $\phi=\delta_{z} \circ T$. Pela Proposição 3.1.1, existe um único $b \in \bar{D}$ tal que $\phi(f)=f(b), \forall f \in \mathcal{A}(D)$. Assim podemos definir $g: \bar{D} \longrightarrow \mathbb{C}$ por $g(z)=b$. Observamos que $g(\bar{D}) \subseteq \bar{D}$. Agora, como

$$
f(g(z))=f(b)=\phi(f)=\left(\delta_{z} \circ T\right)(f)=\delta_{z}(T(f))=T(f)(z)
$$

segue que $T(f)(z)=f(g(z)), \forall z \in \bar{D}, \forall f \in \mathcal{A}(D)$, isto é $T(f)=f \circ g, \forall f \in \mathcal{A}(D)$. Logo $T(i d)=g$, e como $T(i d) \in \mathcal{A}(D)$, segue que $g \in \mathcal{A}(D)$; e portanto $T$ é um operador de composição, como queríamos.

Proposição 3.2.3 Seja $\varphi \in \mathcal{A}(D)$ tal que $\varphi(\bar{D}) \subseteq \bar{D}$. Então $C_{\emptyset}: \mathcal{A}(D) \longrightarrow \mathcal{A}(D)$ é invertivel se e somente se $\varphi: \bar{D} \longrightarrow \bar{D}$ é uma função invertivel. 
Demonstração: Vamos supor que $C_{\varphi}$ é invertível. Como a função identidade $(i d)$ pertence a $\mathcal{A}(D)$, segue que existe $\psi \in \mathcal{A}(D)$ tal que $C_{\varphi}(\psi)=i d$, ou seja, $\psi \circ \varphi=i d$. Sejam $z, w \in \bar{D}$ tais que $\varphi(z)=\varphi(w)$. Então $z=i d(z)=\psi(\varphi(z))=\psi(\varphi(w))=i d(w)=w$, ou seja, $\varphi$ é injetora.

Vamos provar que $\varphi$ é sobrejetora. Para tal tomemos $w \in \bar{D}$. Queremos encontrar $z \in \bar{D}$ tal que $\varphi(z)=w$. Como $C_{\varphi}$ é invertivel, temos que $C_{\varphi}^{*}: \mathcal{A}(D)^{\prime} \longrightarrow \mathcal{A}(D)^{\prime}$ (o adjunto de $C_{\varphi}$ ) é invertível. Então existe um funcional linear $\phi \in \mathcal{A}(D)^{\prime}$ tal que $C_{\varphi}^{*}(\phi)=\delta_{w}$, onde $\delta_{w}$ é a avaliação no ponto $w$.

Afirmamos que $\phi \in \mathcal{M}(\mathcal{A}(D))$. Como $\phi \in \mathcal{A}(D)^{\prime}$, precisamos provar que $\phi(f \cdot g)=$ $\phi(f) \cdot \phi(g), \forall f, g \in \mathcal{A}(D)$. Com efeito, tomemos $f, g \in \mathcal{A}(D)$. Como $C_{\varphi}$ é invertível, existem $F, G, H \in \mathcal{A}(D)$ tais que $C_{\varphi}(F)=f, C_{\varphi}(G)=g$ e $C_{\varphi}(H)=f \cdot g$. Portanto, para todo $z \in \bar{D}$ temos que

$$
F(\varphi(z)) \cdot G(\varphi(z))=f(z) \cdot g(z)=(f \cdot g)(z)=H(\varphi(z))
$$

Agora, como $D$ é aberto e $\varphi$ é não constante, o Teorema 1.2.7, Aplicação Aberta, garante que $\varphi(D) \subseteq \bar{D}$ é aberto. ou seja, $\varphi(D) \subseteq D$. Por (3.5) temos que $(F \cdot G)(z)=H(z)$, $\forall z \in \varphi(D)$. Como $F \cdot G$ e $H$ são analíticas em $D$, segue do Corolário 1.2.3 (Princípio do Prolongamento Analítico), que $F \cdot G$ e $H$ coincidem em $D$. Agora, como $F \cdot G$ e $H$ são contínuas em $\bar{D}$, segue que $F \cdot G=H$. Portanto

$$
\begin{aligned}
& \phi(f \cdot g)=\phi\left(C_{\varphi}(F) \cdot C_{\varphi}(G)\right)=\phi\left(C_{\varphi}(F \cdot G)\right)=\phi\left(C_{\varphi}(H)\right)=C_{\varphi}^{*}(\phi)(H)=\delta_{w}(H)=H(w)= \\
& =F(w) \cdot G(w)=\delta_{w}(F) \cdot \delta_{w}(G)=C_{\varphi}^{*}(\phi)(F) \cdot C_{\varphi}^{*}(\phi)(G)=\phi\left(C_{\varphi}(F)\right) \cdot \phi\left(C_{\varphi}(G)\right)=\phi(f) \cdot \phi(g) .
\end{aligned}
$$

Portanto temos que $\phi \in \mathcal{M}(\mathcal{A}(D))$. Agora, como pela Proposição 3.1.1, todo elemento de $\mathcal{M}(\mathcal{A}(D))$ é uma avaliação, temos que existe $z \in \bar{D}$ tal que $\phi=\delta_{z}$. Logo

$$
w=\delta_{w^{\prime}}(i d)=C_{\varphi}^{*}\left(\delta_{z}\right)(i d)=\delta_{z}\left(C_{\varphi}(i d)\right)=\delta_{z}(\varphi)=\varphi(z),
$$

o que mostra que $\varphi$ é sobrejetora, como queríamos.

Reciprocamente, se $\varphi \in \mathcal{A}(D)$ é tal que $\varphi: \bar{D} \longrightarrow \bar{D}$ é uma função invertível, considere o operador de composição $C_{\varphi^{-1}}: \mathcal{A}(D) \longrightarrow \mathcal{A}(D)$. Então, para cada $f \in \mathcal{A}(D)$ temos que

$$
C_{\varphi^{-1}}\left(C_{\varphi}(f)\right)=C_{\varphi^{-1}}(f \circ \varphi)=f \circ \varphi \circ \varphi^{-1}=f
$$




$$
C_{\varphi}\left(C_{\varphi^{-1}}(f)\right)=C_{\varphi}\left(f \circ \varphi^{-1}\right)=f \circ \varphi^{-1} \circ \varphi=f,
$$

ou seja, $C_{\varphi^{-1}} \circ C_{\varphi}=I d=C_{\varphi} \circ C_{\varphi^{-1}}$. Portanto $C_{\varphi}$ é invertivel.

A próxima proposição apresenta uma caracterização para a compacidade e $w$-compacidade de um operador de composição $C_{\varphi}: \mathcal{A}(D) \longrightarrow \mathcal{A}(D)$. A demonstração é uma adaptação para a álgebra $\mathcal{A}(D)$ da apresentada em [1] para álgebras que são generalizações das álgebras clássicas.

Proposição 3.2.4 Sejam $\varphi \in \mathcal{A}(D)$ tal que $\varphi(\bar{D}) \subseteq \bar{D}, \varphi$ não constante e $C_{\varphi}: \mathcal{A}(D) \longrightarrow$ $\mathcal{A}(D)$ um operador de composição. As afirmações seguintes são equivalentes:

1. $C_{\varphi}$ é compacto;

2. $C_{\varphi}$ é fracamente compacto;

3. $\varphi(\bar{D}) \subset D$.

\section{Demonstração:}

(1) $\Rightarrow$ (2) Segue imediatamente da Proposição 1.2.57.

(2) $\Rightarrow$ (3) Sabemos que $\varphi(\bar{D}) \subseteq \bar{D}$. Queremos mostrar que $\varphi(\bar{D}) \subset D$. Vamos supor por absurdo que existe $z \in \bar{D}$ tal que $\varphi(z)=z_{0} \in \partial D$. Agora de $z \in \bar{D}$ temos que existe uma sequência $\left(z_{j}\right) \subseteq D$ tal que $z_{j} \longrightarrow z$. Portanto $\varphi\left(z_{j}\right) \longrightarrow \varphi(z)=z_{0}$.

Pelo Teorema 1.2.7 (Aplicação Aberta), temos que $\varphi$ é aberta, ou seja, $\varphi(D) \subseteq D$. $\operatorname{Logo}\left|\varphi\left(z_{j}\right)\right|<1, \forall j \in \mathbb{N}$.

Seja $l: \bar{D} \longrightarrow \mathbb{C}$ dada por $l(z)=\frac{z}{z_{0}}$. Então $l \in \mathcal{A}(D), l\left(z_{0}\right)=1$ e $\|l\|=1$.

Para cada $n \in \mathbb{N}$, vamos definir $l^{n}: \bar{D} \longrightarrow \mathbb{C}$ por $l^{n}(z)=(l(z))^{n}, \forall z \in \bar{D}$. É claro que $l^{n} \in \mathcal{A}(D), \forall n \in \mathbb{N}$ e que o conjunto $\left\{l^{n}: n \in N\right\} \subseteq \mathcal{A}(D)$ é limitado. Como $C_{\varphi}$ é fracamente compacto, $\left(C_{\varphi}\left(l^{n}\right)\right)$ admite uma subsequência $w$-convergente. Portanto existe $f \in \mathcal{A}(D)$ tal que, sem perda de generalidade

$$
C_{\varphi}\left(l^{n}\right)=l^{n} \circ \varphi \stackrel{w}{\longrightarrow} f .
$$

Para cada $z_{j}$, vamos considerar a avaliação $\delta_{z_{j}}$. Pela Observação 1.2.32:

$$
\lim _{n \rightarrow \infty} \delta_{z_{\jmath}}\left(l^{n} \circ \varphi\right)=\delta_{z_{j}}(f)
$$


Pelo Teorema 1.2.41 (Alaoglu), a sequência $\left(\delta_{z_{j}}\right)$ tem um $w^{*}$-ponto de acumulação $u \in$ $\mathcal{A}(D)^{\prime}$. Como $C_{\varphi}\left(l^{n}\right) \in \mathcal{A}(D)$, passando para uma subsequência de $\left(\delta_{z_{\jmath}}\right)$ segue da Observação 1.2 .37 que:

$$
\begin{gathered}
\lim _{j \rightarrow \infty} \delta_{z_{j}}\left(C_{\varphi}\left(l^{n}\right)\right)=u\left(C_{\varphi}\left(l^{n}\right)\right), \text { isto é } \\
\lim _{j \rightarrow \infty} \delta_{z_{j}}\left(l^{n} \circ \varphi\right)=u\left(l^{n} \circ \varphi\right) .
\end{gathered}
$$

Como para cada $n \in \mathbb{N}$ :

$$
\begin{gathered}
\lim _{j \rightarrow \infty} \delta_{z}\left(l^{n} \circ \varphi\right)=\lim _{j \rightarrow \infty} l^{n}\left(\varphi\left(z_{j}\right)\right)=l^{n}\left(z_{0}\right)=1, \text { segue que } \\
u\left(l^{n} \circ \varphi\right)=1 .
\end{gathered}
$$

Por outro lado, como $\left|\varphi\left(z_{j}\right)\right|<1$ e $\left|z_{0}\right|=1$, temos que

$$
l^{n}\left(\varphi\left(z_{j}\right)\right)=\left(\frac{\varphi\left(z_{j}\right)}{z_{0}}\right)^{n} \longrightarrow 0, n \rightarrow \infty .
$$

Logo, para cada $j \in \mathbb{N}$ :

$$
0=\lim _{n \rightarrow \infty} l^{n}\left(\varphi\left(z_{j}\right)\right)=\lim _{n \rightarrow \infty} \delta_{z_{j}}\left(l^{n} \circ \varphi\right)=\delta_{z_{j}}(f)
$$

Portanto

$$
0=\lim _{j \rightarrow \infty} \delta_{z_{j}}(f)=u(f)
$$

Agora, como $l^{n} \circ \varphi \stackrel{w}{\longrightarrow} f$, temos que $u\left(l^{n} \circ \varphi\right) \longrightarrow u(f)$, o que é uma contradição com (3.6) e (3.7).

(3) $\Rightarrow$ (1) Vamos supor que $C_{\varphi}$ não é compacto. Então existem $\epsilon>0$ e uma sequência $\left(f_{n}\right) \subseteq \mathcal{A}(D) \operatorname{com}\left\|f_{n}\right\| \leq 1, \forall n \in \mathbb{N}$ e $\left\|C_{\varphi}\left(f_{n}\right)-C_{\varphi}\left(f_{m}\right)\right\| \geq \epsilon, \forall n \neq m$. Como $\left\{f_{n}: n \in \mathbb{N}\right\}$ é uma família limitada, segue pelo Teorema 1.2.22 (Montel) que $\left\{f_{n}: n \in \mathbb{N}\right\}$ é uma família normal, e portanto existe uma subsequência $\left(f_{n_{k}}\right) \subseteq\left(f_{n}\right)$ convergindo uniformemente sobre os compactos de D. Se $k_{0} \neq k$ :

$$
\sup _{w \in \varphi(\bar{D})}\left|f_{n_{k_{0}}}(w)-f_{n_{k}}(w)\right|=\sup _{z \in \bar{D}}\left|f_{n_{k_{0}}}(\varphi(z))-f_{n_{k}}(\varphi(z))\right|=\left\|C_{\varphi}\left(f_{n_{k_{0}}}\right)-C_{\varphi}\left(f_{n_{k}}\right)\right\| \geq \epsilon
$$

o que é uma contradição com o fato de $\left(f_{n_{k}}\right)$ ser convergente sobre os compactos de $D$, já que $\varphi(\bar{D}) \subseteq D$ é compacto. Logo $C_{\varphi}$ é compacto, como queríamos.

É fácil produzir operadores de composição que não são compactos, como mostra o seguinte exemplo.

Exemplo 3.2.5 Se $\varphi(z)=\frac{z+1}{2}$, temos que $C_{\varphi}$ não é compacto, uma vez que $|\varphi(1)|=1$. 


\subsection{Operadores Compactos da Forma $u C_{\varphi}$}

Nesta seção estaremos estudando uma classe especial de operadores, que são os operadores da forma $u C_{\varphi}$. Tais operadores foram estudados em [19] por H. Kamowitz.

Sejam $u, \varphi \in \mathcal{A}(D), \operatorname{com} \varphi(\bar{D}) \subseteq \bar{D}$. Definimos $u C_{\varphi}: \mathcal{A}(D) \longrightarrow \mathcal{A}(D)$ da seguinte forma:

$$
u C_{\varphi}(f)(z)=u(z) \cdot f(\varphi(z)), \forall f \in \mathcal{A}(D), \forall z \in \bar{D} .
$$

Não é difícil ver que $u C_{\varphi}$ é um operador linear contínuo.

Se $u(z)=1, \forall z \in \bar{D}$, isto é, se $u$ é a função constante igual a 1 , temos que $u C_{\varphi}=C_{\varphi}$.

Se $\varphi(z)=z, \forall z \in \bar{D}$, isto é, se $\varphi$ é a identidade, temos que

$$
u C_{\varphi}(f)(z)=u(z) \cdot f(\varphi(z))=u(z) f(z)
$$

isto é, $u C_{\varphi}(f)=u \cdot f$ é um operador produto.

Se $\varphi \in \mathcal{A}(D),\|\varphi\| \leq 1$ e $n \in \mathbb{N}$ tal que $n \geq 2$, seja $\varphi^{n}: \bar{D} \longrightarrow \bar{D}$ a seguinte função

$$
\varphi^{n}(z)=\varphi\left(\varphi^{n-1}(z)\right), \forall z \in \bar{D}
$$

Lema 3.3.1 Sejam $u,,_{\uparrow} \in \mathcal{A}(D)$, com $\varphi(\bar{D}) \subseteq \bar{D}$. Então o operador $u C_{\varphi}$ é invertível se e somente se u é invertivel e $\varphi$ é uma função invertivel.

\section{Demonstração:}

Se $u C_{\varphi}$ é invertível, como $1 \in \mathcal{A}(D)$, existe $f \in \mathcal{A}(D)$ tal que $u(z) f(\varphi(z))=1$, $\forall z \in \bar{D}$. Logo $f \circ \varphi=u^{-1}$, ou seja, $u$ é invertível. Não é difícil verificar que o operador $u^{-1} \cdot u C_{\varphi}=C_{\varphi}$ é invertível. Pela Proposição 3.2 .3 , temos que $\varphi$ é uma função invertível.

Reciprocamente, vamos supor que $u$ é invertível e $\varphi$ é uma função invertível e provar que $u C_{\varphi}$ é invertivel. Para tal, considere o operador $\left(u^{-1} \circ \varphi^{-1}\right) C_{\varphi^{-1}}$. Vamos provar que $\left(u C_{\varphi}\right)^{-1}=\left(u^{-1} \circ \varphi^{-1}\right) C_{2^{-1}}$. Com efeito, se $f \in \mathcal{A}(D)$, temos que

$$
\begin{gathered}
u C_{\varphi}\left(\left(u^{-1} \circ \varphi^{-1}\right) C_{\varphi^{-1}}(f)\right)=u C_{\varphi}\left(\left(u^{-1} \circ \varphi^{-1}\right) \cdot\left(f \circ \varphi^{-1}\right)\right)= \\
\quad=u \cdot\left(u^{-1} \circ \varphi^{-1} \circ \varphi\right) \cdot\left(f \circ \varphi^{-1} \circ \varphi\right)=u \cdot u^{-1} \cdot f=f .
\end{gathered}
$$


Por outro lado $\left(\left(u^{-1} \circ \varphi^{-1}\right) C_{\varphi^{-1}}\right)\left(u C_{\varphi}(f)\right)=\left(\left(u^{-1} \circ \varphi^{-1}\right) C_{\varphi^{-1}}\right)(u \cdot f \circ \varphi)=$ $=\left(u^{-1} \circ \varphi^{-1}\right) \cdot\left(u \circ \varphi^{-1}\right) \cdot\left(f \circ \varphi \circ \varphi^{-1}\right)=1 \cdot f=f$.

Logo $u C_{\varphi}$ é invertível.

Lema 3.3.2 Sejam $u, \varphi \in \mathcal{A}(D)$, com $\varphi(\bar{D}) \subseteq \bar{D}$. Para cada $n \in \mathbb{N}$ tal que $n \geq 2 e$ $f \in \mathcal{A}(D)$ temos que

$$
\left(u C_{\varphi}\right)^{n}(f)=u \cdot u \circ \varphi \cdots \cdots \cdot u \circ \varphi^{n-1} \cdot f \circ \varphi^{n} .
$$

Demonstração: Vamos utilizar o Princípio da Indução Finita para demonstrar a igualdade acima.

Vamos provar a igualdade para $n=2$. Sejam $f \in \mathcal{A}(D)$ e $z \in \bar{D}$. Então

$\left(u C_{\varphi}\right)^{2}(f)(z)=\left(u C_{\varphi}(u \cdot f \circ \varphi)\right)(z)=u(z) \cdot u(\varphi(z)) \cdot f(\varphi(\varphi(z)))=u(z) \cdot u(\varphi(z)) \cdot f\left(\varphi^{2}(z)\right)$, ou seja $\left(u C_{\varphi}\right)^{2}(f)=u \cdot u \circ \varphi \cdot f \circ \varphi^{2}$.

Portanto o resultado vale para $n=2$. Vamos supor o resultado válido para $n$, e demonstrá-lo para $n+1$. Assim

$$
\begin{gathered}
\left(u C_{\varphi}\right)^{n+1}(f)=u C_{\varphi}\left(\left(u C_{\varphi}\right)^{n}(f)\right)=u C_{\varphi}\left(u \cdot u \circ \varphi \cdots \cdots u \circ \varphi^{n-1} \cdot f \circ \varphi^{n}\right)= \\
=u \cdot\left(u \cdot u \circ \varphi \cdots \cdots \circ \varphi^{n-1} \cdot f \circ \varphi^{n}\right) \circ \varphi=u \cdot\left(u \circ \varphi \cdot u \circ \varphi^{2} \cdots \cdots u \circ \varphi^{n} \cdot f \circ \varphi^{n+1}\right)= \\
=u \cdot u \circ \varphi \cdot u \circ \varphi^{2} \cdots \cdots u \circ \varphi^{n} \cdot f \circ \varphi^{n+1} .
\end{gathered}
$$

Portanto a igualdade vale para $n+1$. Logo, pelo Princípio da Indução Finita, concluímos que a igualdade vale para todo $n \in \mathbb{N}, n \geq 2$.

O próximo teorema apresenta uma caracterização para a compacidade de $u C_{\varphi}$. Sua demonstração foi apresentada por H. Kamowitz em [19].

Teorema 3.3.3 Suponha que $u, \varphi \in \mathcal{A}(D)$ e $\varphi(\bar{D}) \subseteq \bar{D}$.

1. Se $\varphi$ é uma função constante, então $u C_{\varphi}$ é um operador compacto. 
2. Se $\varphi$ não é uma função constante, então u $C_{\varphi}$ é um operador compacto se, e somente se, $|\varphi(z)|<1$ sempre que $u(z) \neq 0$.

\section{Demonstração:}

(1) Se $\varphi$ é constante, $\exists a \in \bar{D}$ tal que $\varphi(z)=a, \forall z \in \bar{D}$. Então se $z \in \bar{D}$ temos que $u C_{\varphi}(f)(z)=u(z) f(\varphi(z))=u(z) f(a)$, isto é, $u C_{\varphi}(f)=f(a) \cdot u . \operatorname{Assim} \operatorname{dim}\left(\operatorname{im}\left(u C_{\varphi}\right)\right)=1$, o que implica pelo Teorema 1.2.48 que $u C_{\varphi}$ é compacto.

(2) Vamos supor que $u C_{\varphi}: \mathcal{A}(D) \longrightarrow \mathcal{A}(D)$ é um operador compacto. Devemos provar que se $z \in \bar{D}$ e $u(z) \neq 0$ então $|\varphi(z)|<1$. Como $\varphi$ não é constante, o Teorema 1.2.7 (Aplicação Aberta) garante que $|\varphi(z)|<1$ sempre que $|z|<1$. Portanto é suficiente mostrar que $|\varphi(z)|<1$ se $u(z) \neq 0$ e $|z|=1$.

Vamos supor que tal afirmaçào é falsa, ou seja, que $\exists \theta$ tal que $u\left(e^{i \theta}\right) \neq 0$ e $\left|\varphi\left(e^{i \theta}\right)\right|=1$. Considere $\mu=\varphi\left(e^{i \theta}\right)$ e para cada inteiro positivo $n$ defina

$$
f_{n}(z)=\left(\frac{1}{2}(z+\mu)\right)^{n}
$$

É claro que $f_{n} \in \mathcal{A}(D)$ para cada $n \in \mathbb{N}$ e $\left\|f_{n}\right\|=1$, uma vez que para cada $z \in \bar{D}$

$$
\left|f_{n}(z)\right|=\left|\left(\frac{1}{2}(z+\mu)\right)^{n}\right|=\left|\left(\frac{1}{2}(z+\mu)\right)\right|^{n} \leq\left(\frac{1}{2}(|z|+|\mu|)\right)^{n} \leq 1,
$$

e $f_{n}$ atinge a norma em $\mu, \forall n \in \mathbb{N}$.

Como por hipótese $u C_{\varphi}$ é compacto, então existe uma subsequência $\left(f_{n_{k}}\right)$ de $\left(f_{n}\right)$ e uma função $F \in \mathcal{A}(D) \operatorname{com} u C_{\varphi}\left(f_{n_{k}}\right) \longrightarrow F$ em $\mathcal{A}(D)$, ou seja,

$$
u(z)\left(\frac{1}{2}(\varphi(z)+\mu)\right)^{n_{k}} \longrightarrow F(z), \forall z \in \bar{D} .
$$

Se $|z|<1$ então $|\varphi(z)|<1$. Consequentemente $\left|\frac{1}{2}(\varphi(z)+\mu)\right|<1 \mathrm{e}$

$$
\left(\frac{1}{2}(\varphi(z)+\mu)\right)^{n_{k}} \longrightarrow 0 .
$$

Assim $F(z)=0$, para $|z|<1$. No entanto, como $F$ é contínua em $\bar{D}$, temos que $F(z)=0, \forall z \in \bar{D}$, ou seja, $F=0$. Então $u C_{\varphi}\left(f_{n_{k}}\right) \longrightarrow 0 \mathrm{em} \mathcal{A}(D)$.

Em particular, $u C_{\varphi}\left(f_{n_{k}}\right)\left(e^{i \theta}\right) \longrightarrow 0$, ou seja, $u\left(e^{i \theta}\right)\left(\frac{1}{2}\left(\varphi\left(e^{i \theta}\right)+\mu\right)^{n_{k}} \longrightarrow 0\right.$. Mas para todo $k$ temos que

$$
\mid u\left(e^{i \theta}\right)\left(\frac{1}{2}\left(\varphi\left(e^{i \theta}\right)+\mu\right)^{n_{k}}|=| u\left(e^{i \theta}\right) \mid \neq 0\right.
$$


o que nos leva a uma contradição. Logo, se $u C_{\varphi}$ é compacto temos que $|\varphi(z)|<1$ sempre que $u(z) \neq 0$.

Reciprocamente, se $|\varphi(z)|<1$ sempre que $u(z) \neq 0$, para mostrar que $u C_{\varphi}$ é compacto, considere $\left(f_{n}\right) \subseteq \mathcal{A}(D)$ com $\left\|f_{n}\right\| \leq 1$. A família $\left\{f_{n}: n \in \mathbb{N}\right\}$ é limitada em $\bar{D}$ e portanto é uma família normal pelo Teorema 1.2.22 (Montel). Logo existem uma subsequência $\left(f_{n_{k}}\right) \subseteq\left(f_{n}\right)$ e uma função $g \in \mathcal{H}(D)$ tal que $f_{n_{k}} \longrightarrow g$ uniformemente sobre os compactos de $D$. Observemos que esta convergência implica que

$$
\sup _{|w|<1}|g(w)| \leq 1
$$

Vamos definir uma função $G$ em $\bar{D}$ da seguinte maneira:

$$
G(z)=\left\{\begin{array}{ll}
0 & |z|=1 \text { e } u(z)=0 \\
u(z) \cdot g(\varphi(z)) & |z|<1 \text { ou } u(z) \neq 0
\end{array} .\right.
$$

Se $|z|<1$ ou $u(z) \neq 0$ então $|\varphi(z)|<1$. Logo $G$ está bem definida.

Vamos mostrar que $G \in \mathcal{A}(D)$ e que $u C_{\varphi}\left(f_{n_{k}}\right) \longrightarrow G$ em $\mathcal{A}(D)$.

Se $|z|<1$ ou $u(z) \neq 0$ temos que $G$ é produto de funções contínuas.

Se $\left|z^{*}\right|=1$ e $u\left(z^{*}\right)=0$, seja $\left(z_{m}\right) \subseteq D$ com $z_{m} \longrightarrow z^{*}$. Para cada $m$, temos que $G\left(z_{m}\right)=u\left(z_{m}\right) g\left(\varphi\left(z_{m}\right)\right)$. Como $\left|g\left(\varphi\left(z_{m}\right)\right)\right| \leq 1, \forall m$ e $u\left(z_{m}\right) \longrightarrow u\left(z^{*}\right)=0$, temos que $G\left(z_{m}\right) \longrightarrow 0=G\left(z^{*}\right)$ e portanto $G$ é contínua em $\bar{D}$.

$G$ é analítica em $D$, pois $u$ e $(g \circ \varphi)$ o são. Então $G \in \mathcal{A}(D)$.

Para mostrar que $u C_{\varphi}\left(f_{n_{k}}\right) \longrightarrow G$ em $\mathcal{A}(D)$, vamos dividir $\bar{D}$ em três conjuntos, e mostrar a convergência em cada um deles.

Seja $V=\left\{e^{i \theta}: u\left(e^{i \theta}\right)=0\right\}$ e tome $\epsilon>0$. Afirmamos que existe aberto $U$ tal que $V \subseteq U$ e $|u(t)|<\epsilon, \forall t \in U$. De fato, como $u$ é contínua, para cada $\theta$ tal que $e^{i \theta} \in V$ e para cada $\epsilon>0$, existe um aberto $U_{\theta}$ tal que se $z \in U_{\theta}$, então $u(z) \in D_{\epsilon}\left(u\left(e^{i \theta}\right)\right)$. Portanto basta tomarmos

$$
U=\bigcup_{e^{i \theta} \in V} U_{\theta}
$$

Se $z \notin U$ então $u(z) \neq 0$ e portanto $|\varphi(z)|<1$. Logo $\varphi(\bar{D} \backslash U) \subseteq D$. Como $\bar{D} \backslash U$ é compacto, $\varphi(\bar{D} \backslash U)$ é compacto. Então, como $f_{n_{k}} \longrightarrow g$ uniformemente sobre os 
compactos de $D$, temos que

$$
f_{n_{k}}(\hat{\varphi}(z)) \longrightarrow g(\varphi(z)) \text { para } z \notin U \text {. }
$$

Logo $u(z) f_{n_{k}}(\varphi(z)) \longrightarrow u(z) g(\varphi(z)), \forall z \notin U$. Desta forma existe inteiro $N$ tal que

$$
\left|u(z) f_{n_{k}}(\varphi(z))-u(z) g(\varphi(z))\right|=\left|u C_{\varphi}\left(f_{n_{k}}\right)(z)-G(z)\right|<\epsilon,
$$

para todo $k \geq N$ e todo $z \notin U$. Portanto temos a convergência em $\bar{D} \backslash U$.

Agora, se $z \in U \backslash V$ e $k$ é qualquer:

$$
\begin{aligned}
& \left|u C_{\varphi}\left(f_{n_{k}}\right)(z)-G(z)\right|=\left|u(z) f_{n_{k}}(\varphi(z))-u(z) g(\varphi(z))\right| \leq \\
& \sup _{z \in U \backslash V}|u(z)|\left|f_{n_{k}}(\varphi(z))-g(\varphi(z))\right| \leq \epsilon\left(\left\|f_{n_{k}}\right\|+\|g\|\right) \leq 2 \epsilon,
\end{aligned}
$$

o que verifica a convergência no conjunto $U \backslash V$.

Finalmente, se $z \in V$, então $u C_{\varphi}\left(f_{n_{k}}\right)(z)=u(z) f_{n_{k}}(\varphi(z))=0=G(z)$.

Portanto, dado $\epsilon>0$, existe inteiro $N^{\prime}$ tal que $\left|u C_{\varphi}\left(f_{n_{k}}\right)(z)-G(z)\right|<2 \epsilon, k \geq N^{\prime} \mathrm{e}$ $\forall z \in \bar{D}$. Ou seja, $\left(u C_{\varphi}\right)\left(f_{n_{k}}\right) \longrightarrow G$ em $\mathcal{A}(D)$. Logo, se $|\varphi(z)|<1$ sempre que $u(z) \neq 0$, temos que $u C_{\varphi}$ é compacto.

Observação 3.3.4 Na Proposição 3.2 .4 provamos que um operador de composição $C_{\varphi}$ é compacto se e somente se $\varphi(\bar{D}) \subset D$. Vamos a seguir verificar que tal equivalência é precisamente o Teorema 3.3.3 acima demonstrado, no caso $u=1$.

Como $u=1$, o Teorema 3.3.3 garante que $C_{\varphi}$ é compacto se, e somente se, $|\varphi(z)|<1$, $\forall z \in \bar{D}$, ou seja, se e somente se $\varphi(\bar{D}) \subset D$.

\subsection{Espectro do Operador Compacto $u C_{\varphi}$}

Nesta seção calcularemos o espectro do operador $u C_{\varphi}$, quando $u C_{\varphi}$ é compacto. O cálculo do espectro de $u C_{\varphi}$ foi feito por H. Kamowitz em [19]. 
Observação 3.4.1 A demonstração da próxima proposição será dividida em itens. Em um dos itens e também na proposição subsequente, precisaremos trabalhar com representações de séries de potências. Estaremos usando $r_{i}$ para indicar qualquer série da forma

$$
r_{i}(z)=\sum_{n \geq i} a_{n} z^{n},
$$

ou seja, série cujo primeiro coeficiente não nulo é $a_{i}$.

Por abuso de notação. estaremos denotando por $r_{i}$ séries cujo primeiro coeficiente não nulo é o $i$-ésimo, independente dos coeficientes das séries. Ou seja, estaremos interessados em avaliar o índice $i$ no qual tem-se o primeiro coeficiente não nulo da série, e não nos valores de $a_{n}, n \geq i$, pois os mesmos não estarão interferindo nos cálculos a serem feitos.

Quando uma série da forma $r_{i}$ estiver sendo calculada em outra série da mesma forma, será usado o Binômio de Newton para estimar o índice do primeiro coeficiente não nulo da série resultante.

Proposição 3.4.2 Suponha que $u, \varphi \in \mathcal{A}(D), \varphi(\bar{D}) \subseteq \bar{D}$ e $\varphi(0)=0$. Então $u(0) \in$ $\sigma\left(u C_{\varphi}\right)$ e $u(0) \varphi^{\prime}(0)^{n} \in \sigma\left(u C_{\varphi}\right), \forall n \in \mathbb{N}, n \geq 1$.

Demonstração: Vamos provar inicialmente que $u(0) \in \sigma\left(u C_{\varphi}\right)$. Vamos supor por absurdo que $u(0) \notin \sigma\left(u C_{\varphi}\right)$, ou seja que $u C_{\varphi}-u(0) \cdot I d$ é invertível.

Logo, como $1 \in \mathcal{A}(D), \exists f \in \mathcal{A}(D)$ tal que $u(z) f(\varphi(z))-u(0) f(z)=1, \forall z \in \bar{D}$.

Para $z=0$ teríamos $u(0) f(0)-u(0) f(0)=1$, uma contradição. Consequentemente $u(0) \in \sigma\left(u C_{\varphi}\right)$.

Para demonstrar que $u(0) \varphi^{\prime}(0)^{n} \in \sigma\left(u C_{\varphi}\right)$, vamos analisar dois casos: quando $\varphi^{\prime}(0)=$ 0 e quando $u(0) \varphi^{\prime}(0) \neq 0$.

(i) Se $\varphi^{\prime}(0)=0$, o Teorema 1.2.8(3) garante que $\varphi$ não é uma função injetora e portanto não é uma função invertível. Assim, pelo Lema 3.3.1, o operador $u C_{\varphi}$ não é invertível e portanto $0=u(0) \varphi^{\prime}(0)^{n} \in \sigma\left(u C_{\varphi}\right), \forall n \in \mathbb{N}$.

(ii) Vamos supor que $u(0) \varphi^{\prime}(0) \neq 0$ e provar que $u(0) \varphi^{\prime}(0)^{n} \in \sigma\left(u C_{\varphi}\right), \forall n \geq 1$.

Se $\varphi^{\prime}(0)^{n}=1$ então $u(0) \varphi^{\prime}(0)^{n}=u(0)$, que já provamos pertencer ao espectro de $u C_{\varphi}$. No que se segue, portanto. estaremos supondo $\varphi^{\prime}(0)^{n} \neq 1, \forall n \in \mathbb{N}$. 
Para provar que $u(0) \varphi^{\prime}(0)^{n} \in \sigma\left(u C_{\varphi}\right)$ é preciso mostrar que $u(0) \varphi^{\prime}(0)^{n} \cdot I d-u C_{\varphi}$ não é invertível. Assim basta mostrar que $\xi(z)=z^{n}$ não pertence à imagem de $u(0) \varphi^{\prime}(0)^{n} \cdot I d-$ $u C_{\varphi}, \forall n \in \mathbb{N}$. Vamos supor por absurdo que existem $n \in \mathbb{N}$ e $f \in \mathcal{A}(D)$ tais que

$$
u(0) \varphi^{\prime}(0)^{n} f(z)-u(z) f(\varphi(z))=z^{n}, \forall z \in \bar{D}
$$

Para $z=0$ temos

$$
\begin{gathered}
u(0) \varphi^{\prime}(0)^{n} f(0)-u(0) f(0)=0, \text { ou seja }, \\
u(0)\left(f(0) \varphi^{\prime}(0)^{n}-f(0)\right)=0
\end{gathered}
$$

Como $u(0) \neq 0$ e $\varphi^{\prime}(0)^{n} \neq 1$ segue que $f(0)=0$. Portanto, de acordo com a Proposição 1.2.4, existem $m \in \mathbb{N}$ e $f_{0} \in \mathcal{H}(D)$ tais que $f_{0}(0) \neq 0$ e $f(z)=z^{m} f_{0}(z), \forall z \in D$.

Pelo Teorema 1.2.1, podemos escrever $f_{0}(z)=f_{0}(0)+r_{1}(z), u(z)=u(0)+r_{1}(z)$ e $\varphi(z)=\varphi^{\prime}(0) z+r_{2}(z)$. Então

$$
u(0) \varphi^{\prime}(0)^{n} f(z)-u(z) f(\varphi(z))=z^{n}
$$

é equivalente a

$$
\begin{gathered}
u(0) \varphi^{\prime}(0)^{n} z^{m}\left(f_{0}(0)+r_{1}(z)\right)-\left(u(0)+r_{1}(z)\right)\left(\varphi(z)^{m} f_{0}(\varphi(z))=z^{n}\right. \\
u(0) \varphi^{\prime}(0)^{n} z^{m}\left(f_{0}(0)+r_{1}(z)\right)-\left(u(0)+r_{1}(z)\right) \varphi(z)^{m}\left(f_{0}(0)+r_{1}(\varphi(z))=z^{n}\right. \\
u(0) \varphi^{\prime}(0)^{n} z^{m}\left(f_{0}(0)+r_{1}(z)\right)-\left(u(0)+r_{1}(z)\right)\left(\varphi^{\prime}(0) z+r_{2}(z)\right)^{m}\left(f_{0}(0)+r_{1}\left(\varphi^{\prime}(0) z+r_{2}(z)\right)=z^{n}\right. \\
u(0) \varphi^{\prime}(0)^{n} z^{m} f_{0}(0)+r_{m+1}(z)-\left(u(0)+r_{1}(z)\right)\left(\rho^{\prime}(0) z^{m}+r_{m+1}(z)\right)\left(f_{0}(0)+r_{1}(z)\right)=z^{n} \\
u(0) \varphi^{\prime}(0)^{n} z^{m} f_{0}(0)+r_{m+1}(z)-\left(u(0) \varphi^{\prime}(0)^{m} z^{m}+r_{m+1}(z)\right)\left(f_{0}(0)+r_{1}(z)\right)=z^{n} \\
u(0) \varphi^{\prime}(0)^{n} z^{m} f_{0}(0)+r_{m+1}(z)-u(0) \varphi_{\varphi^{\prime}}(0)^{m} z^{m} f_{0}(0)-r_{m+1}(z)=z^{n} .
\end{gathered}
$$

E finalmente ficamos com

$$
\left(u(0) \varphi^{\prime}(0)^{n} f_{0}(0)-u(0) \varphi^{\prime}(0)^{m} f_{0}(0)\right) z^{m}+r_{m+1}(z)=z^{n} .
$$

Se $m=n$, o lado esquerdo de (3.8) tem $n+1$ como índice do primeiro coeficiente não nulo, enquanto que do lado direito tal índice é $n$, uma contradição. Se $m \neq n$, o lado esquerdo de (3.8) tem $m$ como índice do primeiro coeficiente não nulo e o lado direito tem $n$, o que também é uma contradição. Logo segue o resultado. 
Proposição 3.4.3 Sejam $u, \varphi \in \mathcal{A}(D)$ tais que $\varphi$ não é constante, $\varphi(\bar{D}) \subseteq \bar{D}$ e $\varphi(0)=0$. Se $\lambda$ é um autovalor de $u C_{\varphi}$, então $\lambda \in\left\{u(0) \varphi^{\prime}(0)^{n}: n \in \mathbb{N}, n \geq 1\right\} \cup\{0, u(0)\}$.

Demonstração: Se $u=0$ então $u C_{\varphi}=0$. Neste caso, $\lambda=0$ é o único autovalor de $u C_{\varphi}$. Suponhamos $u \neq 0$. Seja $\lambda$ um autovalor de $u C_{\varphi}$ com $f$ autovetor correspondente. Logo $u C_{\varphi}(f)=\lambda f$. Se $\lambda=0$, teremos que $u \cdot f \circ \varphi=0$. Pelo Corolário 1.2.6. $\mathcal{A}(D)$ é um anel de integridade. Como $u \neq 0$ segue que $f=0$, um absurdo. Assim $\lambda \neq 0$.

Usando o Teorema 1.2.1, podemos escrever

$$
\begin{aligned}
& f(z)=a z^{m}+r_{m+1}(z), a \neq 0 \text { e } m \geq 0 \\
& u(z)=b z^{r}+r_{r+1}(z), b \neq 0 \text { e } r \geq 0 \text { e } \\
& \varphi(z)=c z^{s}+r_{s+1}(z), c \neq 0 \text { e } s \geq 1 .
\end{aligned}
$$

E assim substituindo na equação $\lambda f=u C_{\varphi}(f)$, temos que

$$
\lambda\left(a z^{m}+r_{m+1}(z)\right)=\left(b z^{r}+r_{r+1}(z)\right)\left(a\left(c z^{s}+r_{s+1}(z)\right)^{m}+r_{m+1}(z)\right),
$$

ou seja,

$$
\begin{gathered}
\lambda a z^{m}+r_{m+1}(z)=\left(b z^{r}+r_{r+1}(z)\right)\left(a\left(c^{m} z^{s m}+r_{s m+1}(z)\right)+r_{m+1}(z)\right) \\
\left.\lambda a z^{m}+r_{m+1}(z)=\left(b z^{r}+r_{r+1}(z)\right)\left(a c^{m} z^{s m}+r_{s m+1}(z)\right)+r_{m+1}(z)\right) \\
\lambda a z^{m}+r_{m+1}(z)=\left(b z^{r}+r_{r+1}(z)\right)\left(a c^{m} z^{s m}+r_{m+1}(z)\right)= \\
=a b c^{m} z^{r+s m}+r_{r+m+1}(z)+r_{r+1+s m}(z)+r_{r+m+2}(z)= \\
a b c^{m} z^{r+s m}+r_{r+m+1}(z) .
\end{gathered}
$$

Igualando potências, temos que $m=s m+r, m+1=r+m+1$ e $\lambda a=a b c^{m}$. Assim, temos que $\lambda=b c^{m}$ e

(i) $r=0$ e $s=1$ ou (ii) $m=0$ e $r=0$.

Vamos substituir os valores de $r, s$ e $m$ em ambos os casos.

(i) Para $r=0$ e $s=1$ temos que $u(z)=b+r_{1}(z)$ e $\operatorname{assim} u(0)=b$; e $\varphi(z)=c z+r_{2}(z)$, $\operatorname{com} \varphi^{\prime}(0)=c$. Dai $\lambda=u(0) \varphi^{\prime}(0)^{m}$.

(ii) Para $m=0$ e $r=0$ temos que $\lambda=b c^{m}=u(0)$.

Portanto temos que $\lambda \in\left\{u(0) \varphi^{\prime}(0)^{n}: n \in \mathbb{N}, n \geq 1\right\} \cup\{0, u(0)\}$, como queríamos. 
Corolário 3.4.4 Suponha $u, \varphi \in \mathcal{A}(D)$, $\varphi$ não é constante, $\varphi(\bar{D}) \subseteq \bar{D}$ e $\varphi(0)=0$. Se $u C_{\varphi}$ é compacto, então $\sigma\left(u C_{\varphi}\right)=\left\{u(0) \varphi^{\prime}(0)^{n}: n \in \mathbb{N}, n \geq 1\right\} \cup\{0, u(0)\}$.

Demonstração: Como $u C_{\varphi}$ é compacto, pelo Teorema 2.1.15 temos que todo elemento não nulo de $\sigma\left(u C_{\varphi}\right)$ pertence a $\operatorname{Aut}\left(u C_{\varphi}\right)$. Como, pelas Proposições 3.4 .2 e 3.4 .3 temos que

$$
\operatorname{Aut}\left(u C_{\varphi}\right) \subseteq\left\{u(0) \varphi^{\prime}(0)^{n}: n \in \mathbb{N}, n \geq 1\right\} \cup\{0, u(0)\} \subseteq \sigma\left(u C_{\varphi}\right),
$$

consequentemente segue que $\sigma\left(u C_{\varphi}\right)=\left\{u(0) \varphi^{\prime}(0)^{n}: n \in \mathbb{N}, n \geq 1\right\} \cup\{0, u(0)\}$, como queríamos demonstrar.

Definição 3.4.5 Seja $L: \bar{D} \longrightarrow \bar{D}$ um automorfismo. Dizemos que $L$ é elíptico se $L$ tiver dois pontos fixos: um em $D$ e outro fora de $\bar{D}$.

Observação: Vale ressaltar que o automorfismo $L=\lambda \varphi_{\alpha}(|\lambda|=1$ e $\alpha \in D)$ está definido em todo $\mathbb{C} \backslash\left\{\frac{1}{\bar{\alpha}}\right\}$. Por isso podemos falar em pontos fixos fora de $\bar{D}$, como na Definição 3.4 .5 .

Seja $L$ um automorfismo elíptico. Então existem $z_{0} \in D$ e $z_{1} \in \mathbb{C}_{\infty} \backslash \bar{D}$ tais que $L\left(z_{0}\right)=z_{0}$ e $L\left(z_{1}\right)=z_{1}$. Consideremos a aplicação $\varphi_{z_{0}}: D \longrightarrow D$ dada por

$$
\varphi_{z_{0}}(z)=\frac{z-z_{0}}{1-\overline{z_{0}} z}
$$

Temos que $\varphi\left(z_{0}\right)=0$ e pela Proposição 1.2.14 que $\left(\varphi_{z_{0}}\right)^{-1}=\varphi_{-z_{0}}$. Então a aplicação

$$
T=\varphi_{z_{0}} \circ L \circ \varphi_{-z o}: D \longrightarrow D
$$

é uma bijeção analítica de $D$ sobre $D$ e $T(0)=0$. Portanto, pelo Teorema 1.2.15 temos que $T(z)=\lambda \varphi_{0}(z)=\lambda z, \forall z \in D$, onde $\lambda$ é tal que $|\lambda|=1$.

Finalmente, de $L(z)=\varphi_{-z_{0}}\left(\lambda \varphi_{z_{0}}(z)\right)$, não é difícil ver que $\lambda=L^{\prime}\left(z_{0}\right)$ e portanto podemos concluir que se $L$ é um automorfismo elíptico e $z_{0} \in D$ é seu ponto fixo, então existe $\lambda \in \partial D$ tal que $L=\varphi_{-z_{0}} \circ T \circ \varphi_{z_{0}}$, onde $T(z)=\lambda z, \forall z \in D$ e $\lambda=L^{\prime}\left(z_{0}\right)$.

O seguinte teorema será útil no cálculo do espectro de $u C_{\varphi}$, porém não o demonstraremos. 
Teorema 3.4.6 (Teorema de Denjoy-Wolff) Seja $\varphi \in \mathcal{H}(D)$ tal que $\varphi(D) \subseteq D ; \varphi$ não é a identidade e nem um automorfismo elíptico. Então existe $z_{0} \in \bar{D}$ tal que $\varphi^{n} \longrightarrow z_{0}$ uniformemente sobre os compactos de D.

Demonstração: Sugerimos [7], pg. 58.

Observação: Seja $\varphi$ como no Teorema 3.4.6. Se $\varphi \in \mathcal{A}(D)$ então $\varphi\left(z_{0}\right)=z_{0}$ e neste caso $z_{0}$ é chamado de ponto fixo de Denjoy-Wolff de $\varphi$.

A seguir apresentaremos um exemplo de um automorfismo elíptico $\varphi$ tal que $\varphi^{n}$ não converge.

Exemplo 3.4.7 Seja $\varphi$ definida por $\varphi(z)=i z$, temos que $\varphi$ é um automorfismo elíptico e que

$$
\begin{aligned}
\varphi^{2}(z) & =-z: \\
\varphi^{3}(z) & =-\mathrm{i} z \\
\varphi^{4}(z) & =z \\
\varphi^{5}(z) & =\mathrm{i} z=\varphi(z) .
\end{aligned}
$$

Ou seja, $\left(\varphi^{n}(z)\right)$ oscila e portanto não tem limite. Logo o Teorema de Denjoy-Wolff realmente deve excluir os automorfismos elípticos.

Não é difícil ver que se $\varphi(z)=z$ e $u C_{\varphi}$ é compacto, então $u C_{\varphi}=0$. E neste caso, 0 é o único valor espectral de $u C_{\varphi}$. Daqui em diante, estaremos supondo que $\varphi$ não é a identidade e nem um automorfismo elíptico.

Teorema 3.4.8 Sejam $u, \varphi \in \mathcal{A}(D), \operatorname{com} \varphi(\bar{D}) \subseteq \bar{D}$ e $u C_{\varphi}: \mathcal{A}(D) \longrightarrow \mathcal{A}(D)$ um operador compacto. Suponha que $z_{0}$ é o ponto fixo de Denjoy-Wolff de $\varphi$.

1. Se $\varphi$ é constante, então $\sigma\left(u C_{\varphi}\right)=\left\{0, u\left(z_{0}\right)\right\}$.

2. Se $\varphi$ não é constante e $\left|z_{0}\right|=1$, então $\sigma\left(u C_{\varphi}\right)=\{0\}$.

3. Se $\varphi$ não é constante e $\left|z_{0}\right|<1$, então $\sigma\left(u C_{\varphi}\right)=\left\{u\left(z_{0}\right) \varphi^{\prime}\left(z_{0}\right)^{n}: n \in \mathbb{N}, n \geq\right.$ $1\} \cup\left\{0, u\left(z_{0}\right)\right\}$. 


\section{Demonstração:}

(1) Se $\varphi$ é constante temos que $\varphi(z)=z_{0}, \forall z \in \bar{D}$. Vamos mostrar que 0 e $u\left(z_{0}\right)$ são autovalores de $u C_{\varphi}$.

De fato, se $F(z)=z-z_{0}$ então $u C_{\varphi}(F)(z)=u(z) F(\varphi(z))=u(z)\left(\varphi(z)-z_{0}\right)=$ $u(z)\left(z_{0}-z_{0}\right)=0 . \operatorname{Logo} 0 \in \operatorname{Aut}\left(u C_{\varphi}\right)$.

Por outro lado. $u C_{q}(u)(z)-u\left(z_{0}\right) u(z)=u(z) u(\varphi(z))-u\left(z_{0}\right) u(z)=u\left(z_{0}\right) u(z)-$ $u\left(z_{0}\right) u(z)=0$. Portanto $u\left(z_{0}\right) \in \operatorname{Aut}\left(u C_{\varphi}\right)$.

Como a imagem de $u C_{\varphi}$ tem dimensão 1, a Proposição 2.1.11 garante que $\operatorname{Aut}\left(u C_{\varphi}\right)$ deve conter no máximo dois elementos, ou seja, $\operatorname{Aut}\left(u C_{\varphi}\right)=\left\{0, u\left(z_{0}\right)\right\}$. Por fim, pela Proposição 2.1.13 e pelo Teorema 2.1.15, concluímos que $\sigma\left(u C_{\varphi}\right)=\left\{0, u\left(z_{0}\right)\right\}$.

(2) Como $u C_{\varphi}$ é compacto e $\left|\vartheta\left(z_{0}\right)\right|=\left|z_{0}\right|=1$, segue do Teorema 3.3 .3 que $u\left(z_{0}\right)=0$.

Para mostrar que $\sigma\left(u C_{\varphi}\right)=\{0\}$, vamos verificar que o raio espectral de $u C_{\varphi}$ é zero. Ou equivalentemente. pela Proposição 2.1.9, vamos mostrar que

$$
\lim _{n \rightarrow \infty}\left\|\left(u C_{\varphi}\right)^{n}\right\|^{\frac{1}{n}}=0
$$

Para tal, vamos dividir $\bar{D}$ em dois conjuntos e calcular o limite acima em cada um deles.

Sejam $V=\left\{e^{i \theta}: u\left(e^{i \theta}\right)=0\right\}$ e $\epsilon>0$. Como no Teorema 3.3.3, é possível obter um aberto $U \subseteq \bar{D} \operatorname{com} V \subseteq U$ e $|u(t)|<\epsilon, \forall t \in U$.

Para cada $z \in \bar{D} \backslash U$ temos que $u(z) \neq 0$ e consequentemente pelo Teorema 3.3.3 temos que $|\varphi(z)|<1$. Logo $\varphi(\bar{D} \backslash U) \subseteq D$. Como $\bar{D} \backslash U$ é compacto e $\varphi$ é contínua em $\bar{D}$, segue que $\varphi(\bar{D} \backslash U)$ é um compacto de $D$. Portanto, pelo Teorema 3.4.6 (Denjoy-Wolff), temos que $\varphi^{n}$ converge uniformemente para $z_{0} \operatorname{em} \varphi(\bar{D} \backslash U)$.

Como $u\left(z_{0}\right)=0$, temos que $z_{0} \in U$, que é aberto. Portanto existe $\delta>0$ tal que $D_{\delta}\left(z_{0}\right) \subseteq U$. Como $\varphi^{n} \longrightarrow z_{0}$ uniformemente em $\varphi(\bar{D} \backslash U), \exists N_{1} \in \mathbb{N}$ tal que $\mid \varphi^{n}(z)-$ $z_{0} \mid<\delta, \forall n \geq N_{1}$ e $\forall z \in \varphi(\bar{D} \backslash U)$. Portanto, sem perda de generalidade temos que $\varphi^{n}(\bar{D} \backslash U) \subseteq U$, para $n \geq N_{1}$.

Agora, se $z \in U$, afirmamos que existe $N_{2}$ tal que se $n \geq N_{2}$ então $\varphi^{n}(z) \in U$. Vamos supor por absurdo que tal afirmação é falsa, ou seja, que para todo $n \in \mathbb{N}$ existe $k_{n} \geq n$ 
tal que $\varphi^{k_{n}}(z) \notin U$. Desta forma, $\varphi^{m}\left(\varphi^{k_{n}}(z)\right)=\varphi^{m+k_{n}}(z) \in U$, para todo $m \geq N_{1}$. Assim, fixado $n \in \mathbb{N}$, para o natural $N_{1}+k_{n}$ existe $k_{n^{\prime}} \geq N_{1}+k_{n}$ tal que $\varphi^{k_{n^{\prime}}}(z) \notin U$, o que é uma contradição.

Desta forma, tomando $N=\max \left\{N_{1}, N_{2}\right\}$ teremos

$$
\left|u\left(\varphi^{n}(z)\right)\right|<\epsilon, \forall n \geq N \text { e } \forall z \in \bar{D} \text {. }
$$

Agora sejam $n>N+1$ e $f \in \mathcal{A}(D)$, pelo Lema 3.3.2 e por (3.9) temos que

$$
\sup _{z \in \bar{D}}\left|\left(\left(u C_{\varphi}\right)^{n}(f)\right)(z)\right|=\sup _{z \in \bar{D}}\left|u(z) u(\varphi(z)) \cdots u\left(\varphi^{n-1}(z)\right) f\left(\varphi^{n}(z)\right)\right|<\|u\|^{N} \epsilon^{n-N}\|f\| .
$$

Portanto $\left\|\left(u C_{\varphi}\right)^{n}\right\| \leq\|u\|^{N} \epsilon^{n-N}$ e assim $\left\|\left(u C_{\varphi}\right)^{n}\right\|^{\frac{1}{n}} \leq\|u\|^{\frac{N}{n}} \epsilon^{\frac{n-N}{n}}$. Desta forma

$$
\lim _{n \rightarrow \infty}\left\|\left(u C_{\varphi}\right)^{n}\right\|^{\frac{1}{n}} \leq \lim _{n \rightarrow \infty}\|u\|^{\frac{N}{n}} \epsilon^{\frac{n-N}{n}} \longrightarrow \epsilon \text {, ou seja, }
$$

$\lim _{n \rightarrow \infty}\left\|\left(u C_{\varphi}\right)^{n}\right\|^{\frac{1}{n}} \leq \epsilon, \forall \epsilon>0$, isto é,

$$
\lim _{n \rightarrow \infty}\left\|\left(u C_{\varphi}\right)^{n}\right\|^{\frac{1}{n}}=0
$$

como queríamos.

(3) Se $z_{0}=0$ temos pelo Corolário 3.4 .4 que $\sigma\left(u C_{\varphi}\right)=\left\{u\left(z_{0}\right) \varphi^{\prime}\left(z_{0}\right)^{n}: n \in \mathbb{N}, n \geq\right.$ $1\} \cup\left\{0, u\left(z_{0}\right)\right\}$. Se $z_{0} \neq 0$, considere o automorfismo $p: \bar{D} \longrightarrow \bar{D}$ dado por $p(z)=\frac{z_{0}-z}{1-\overline{z_{0}} z}=$ $(-1) \varphi_{z_{0}}(z)$. É claro que $p \in \mathcal{A}(D)$ e que $p \circ p=i d$.

Vamos definir $\psi=p \circ \varphi \circ p$ e $u^{*}=u \circ p$. Assim $\psi, u^{*} \in \mathcal{A}(D)$ e $\psi(\bar{D}) \subseteq \bar{D}$. Afirmamos que $u^{*} C_{\psi}$ é compacto.

Com efeito, se $u^{*}(z) \neq 0$ então $u(p(z)) \neq 0$. Como $u C_{\varphi}$ é compacto, o Teorema 3.3.3 garante que $|\varphi(p(z))|<1$. Agora, pela Proposição 1.2.14 temos que $p(D)=D$, o que garante que $|p(\varphi(p(z)))|<1$, ou seja, $|\psi(z)|<1$. Pelo Teorema 3.3.3, concluímos que $u^{*} C_{\psi}$ é compacto.

Considere o operador de composição $C_{p}$. Pela Proposição 3.2 .3 temos que $C_{p}$ é invertível e $C_{p}^{-1}=C_{p^{-1}}=C_{p}$. Também vale que $C_{p} \circ\left(u^{*} C_{\psi}\right) \circ C_{p}^{-1}=u C_{\varphi}$. De fato, se $f \in \mathcal{A}(D)$ então

$$
\left(C_{p} \circ\left(u^{*} C_{\psi}\right) \circ C_{p}^{-1}\right)(f)=\left(C_{p} \circ\left(u^{*} C_{\psi}\right) \circ C_{p}\right)(f)=\left(C_{p} \circ\left(u^{*} C_{\psi}\right)\right)(f \circ p)=
$$




$$
=C_{p}\left(u^{*} \cdot f \circ p \circ \psi\right)=\left(u^{*} \circ p\right) \cdot(f \circ p \circ \psi \circ p)=u \cdot(f \circ \varphi)=u C_{\varphi}(f) .
$$

Como, pela Proposição 2.1.12, $\sigma\left(C_{p} \circ\left(u^{*} C_{\psi}\right) \circ C_{p}^{-1}\right)=\sigma\left(u^{*} C_{\psi}\right)$ segue que $\sigma\left(u C_{\varphi}\right)=$ $\sigma\left(C_{p} \circ\left(u^{*} C_{\psi}\right) \circ C_{p}\right)=\sigma\left(u^{*} C_{\psi}\right)$.

Agora $\psi(0)=p(\varphi(p(0)))=p\left(\varphi\left(z_{0}\right)\right)=p\left(z_{0}\right)=0$. Então, pelo Corolário 3.4.4,

$$
\sigma\left(u^{*} C_{\psi}\right)=\left\{u^{*}(0) \psi^{\prime}(0)^{n}: n \in \mathbb{N}, n \geq 1\right\} \cup\left\{0, u^{\star}(0)\right\}
$$

Como $u^{*}(0)=u(p(0))=u\left(z_{0}\right)$ e $\psi^{\prime}(0)=\varphi^{\prime}\left(z_{0}\right)$, temos que

$$
\sigma\left(u C_{\varphi}\right)=\left\{u\left(z_{0}\right) \varphi^{\prime}\left(z_{0}\right)^{n}: n \in \mathbb{N}, n \geq 1\right\} \cup\left\{0, u\left(z_{0}\right)\right\},
$$

concluindo a demonstração do teorema. 


\section{Capítulo 4}

\section{A álgebra $\mathcal{H}^{\infty}(D)$}

Os resultados que serào apresentados neste capítulo fazem parte dos trabalhos apresentados por R. Aron, M. Lindström e P. Galindo em [1], M. Lindström e P. Galindo em [10] e A. Ülger em [34].

Na primeira seção deste capítulo estudaremos o espectro de $\mathcal{H}^{\infty}(D)$, bem como as partes de Gleason de elementos de $\mathcal{M}\left(\mathcal{H}^{\infty}(D)\right)$. Veremos que no caso da álgebra $\mathcal{H}^{\infty}(D)$, existem homomorfismos $\phi: \mathcal{H}^{\infty}(D) \longrightarrow \mathbb{C}$ que não podem ser caracterizados como uma avaliação.

Depois daremos uma caracterização para que um homomorfismo $T: \mathcal{H}^{\infty}(D) \longrightarrow$ $\mathcal{H}^{\infty}(D)$ seja um operador de composição, bem como caracterizaremos a compacidade de tais operadores.

Por fim mostraremos que todo homomorfismo $T: \mathcal{H}^{\infty}(D) \longrightarrow \mathcal{H}^{\infty}(D)$ w-compacto é compacto.

\subsection{Partes de Gleason de $\mathcal{M}\left(\mathcal{H}^{\infty}(D)\right)$}

Para cada $z \in D$, a avaliação $\delta_{z}$ é um elemento de $\mathcal{M}\left(\mathcal{H}^{\infty}(D)\right)$. No entanto, existem elementos de $\mathcal{M}\left(\mathcal{H}^{\infty}(D)\right)$ que não são avaliações, como mostra o seguinte exemplo. 
Exemplo 4.1.1 Considere o conjunto

$$
I=\left\{f \in \mathcal{H}^{\infty}(D): f\left(x_{n}\right) \rightarrow 0, \text { para toda sequência }\left(x_{n}\right) \subseteq\right] 0,1\left[\text { tal que } x_{n} \rightarrow 1\right\} .
$$

É claro que $I$ é um ideal próprio de $\mathcal{H}^{\infty}(D)$ e, consequentemente, está contido em algum ideal maximal $M$. Pelo Teorema 2.1.19, existe um homomorfismo não nulo $\phi: \mathcal{H}^{\infty}(D) \longrightarrow$ $\mathbb{C}$ tal que $\operatorname{ker}(\phi)=M$, ou seja, $\phi(f)=0, \forall f \in M$. Agora, se $f(z)=z-1$, é claro que $f \in I$ e $\delta_{z}(f) \neq 0, \forall z \in D$.

Por outro lado, existe uma aplicação natural contínua $\pi: \mathcal{M}\left(\mathcal{H}^{\infty}(D)\right) \longrightarrow \bar{D}$ definida por $\pi(\phi)=\phi(i d)=\widehat{i d}(\phi), \forall \phi \in \mathcal{M}\left(\mathcal{H}^{\infty}(D)\right)$.

A proposição a seguir descreve as propriedades da aplicação $\pi$.

Proposição 4.1.2 A aplicação $\pi$ definida acima é sobrejetora e, quanto restrita a $\delta(D)$ é injetora. Além disso, $\pi^{-1}$ leva $D$ homeomorficamente sobre $\delta(D)$.

Demonstração: Para cada $\delta_{z} \in \delta(D)$, temos que $z=\pi\left(\delta_{z}\right)$. Logo $D \subseteq \pi\left(\mathcal{M}\left(\mathcal{H}^{\infty}(D)\right)\right) \subseteq$ $\bar{D}$. Como $\mathcal{M}\left(\mathcal{H}^{\infty}(D)\right)$ é compacto, segue que $\pi\left(\mathcal{M}\left(\mathcal{H}^{\infty}(D)\right)\right)=\bar{D}$, o que prova que $\pi$ é sobrejetora. Segue facilmente da definição de $\pi$ que $\pi$ restrita a $\delta(D)$ é injetora.

Resta provar que $\pi^{-1}$ leva $D$ homeomorficamente sobre $\delta(D)$.

Se $\phi \in \pi^{-1}(D)$, então $\pi(\phi)=\beta$, para algum $\beta \in D$. Se $f \in \mathcal{H}^{\infty}(D)$ é tal que $f(\beta)=0$, pela Proposição 1.2 .4 existem $g \in \mathcal{H}(D)$ e $n \in \mathbb{N}$ tais que $f(z)=(z-\beta)^{n} g(z)$, $\forall z \in D$. Assim $\phi(f)=(\phi(i d-\beta 1))^{n} \cdot \phi(g)=0=f(\beta)$, ou seja, $\phi(f)=\delta_{\beta}(f)$.

Agora, se $f(\beta)=\alpha \neq 0$, considerando $h=f-\alpha \mathbf{1}$, obtemos $0=\phi(h)=\phi(f-\alpha \mathbf{1})$. $\operatorname{Logo} \phi(f)=\alpha=\delta_{\beta}(f)$.

Então provamos que $\pi^{-1}(z)=\delta(z)=\delta_{z} \cdot \forall z \in D$, ou seja, $\left.\pi^{-1}\right|_{D}=\left.\delta\right|_{D}$, e pelo Teorema 2.2 .5 a aplicação $\delta$ é contínua. Assim $\left.\pi^{-1}\right|_{D}$ é contínua, e como $\pi$ é contínua e $\pi$ restrita a $\delta(D)$ é bijetora, segue o resultado.

Se $a \in \mathbb{C}$ é tal que $|a|=1$, denotaremos por $\mathcal{M}_{a}=\left\{\phi \in \mathcal{M}\left(\mathcal{H}^{\infty}(D)\right): \pi(\phi)=a\right\}$. Como $\pi$ é contínua, é claro que $\mathcal{M}_{a}$ é um subconjunto compacto de $\mathcal{M}\left(\mathcal{H}^{\infty}(D)\right)$. 
Os conjuntos da forma $\mathcal{M}_{a}$ consistem dos homomorfismos de $\mathcal{M}\left(\mathcal{H}^{\infty}(D)\right)$ que podem ser de certa forma identificados como "avaliações em a", como mostra a seguinte proposição.

Proposição 4.1.3 Sejam $f \in \mathcal{H}^{\infty}(D)$ e $a \in \mathbb{C}$ tal que $|a|=1$. Seja $\left(a_{n}\right) \subseteq D$ tal que $a_{n} \rightarrow a$ e suponha que $\lim _{n \rightarrow \infty} f\left(a_{n}\right)=\beta$ existe. Então existe $\phi \in \mathcal{M}_{a}$ tal que $\phi(f)=\beta$.

Demonstração: Seja $J$ a coleção de todas as funções $g \in \mathcal{H}^{\infty}(D)$ tais que $\lim _{n \rightarrow \infty} g\left(a_{n}\right)=0$. É fácil ver que $J \neq \emptyset$ e que $J$ é um ideal próprio de $\mathcal{H}^{\infty}(D)$.

Portanto, pelo Teorema 2.1 .19 existe $\phi \in \mathcal{M}\left(\mathcal{H}^{\infty}(D)\right)$ tal que $\phi(g)=0, \forall g \in J$. Agora, as funções $i d-a 1$ e $f-\beta 1$ pertencem a $J$. Então $\phi(i d)=a$, ou seja, $\phi \in \mathcal{M}_{a}$; e $o(f)=\beta$. Logo segue o resultado.

Um dos principais resultados relativos ao espectro de $\mathcal{H}^{\infty}(D)$ é conhecido como o Teorema da corona, e afirma que $\delta(D)$ é $w^{*}$-denso em $\mathcal{M}\left(\mathcal{H}^{\infty}(D)\right)$. Este teorema foi provado por L. Carleson em [4].

É claro que para cada $z \in D$ temos que $P\left(\delta_{z}\right)=\delta(D)$. Desta forma estaremos interessados nas partes de Gleason de $\mathcal{M}\left(\mathcal{H}^{\infty}(D)\right)$ que não estão contidas em $\delta(D)$.

I. J. Schark exibiu em [32] pontos $m \in \mathcal{M}\left(\mathcal{H}^{\infty}(D)\right) \backslash \delta(D)$ tais que $P(m)$ não é trivial. Em [17], K. Hoffman dá exemplos de elementos de $\mathcal{M}\left(\mathcal{H}^{\infty}(D)\right) \backslash \delta(D)$ que possuem partes de Gleason triviais.

O seguinte teorema apresenta uma relação entre partes de Gleason de elementos de $\mathcal{M}\left(\mathcal{H}^{\infty}(D)\right) \backslash \delta(D)$ e os conjuntos da forma $\mathcal{M}_{a}$.

Proposição 4.1.4 Se $m \in \mathcal{M}\left(\mathcal{H}^{\infty}(D)\right) \backslash \delta(D)$, então existe a $\in \mathbb{C}$ tal que $|a|=1$ e $P(m) \subseteq \mathcal{M}_{a}$.

Demonstração: Seja $a=\pi(m)=\widehat{i d}(m)$. Como pela Proposição $4.1 .2 \pi$ restrita a $\delta(D)$ é bijetora, temos que $|a|=1$. Agora, se $f: D \longrightarrow \mathbb{C}$ é dada por $f(z)=\frac{z}{a}, \forall z \in D$, então temos que $f \in \mathcal{H}^{\infty}(D)$ e $\|\hat{f}\|=1$. Como $\hat{f}(m)=1$, o Lema 2.3 .3 garante que $\hat{f}(n)=1$, para todo $n \in P(m)$. Ou seja, $a=n(i d)=\pi(n), \forall n \in P(m)$. Portanto $P(m) \subseteq \mathcal{M}_{a}$, como queríamos demonstrar. 
Para calcular as partes de Gleason de elementos de $\mathcal{M}\left(\mathcal{H}^{\infty}(D)\right)$, iremos procurar por aplicações que coordenam pontos de $\mathcal{M}\left(\mathcal{H}^{\infty}(D)\right)$ em discos analíticos de $\mathcal{M}\left(\mathcal{H}^{\infty}(D)\right)$. Assim enunciaremos sem demonstrar o teorema que realiza tal associação. Sua demonstração foi feita por K. Hoffman em [16].

No próximo teorema e na próxima seção, se $M$ é um subconjunto de $\mathcal{M}\left(\mathcal{H}^{\infty}(D)\right)$, a notação $(M,\|\cdot\|)$ estará significando que em $M$ estamos considerando a norma em $\mathcal{H}^{\infty}(D)^{\prime}$.

Teorema 4.1.5 Seja $m \in \mathcal{M}\left(\mathcal{H}^{\infty}(D)\right)$. Então existe uma aplicação $L_{m}: D \longrightarrow P(m)$ tal que se $P(m)$ não é trivial, então $\hat{f} \circ L_{m} \in \mathcal{H}^{\infty}(D), \forall f \in \mathcal{H}^{\infty}(D)$ e $L_{m}(0)=m$. Além disso, $L_{m}$ é um homeomorfismo sobre $(P(m),\|\cdot\|)$ e também sobre $(P(m), w)$.

Observação 4.1.6 Se $\alpha \in D$, sabemos que $P\left(\delta_{\alpha}\right)=\delta(D)$. Neste caso é fácil construir a aplicação $L_{\alpha}$ do teorema acima. Com efeito, consideremos a aplicação $L_{\alpha}: D \longrightarrow \delta(D)$ dada por $L_{\alpha}(z)=\delta_{\frac{z+\alpha}{1+\bar{\alpha} z}}$, isto é, $L_{\alpha}(z)=\delta_{\varphi_{-\alpha}(z)}$, onde $\varphi_{-\alpha}$ foi definida no Capítulo 1 .

Se $f \in \mathcal{H}^{\infty}(D)$. então $\left(\hat{f} \circ L_{\alpha}\right)(z)=f\left(\frac{z+\alpha}{1+\bar{\alpha} z}\right)=\left(f \circ \varphi_{-\alpha}\right)(z), \forall z \in D$. Logo $\hat{f} \circ L_{\alpha} \in \mathcal{H}^{\infty}(D), \forall f \in \mathcal{H}^{\infty}(D)$.

Não é difícil ver que $L_{\alpha}^{-1}: \delta(D) \longrightarrow D$ é dada por $L_{\alpha}^{-1}\left(\delta_{z}\right)=\varphi_{\alpha}(z)$. Também não é difícil ver que $L_{\alpha}$ é um homeomorfismo sobre $(\delta(D),\|\cdot\|)$ e também sobre $(\delta(D), w)$.

Caracterizar as partes de Gleason de elementos de $\mathcal{M}\left(\mathcal{H}^{\infty}(D)\right)$ da forma como foi feito no Teorema 4.1.5 é útil para provar que todo homomorfismo $w$-compacto de $\mathcal{H}^{\infty}(D)$ em $\mathcal{H}^{\infty}(D)$ é compacto. Tal resultado será provado na seção 3 deste capítulo.

\subsection{Operadores de Composição de $\mathcal{H}^{\infty}(D)$ em $\mathcal{H}^{\infty}(D)$}

Dizemos que $T: \mathcal{H}^{\infty}(D) \longrightarrow \mathcal{H}^{\infty}(D)$ é um operador de composição se existe $\varphi \in$ $\mathcal{H}^{\infty}(D)$ tal que $\varphi(D) \subseteq D$ e $T(f)=f \circ \varphi, \forall f \in \mathcal{H}^{\infty}(D)$. Denotaremos um operador de composição por $C_{\varphi}$. É fácil ver que todo operador de composição de $\mathcal{H}^{\infty}(D)$ em $\mathcal{H}^{\infty}(D)$ é um homomorfismo contínuo. 
Nesta seção iremos apresentar uma caracterização para que um homomorfismo de $\mathcal{H}^{\infty}(D)$ em $\mathcal{H}^{\infty}(D)$ seja um operador de composição. Em seguida, daremos uma caracterização para a compacidade e $w$-compacidade de tais operadores.

A próxima proposição nos mostra que todo homomorfismo de $\mathcal{H}^{\infty}(D)$ em $\mathcal{H}^{\infty}(D)$ é contínuo.

Proposição 4.2.1 Sejam $T: \mathcal{H}^{\infty}(D) \longrightarrow \mathcal{H}^{\infty}(D)$ um homomorfismo e $f \in \mathcal{H}^{\infty}(D)$ tal que $\|f\| \leq 1$. Então $\|T(f)\| \leq 1$.

Demonstração: Seja $f \in \mathcal{H}^{\infty}(D)$ tal que $\|f\| \leq 1$. Queremos provar que

$$
\sup _{z \in D}|T(f)(z)| \leq 1 \text {. }
$$

Para cada $z \in D$, a aplicação $\delta_{z} \circ T: \mathcal{H}^{\infty}(D) \longrightarrow \mathbb{C}$ é um homomorfismo complexo não nulo e portanto, pelo Teorema 2.1.6 temos que $\left\|\delta_{z} \circ T\right\|=1$. Logo $\left|\left(\delta_{z} \circ T\right)(f)\right| \leq 1$, ou seja,

$$
|T(f)(z)| \leq 1, \forall z \in D
$$

como queríamos.

Observação 4.2.2 Vale ressaltar que a proposição acima vale para qualquer homomorfismo $T: A \longrightarrow A$, onde $A$ é uma álgebra uniforme.

Proposição 4.2.3 Seja $T: \mathcal{H}^{\infty}(D) \longrightarrow \mathcal{H}^{\infty}(D)$ um homomorfismo. Então $T$ é um operador de composição se, e somente se, $T(i d)$ não é constante ou é constante de valor absoluto menor que 1 .

Demonstração: Vamos supor inicialmente que $T(i d)$ não é constante ou é constante de valor absoluto menor que 1. Seja $\varphi=T(i d)$. Segue da Proposição 4.2.1 que $\|\varphi\| \leq 1$. Assim $\varphi(D) \subseteq \bar{D}$. Agora, se $\varphi$ não é constante, o Teorema 1.2.7 (Aplicação Aberta), nos diz que $\varphi(D)$ é aberto e portanto $\varphi(D) \subseteq D$, o que implica que $\varphi: D \rightarrow D$. Se $T(i d)$ for constante de valor absoluto menor que 1 , é imediato que $\varphi: D \rightarrow D$.

Agora considere $f \in \mathcal{H}^{\infty}(D)$ e $z_{0} \in D$, assim segue que $\left|\varphi\left(z_{0}\right)\right|<1$. Defina $g: D \longrightarrow$ $\mathbb{C}$ por

$$
g(z)=\left\{\begin{array}{ll}
\frac{f(z)-f\left(\varphi\left(z_{0}\right)\right)}{z-\varphi\left(z_{0}\right)} & z \neq \varphi\left(z_{0}\right) \\
f^{\prime}\left(\varphi\left(z_{0}\right)\right) & z=\varphi\left(z_{0}\right)
\end{array} .\right.
$$


Afirmamos que $g \in \mathcal{H}^{\infty}(D)$. De fato, $g \in \mathcal{C}(D) \cap \mathcal{H}\left(D \backslash\left\{\varphi\left(z_{0}\right)\right\}\right)$. Então, pelo Corolário 1.2.27, temos que $g \in \mathcal{H}(D)$. Agora vamos provar que $g$ é limitada em $D$. Com efeito, dado $\epsilon=1, \exists r>0$ tal que se $z \in D$ e $\left|z-\varphi\left(z_{0}\right)\right|<r$ entào $|g(z)|<1+\left|f^{\prime}\left(\varphi\left(z_{0}\right)\right)\right|$.

Se $z \in D$ e $\left|z-\varphi\left(z_{0}\right)\right| \geq r$ temos que $|g(z)| \leq \frac{2 M}{r}$, onde $M$ é tal que $|f(z)| \leq M, \forall z \in$ D.

Portanto, tomando $K=\max \left\{1+\left|f^{\prime}\left(\varphi\left(z_{0}\right)\right)\right|, \frac{2 M}{r}\right\}$, teremos que $|g(z)| \leq K, \forall z \in D$, provando que $g$ é limitada em $D$.

Como $g=\frac{f-f\left(\varphi\left(z_{0}\right)\right)}{i d-\varphi\left(z_{0}\right)}$ em $D \backslash\left\{\varphi\left(z_{0}\right)\right\}$, temos que

$$
T(g)=\frac{T(f)-f\left(\varphi\left(z_{0}\right)\right)}{T(i d)-\varphi\left(z_{0}\right)}=\frac{T(f)-f\left(\varphi\left(z_{0}\right)\right)}{\varphi-\varphi\left(z_{0}\right)} .
$$

Logo $T(f)-f\left(\varphi\left(z_{0}\right)\right)=T(g)\left(\varphi-\varphi\left(z_{0}\right)\right)$ em $D \backslash\left\{\varphi\left(z_{0}\right)\right\}$, que é aberto. Como $T(f)-$ $f\left(\varphi\left(z_{0}\right)\right)$ e $T(g)\left(\varphi-\varphi\left(z_{0}\right)\right)$ são holomorfas em $D$, segue do Corolário 1.2.3, (Princípio do Prolongamento Analítico), que $T(f)-f\left(\varphi\left(z_{0}\right)\right)=T(g)\left(\varphi-\varphi\left(z_{0}\right)\right)$ em $D$ e portanto

$$
\begin{gathered}
T(f)\left(z_{0}\right)-f\left(\varphi\left(z_{0}\right)\right)=T(g)\left(\varphi\left(z_{0}\right)-\varphi\left(z_{0}\right)\right)=0, \\
\text { ou seja, } T(f)\left(z_{0}\right)=f\left(\varphi\left(z_{0}\right)\right) .
\end{gathered}
$$

Como $z_{0}$ era arbitrário. temos que $T(f)=f \circ \varphi$, ou seja, $T$ é um operador de composição.

Reciprocamente, se $T(f)=f \circ \varphi$, então $\varphi=T(i d)$. Vamos supor que $T(i d)$ seja constante com $\|T(i d)\|=1$. Assim $\exists a$ tal que $\varphi(z)=a, \forall z \in D$; e $|a|=1$, uma vez que $\|\varphi\|=1$. Desta forma $\varphi(D)$ não está contido em $D$, uma contradição com a hipótese de $T$ ser um operador de composição.

A próxima proposição é um resultado análogo à Proposição 3.2 .4 , porém para a álgebra $\mathcal{H}^{\infty}(D)$.

Proposição 4.2.4 Considere $C_{\varphi}: \mathcal{H}^{\infty}(D) \longrightarrow \mathcal{H}^{\infty}(D)$ um operador de composição. As seguintes afirmações são equivalentes:

1. $C_{\varphi}$ é compacto;

2. $C_{\varphi}$ é fracamente compacto;

3. $\varphi(D) \varsubsetneqq D$. 
Demonstração: A demonstração desta proposição é similar à feita para $\mathcal{A}(D)$ no Capítulo 3. As diferenças entre elas dão-se sobretudo por causa do item (3).

$(1) \Rightarrow(2)$ Segue imediatamente da Proposição 1.2.57.

(2) $\Rightarrow$ (3) Vamos supor por absurdo que $\varphi(D)=D$. Para cada $j \in \mathbb{N}$ existe $w_{j} \in D$ tal que $1-\frac{1}{j}<\left|w_{j}\right|<1$. Como $\varphi(D)=D$, existe $z_{j}$ tal que $w_{j}=\varphi\left(z_{j}\right)$. Assim temos que $1-\frac{1}{j}<\left|\varphi\left(z_{j}\right)\right|<1$. Portanto $\exists z_{0}$ tal que $\varphi\left(z_{j}\right) \longrightarrow z_{0}$, com $\left|z_{0}\right|=1$.

Procedendo de modo análogo à demonstração da Proposição 3.2.4, a partir da sequência $\left(\varphi\left(z_{j}\right)\right)_{j \in \mathbb{N}}$ e do número complexo $z_{0}$, obtemos $u \in \mathcal{H}^{\infty}(D)^{\prime}, f \in \mathcal{H}^{\infty}(D)$ e uma sequência $\left(l^{n}\right) \subseteq \mathcal{H}^{\infty}(D)$ tais que $u\left(l^{n} \circ \varphi\right)=1, \forall n \in \mathbb{N}, u(f)=0$ e $u\left(l^{n} \circ \varphi\right) \longrightarrow u(f)$, o que nos leva a uma contradição.

$(3) \Rightarrow$ (1) Como $\varphi(D) \varsubsetneqq D$, temos que $\exists 0<r<1$ tal que $\varphi(D) \subseteq D_{r}(0)$. Então $\overline{\varphi(D)} \subseteq \overline{D_{r}(0)} \subseteq D$ e consequentemente $\overline{\varphi(D)}$ é um compacto de $D$.

Se $C_{\varphi}$ não é compacto, então existem $\epsilon>0$ e uma sequência $\left(f_{n}\right) \subseteq \mathcal{H}^{\infty}(D)$ com $\left\|f_{n}\right\| \leq 1$ e $\left\|C_{\varphi}\left(f_{n}\right)-C_{\varphi}\left(f_{m}\right)\right\| \geq \epsilon, \forall n \neq m$. Como $\left\{f_{n}: n \in \mathbb{N}\right\}$ é uma família limitada, segue do Teorema 1.2.22 (Montel) que $\left\{f_{n}: n \in \mathbb{N}\right\}$ é uma família normal, e portanto existe uma subsequência $\left(f_{n_{k}}\right) \subseteq\left(f_{n}\right)$ convergindo sobre os compactos de D. Se $k_{0} \neq k$ :

$$
\begin{gathered}
\sup _{w \in \overline{\varphi(D)}}\left|f_{n_{k_{0}}}(w)-f_{n_{k}}(w)\right| \geq \sup _{w \in \varphi(D)}\left|f_{n_{k_{0}}}(w)-f_{n_{k}}(w)\right|= \\
=\sup _{z \in D}\left|f_{n_{k_{0}}}(\varphi(z))-f_{n_{k}}(\varphi(z))\right|=\left\|C_{\varphi}\left(f_{n_{k_{0}}}\right)-C_{\varphi}\left(f_{n_{k}}\right)\right\| \geq \epsilon .
\end{gathered}
$$

Mas isto é uma contradição, uma vez que $\left(f_{n_{k}}\right)$ é uniformemente convergente sobre os compactos de $D$, já que $\overline{\varphi(D)}$ é um compacto de $D$. Logo $C_{\varphi}$ é compacto, como queríamos.

\subsection{Homomorfismos $w$-Compactos de $\mathcal{H}^{\infty}(D)$ em $\mathcal{H}^{\infty}(D)$.}

Nesta seção iremos demonstrar que todo homomorfismo w-compacto $T: \mathcal{H}^{\infty}(D) \longrightarrow$ $\mathcal{H}^{\infty}(D)$ é compacto.

Seja $T: \mathcal{H}^{\infty}(D) \longrightarrow \mathcal{H}^{\infty}(D)$ um homomorfismo. Definimos a aplicação $\phi_{T}$ : 
$\left(\mathcal{H}\left(\mathcal{H}^{\infty}(D)\right), w^{*}\right) \longrightarrow\left(\mathcal{M}\left(\mathcal{H}^{\infty}(D)\right), w^{*}\right)$ da seguinte maneira:

$$
\phi_{T}(n)=n \circ T \text {, para todo } n \in \mathcal{M}\left(\mathcal{H}^{\infty}(D)\right) .
$$

Observamos que $\phi_{T}=\left.T^{\star}\right|_{\mathcal{M}\left(\mathcal{H}^{\propto}(D)\right.}$, portanto pelo Teorema 1.2.39, temos que $\phi_{T}$ é contínua.

Vamos provar que $\phi_{T}\left(\mathcal{M}\left(\mathcal{H}^{\infty}(D)\right)\right)$ é $w$-fechado. Pelo Teorema 2.1.20 Temos que $\mathcal{U}\left(\mathcal{H}^{\infty}(D)\right)$ é $w^{*}$-compacto. Como $\phi_{T}$ é $w^{*}$-contínua, segue que $\phi_{T}\left(\mathcal{M}\left(\mathcal{H}^{\infty}(D)\right)\right)$ é $w^{*}$ compacto, e portanto $w^{*}$-fechado. Pela Observação 1.2 .35 , segue que $\phi_{T}\left(\mathcal{M}\left(\mathcal{H}^{\infty}(D)\right)\right)$ é $u$-fechado, como queríamos.

Vamos agora mostrar que existe $m_{0} \in \mathcal{M}\left(\mathcal{H}^{\infty}(D)\right)$ tal que $\phi_{T}(\delta(D)) \subseteq P\left(m_{0}\right)$. Para tal, sejam $f \in \mathcal{H}^{\infty}(D)$ e $g=T(f)$. Afirmamos que $\left.\hat{f} \circ \phi_{T} \circ \delta\right|_{D}: D \longrightarrow \mathbb{C}$ é analítica em $D$. Com efeito, se $z \in D$ então

$$
\hat{f} \circ \phi_{T} \circ \delta(z)=\hat{f} \circ \phi_{T}\left(\delta_{z}\right)=\hat{f}\left(\delta_{z} \circ T\right)=\delta_{z}(T(f))=\delta_{z}(g)=g(z) .
$$

Logo $\hat{f} \circ \phi_{T} \circ \delta$ é analítica em $D, \forall f \in \mathcal{H}^{\infty}(D)$. Como $D$ é conexo, pelo Teorema 2.3.8 existe $m_{0} \in \mathcal{M}\left(\mathcal{H}^{\infty}(D)\right)$ tal que $\phi_{T} \circ \delta(D)=\phi_{T}(\delta(D)) \subseteq P\left(m_{0}\right)$.

Se $P\left(m_{0}\right)$ é trivial, isto é, se $P\left(m_{0}\right)=\left\{m_{0}\right\}$, para cada $z \in D$ temos que $\phi_{T}\left(\delta_{z}\right)=m_{0}$, ou seja, $\delta_{z} \circ T=m_{0}$. Portanto, para cada $f \in \mathcal{H}^{\infty}(D)$ temos que $\left(\delta_{z} \circ T\right)(f)=m_{0}(f)$, isto é, $T(f)(z)=m_{0}(f)$.

Logo $T(f)$ é uma função constante e consequentemente $\operatorname{dim}(\operatorname{im}(T))=1$. Pelo Teorema 1.2.48 segue que $T$ é compacto.

O objetivo desta seção é mostrar que todo homomorfismo $T: \mathcal{H}^{\infty}(D) \longrightarrow \mathcal{H}^{\infty}(D)$ $w$-compacto é compacto. Acabamos de mostrar que se $P\left(m_{0}\right)$ é trivial, qualquer homomorfismo $T: \mathcal{H}^{\infty}(D) \longrightarrow \mathcal{H}^{\infty}(D)$ é compacto. Desta forma, no que se segue iremos supor que $P\left(m_{0}\right)$ não é trivial. Iremos provar um Lema e uma Proposição e com o auxílio destes provar o principal resultado da seção.

Lema 4.3.1 Seja $T: \mathcal{H}^{\infty}(D) \longrightarrow \mathcal{H}^{\infty}(D)$ um homomorfismo w-compacto. Então existe $m_{0} \in \mathcal{M}\left(\mathcal{H}^{\infty}(D)\right)$ tal que $\phi_{T}\left(\mathcal{M}\left(\mathcal{H}^{\infty}(D)\right)\right) \subseteq P\left(m_{0}\right)$.

Demonstração: Pelo feito no início desta seção, temos que existe $m_{0} \in \mathcal{M}\left(\mathcal{H}^{\infty}(D)\right)$ tal que $\phi_{T}(\delta(D)) \subseteq P\left(m_{0}\right)$. Tomemos $n_{0} \in \mathcal{M}\left(\mathcal{H}^{\infty}(D)\right) \backslash \delta(D)$ e vamos supor por absurdo 
que $\phi_{T}\left(n_{0}\right) \notin P\left(m_{0}\right)$. Pelo Lema 2.3 .3 existe uma sequência $\left(f_{j}\right) \subseteq \mathcal{H}^{\infty}(D)$ com $\left\|f_{j}\right\| \leq 1$, $\forall j \in \mathbb{N},\left|\widehat{f}_{j}\left(\phi_{T}\left(\delta_{\lambda}\right)\right)\right| \longrightarrow 1, \forall \lambda \in D$ e $\left|\widehat{f}_{j}\left(\phi_{T}\left(n_{0}\right)\right)\right| \nrightarrow 1$. Entào existe uma subsequência $\left(f_{j_{k}}\right) \subseteq\left(f_{j}\right) \operatorname{com}\left|\widehat{j_{j_{k}}}\left(o_{T}\left(n_{0}\right)\right)\right| \leq \beta<1$, para algum $\beta$ e para todo $k$.

Como $T$ é $w$-compacto. sem perda de generalidade, existe $f \in \mathcal{H}^{\infty}(D)$ tal que $\left(T\left(f_{j_{k}}\right)\right)$ converge fracamente para $f$. Em particular, para todo $n \in \mathcal{M}\left(\mathcal{H}^{\infty}(D)\right) \subseteq \mathcal{H}^{\infty}(D)^{\prime}$, temos pela Observação 1.2 .32 que $n\left(T\left(f_{j_{k}}\right)\right) \longrightarrow n(f)$, ou seja, $\widehat{f_{j_{k}}}(n \circ T) \longrightarrow n(f)$. Logo $\widehat{f_{j_{k}}}\left(\phi_{T}(n)\right) \longrightarrow n(f), \forall n \in \mathcal{M}\left(\mathcal{H}^{\infty}(D)\right)$. E portanto

$$
\left|\widehat{f_{j_{k}}}\left(o_{T}(n)\right)\right| \longrightarrow|n(f)|, \forall n \in \mathcal{M}\left(\mathcal{H}^{\infty}(D)\right), k \rightarrow \infty
$$

Por outro lado, através do Teorema da corona $\delta(D)$ é denso em $\left(\mathcal{M}\left(\mathcal{H}^{\infty}(D)\right), w^{*}\right)$; e portanto existe uma rede $\left(\delta_{\lambda_{\alpha}}\right)_{\alpha \in A}$, com $\left|\lambda_{\alpha}\right|<1$, convergindo na topologia $w^{*}$ para $n_{0}$.

Como $n_{0}, \delta_{\lambda_{\alpha}} \in \mathcal{M}\left(\mathcal{H}^{x}(D)\right), \forall \alpha \in A$ temos por (4.1) que

$$
\begin{gathered}
\left|\delta_{\lambda_{\alpha}}(f)\right|=\lim _{k \rightarrow \infty}\left|\widehat{f_{j_{k}}}\left(\phi_{T}\left(\delta_{\lambda_{\alpha}}\right)\right)\right|=1 \\
\mathrm{e} \\
\left|n_{0}(f)\right|=\lim _{k \rightarrow \infty}\left|\widehat{f_{j_{k}}}\left(\phi_{T}\left(n_{0}\right)\right)\right| \leq \beta<1 .
\end{gathered}
$$

Portanto $1=\lim _{\alpha}\left|\delta_{\lambda_{\alpha}}(f)\right|=\left|n_{0}(f)\right| \leq \beta<1$, o que nos leva a uma contradição.

Logo $\phi_{T}\left(\mathcal{M}\left(\mathcal{H}^{\infty}(D)\right)\right) \subseteq P\left(m_{0}\right)$, como queríamos.

Proposição 4.3.2 Seja $T: \mathcal{H}^{\infty}(D) \longrightarrow \mathcal{H}^{\infty}(D)$ um homomorfismo w-compacto. Então a aplicação $\phi_{T}:\left(\mathcal{M}\left(\mathcal{H}^{\infty}(D)\right), w^{*}\right) \longrightarrow\left(\phi_{T}\left(\mathcal{M}\left(\mathcal{H}^{\infty}(D)\right)\right),\|\cdot\|\right)$ é contínua.

Demonstração: Como T é w-compacto, segue do Teorema 1.2 .59 que $T^{*}: \mathcal{H}^{\infty}(D)^{\prime} \longrightarrow$ $\mathcal{H}^{\infty}(D)^{\prime}$ é $w$-compacto. Assim, como $\mathcal{M}\left(\mathcal{H}^{\infty}(D)\right) \subseteq B_{\mathcal{H}^{\infty}(D)^{\prime}}$, segue que $T^{*}\left(\mathcal{M}\left(\mathcal{H}^{\infty}(D)\right)\right)$ é relativamente $w$-compacto.

Agora, de $\phi_{T}=\left.T^{*}\right|_{\mathcal{M}\left(\mathcal{H}^{\infty}(D)\right)}$, temos que $\phi_{T}\left(\mathcal{M}\left(\mathcal{H}^{\infty}(D)\right)\right)=T^{*}\left(\mathcal{M}\left(\mathcal{H}^{\infty}(D)\right)\right)$ é relativamente $w$-compacto. Como $\phi_{T}\left(\mathcal{M}\left(\mathcal{H}^{\infty}(D)\right)\right)$ é $w$-fechado, segue que $\phi_{T}\left(\mathcal{M}\left(\mathcal{H}^{\infty}(D)\right)\right)$ é $w$-compacto.

Seja $L_{m_{0}}: D \longrightarrow P\left(m_{0}\right)$ a aplicação obtida no Teorema 4.1.5. 
Para cada $0<r<1$, considere o disco aberto $D_{r}(0)$. Como $L_{m_{0}}$ é um homeomorfismo sobre $\left(P\left(m_{0}\right), w\right)$ temos que $L_{m_{0}}\left(D_{r}(0)\right)$ é aberto em $\left(P\left(m_{0}\right), w\right)$.

Como $D=\bigcup_{n=2}^{\infty} D_{1-\frac{1}{n}}(0)$ e $\phi_{T}\left(\mathcal{M}\left(\mathcal{H}^{\infty}(D)\right)\right) \subseteq P\left(m_{0}\right)=L_{m_{0}}(D)$, temos que

$$
\phi_{T}\left(\mathcal{M}\left(\mathcal{H}^{\infty}(D)\right)\right) \subseteq L_{m_{0}}(D)=\bigcup_{n=2}^{\infty} L_{m_{0}}\left(D_{1-\frac{1}{n}}(0)\right) .
$$

Como para cada $n$, o conjunto $L_{m_{0}}\left(D_{1-\frac{1}{n}}(0)\right)$ é $w$-aberto e $\phi_{T}\left(\mathcal{M}\left(\mathcal{H}^{\infty}(D)\right)\right)$ é $w$ compacto, segue que existe $N_{0} \in \mathbb{N}$ tal que

$$
\phi_{T}\left(\mathcal{M}\left(\mathcal{H}^{\infty}(D)\right)\right) \subseteq L_{m_{0}}\left(D_{1-\frac{1}{N_{0}}}(0)\right) .
$$

$\operatorname{Assim} o_{T}\left(\mathcal{M}\left(\mathcal{H}^{\infty}(D)\right)\right) \subseteq L_{m_{0}}\left(D_{1-\frac{1}{N_{0}}}(0)\right) \subseteq L_{m_{0}}\left(\overline{D_{1-\frac{1}{N_{0}}}(0)}\right)$ e portanto

$$
\overline{\phi_{T}\left(\mathcal{M}\left(\mathcal{H}^{\infty}(D)\right)\right)}{ }^{\|\cdot\|} \subseteq L_{m_{0}}\left(\overline{D_{1-\frac{1}{N_{0}}}(0)}\right)
$$

ou seja. $\overline{\phi_{T}\left(\mathcal{M}\left(\mathcal{H}^{\infty}(D)\right)\right)^{\|\cdot\|}}$ é um fechado contido em um compacto, o que mostra que $\bar{\phi}_{T}\left(\mathcal{M}\left(\mathcal{H}^{\infty}(D)\right)\right) \|^{\|\cdot\|}$ é compacto.

Vamos agora provar que $\phi_{T}:\left(\mathcal{M}\left(\mathcal{H}^{\infty}(D)\right), w^{*}\right) \longrightarrow\left(\phi_{T}\left(\mathcal{M}\left(\mathcal{H}^{\infty}(D)\right)\right),\|\cdot\|\right)$ é contínua. Temos pelo Teorema 1.2 .39 que $\phi_{T}:\left(\mathcal{M}\left(\mathcal{H}^{\infty}(D)\right), w^{*}\right) \longrightarrow\left(\phi_{T}\left(\mathcal{M}\left(\mathcal{H}^{\infty}(D)\right)\right), w^{*}\right)$ é contínua: temos também que $I d:\left(\phi_{T}\left(\mathcal{M}\left(\mathcal{H}^{\infty}(D)\right)\right),\|\cdot\|\right) \longrightarrow\left(\phi_{T}\left(\mathcal{M}\left(\mathcal{H}^{\infty}(D)\right)\right), w^{*}\right)$ é um homeomorfismo, pois é uma aplicação contínua cujo domínio é um espaço compacto. Da composição entre $\phi_{T}:\left(\mathcal{M}\left(\mathcal{H}^{\infty}(D)\right), w^{*}\right) \longrightarrow\left(\phi_{T}\left(\mathcal{M}\left(\mathcal{H}^{\infty}(D)\right)\right), w^{*}\right)$ e $I d^{-1}$ : $\left(\phi_{T}\left(\mathcal{M}\left(\mathcal{H}^{\infty}(D)\right)\right), w^{*}\right) \longrightarrow\left(\phi_{T}\left(\mathcal{M}\left(\mathcal{H}^{\infty}(D)\right)\right),\|\cdot\|\right)$ segue que $\phi_{T}:\left(\mathcal{M}\left(\mathcal{H}^{\infty}(D)\right), w^{*}\right) \longrightarrow$ $\left(\phi_{T}\left(\mathcal{M}\left(\mathcal{H}^{\infty}(D)\right)\right),\|\cdot\|\right)$ é contínua, como queríamos.

Segue agora o principal teorema desta seção.

Teorema 4.3.3 Seja $T: \mathcal{H}^{\infty}(D) \longrightarrow \mathcal{H}^{\infty}(D)$ um homomorfismo. Então $T$ é compacto se, e somente se, $T$ é w-compacto.

Demonstração: Se $T$ é compacto, a Proposição 1.2 .57 garante que $T$ é $w$-compacto. Por outro lado, se $T$ é $w$-compacto, vamos provar que $T$ é compacto.

Tomemos uma sequência $\left(f_{j}\right)$ em $\mathcal{H}^{\infty}(D)$ com $\left\|f_{j}\right\| \leq 1$. Considere o conjunto $\mathcal{F}=$ $\left\{\widehat{T\left(f_{j}\right)}: j \in \mathbb{N}\right\} \subseteq \mathcal{C}\left(\mathcal{M}\left(\mathcal{H}^{\infty}(D)\right)\right)$. Vamos mostrar que $\mathcal{F}$ é limitado e equicontínuo e com 
auxílio do Teorema 1.2.52 (Arzela-Ascoli), provar que $\left(T\left(f_{j}\right)\right)$ possui uma subsequência convergente.

Como $\mathcal{H}^{\infty}(D)$ é uma álgebra uniforme, temos pelo Teorema 2.2.6(1) que $\left\|\widehat{T\left(f_{j}\right)}\right\|=$ $\left\|T\left(f_{j}\right)\right\|, \forall j \in \mathbb{N}$. Agora, como $\left\|f_{j}\right\| \leq 1$, a Proposição 4.2 .1 garante que $\left\|\widehat{T\left(f_{j}\right)}\right\|=$ $\left\|T\left(f_{j}\right)\right\| \leq 1, \forall j \in \mathbb{N}$, e consequentemente $\mathcal{F}$ é limitado.

Agora afirmamos que $\mathcal{F}$ é equicontínuo. De fato, a Proposição 4.3.2 garante que a aplicação $\phi_{T}:\left(\mathcal{M}\left(\mathcal{H}^{\infty}(D)\right), w^{*}\right) \longrightarrow\left(\phi_{T}\left(\mathcal{M}\left(\mathcal{H}^{\infty}(D)\right)\right),\|\cdot\|\right)$ é contínua. Logo, se $n_{0} \in$ $\mathcal{M}\left(\mathcal{H}^{\infty}(D)\right)$ e $\epsilon>0$, existem $\delta>0$ e $g_{1}, g_{2}, \cdots, g_{k} \in \mathcal{H}^{\infty}(D)$ tais que $\left\|\phi_{T}(n)-\phi_{T}\left(n_{0}\right)\right\|<\epsilon$, para todo $n \in \mathcal{M}\left(\mathcal{H}^{\infty}(D)\right)$ tal que $\sup _{i=1,2, \cdots, k}\left|n\left(g_{i}\right)-n_{0}\left(g_{i}\right)\right|<\delta$.

Portanto

$$
\begin{gathered}
\left|\widehat{T\left(f_{j}\right)}(n)-\widehat{T\left(f_{j}\right)}\left(n_{0}\right)\right|=\left|n\left(T\left(f_{j}\right)\right)-n_{0}\left(T\left(f_{j}\right)\right)\right|= \\
\left|\phi_{T}(n)\left(f_{j}\right)-\phi_{T}\left(n_{0}\right)\left(f_{j}\right)\right| \leq\left\|\phi_{T}(n)-\phi_{T}\left(n_{0}\right)\right\| \leq \epsilon, \forall n \in V\left(n_{0}, g_{1}, \cdots, g_{k}, \delta\right) .
\end{gathered}
$$

Logo, de acordo com a Definiçào 1.2 .51 , temos que $\mathcal{F}$ é equicontínuo.

Agora, pelo Teorema de Arzela-Ascoli, 1.2.52, $\mathcal{F}$ é totalmente limitado. Como $\mathcal{C}\left(\mathcal{M}\left(\mathcal{H}^{\infty}(D)\right)\right)$ é completo, segue do Teorema 1.2 .53 que $\mathcal{F}=\left\{\widehat{T\left(f_{j}\right)}: j \in \mathbb{N}\right\}$ é relativamente compacto.

Portanto existe uma subsequência $\left(\widehat{T\left(f_{j_{k}}\right)}\right)$ de $\left(\widehat{T\left(f_{j}\right)}\right)$ que converge. Agora, como $\mathcal{H}^{\infty}(D)$ é uma álgebra uniforme, temos pelo Teorema 2.2.6(1) que a transformação de Gelfand é um isomorfismo isométrico de $\mathcal{H}^{\infty}(D)$ sobre $\mathcal{H}^{\infty}(D)$. Logo $\left(T\left(f_{j_{k}}\right)\right)$ é convergente, e consequentemente $T$ é compacto, como queríamos demonstrar.

Observação 4.3.4 O resultado provado acima pode ser generalizado. Na verdade, se $T: \mathcal{H}^{\infty}(D) \longrightarrow B$ é um homomorfismo, onde $B$ é uma álgebra uniforme, então $T$ é compacto se, e somente se, $T$ é w-compacto. Este resultado foi demonstrado por P. Galindo e M. Lindström em [10].

Para demonstrar o próximo corolário, vamos precisar da seguinte definição.

Definição 4.3.5 Um espaço de Banach $X$ é um espaço de Grothendieck se todas as sequências $w^{*}$-convergentes em $X^{\prime}$ forem $w$-convergentes. 
Em [3], J. Bourgain provou que $\mathcal{H}^{\infty}(D)$ é um espaço de Grothendieck.

Corolário 4.3.6 Seja $T: \mathcal{H}^{\infty}(D) \longrightarrow \mathcal{H}^{\infty}(D)$ um homomorfismo. Se a imagem de $T$ está contida em $\mathcal{A}(D)$, temos que $T$ é compacto.

Demonstração: Seja $T: \mathcal{H}^{\infty}(D) \longrightarrow \mathcal{H}^{\infty}(D)$ tal que $T\left(\mathcal{H}^{\infty}(D)\right) \subseteq \mathcal{A}(D)$. Vamos mostrar que $T^{*}: \mathcal{A}(D)^{\prime} \longrightarrow \mathcal{H}^{\infty}(D)^{\prime}$ é $w$-compacto e consequentemente, pelo Teorema 1.2 .59 , temos que $T$ é $w$-compacto. Daí, pelo Teorema 4.3.3, concluímos que $T$ é compacto.

Seja $\left(\phi_{j}\right) \subseteq \mathcal{A}(D)^{\prime}$, com $\left\|\phi_{j}\right\| \leq 1$. Como a Proposição 1.2 .43 garante que $\mathcal{A}(D)$ é separável, temos pelo Teorema 1.2.45 que $B_{\mathcal{A}(D)^{\prime}}$ é $w^{*}$-metrizável. Como $B_{\mathcal{A}(D)^{\prime}}$ é $w^{*}$ compacto, existe uma subsequência $\left(\phi_{j_{k}}\right)$ de $\left(\phi_{j}\right)$ tal que $\phi_{j_{k}}$ é $w^{*}$-convergente. Agora, pelo Teorema 1.2.39. $T^{*}$ é $w^{*}$-contínua. Logo $T^{*}\left(\phi_{j_{k}}\right)$ é $w^{*}$-convergente em $\mathcal{H}^{\infty}(D)^{\prime}$. Como $\mathcal{H}^{\infty}(D)$ é um espaço de Grothendieck, segue que $T^{*}\left(\phi_{j_{k}}\right)$ é $w$-convergente. Pelo Teorema de Eberlein-Šmulian, 1.2.40, temos que $T^{*}$ é $w$-compacto, como queríamos.

Vamos dar um exemplo de um homomorfismo $w$-compacto de $\mathcal{H}^{\infty}(D)$ cuja imagem não está contida em $\mathcal{A}(D)$.

Exemplo 4.3.7 Sejam $0<r<1$ e $\varphi(z)=r z+(1-r)$. Se $|z|<1$, temos que

$$
|\varphi(z)|=|r z+(1-r)|<1 .
$$

Portanto $\varphi(D) \subseteq D$. Mais ainda, $\varphi(D) \varsubsetneqq D$. De fato, se $z=-\frac{1}{r}$, temos que $z \notin D$, mas $\varphi(z)=-r \in D$. Portanto segue da Propoisção 4.2 .4 que $C_{\varphi}: \mathcal{H}^{\infty}(D) \longrightarrow \mathcal{H}^{\infty}(D)$ é $w$-compacto.

$$
\text { Agora seja } f(z)=\left\{\begin{array}{l}
z,|z|<1 \\
2,|z|=1
\end{array} .\right.
$$

É claro $\left.f\right|_{D} \in \mathcal{H}^{\infty}(D)$. Agora, $C_{\varphi}(f)=\left.f \circ \varphi\right|_{D} \in \mathcal{H}^{\infty}(D)$ mas $f \circ \varphi \notin \mathcal{A}(D)$ pois $f$ não é contínua em $z=1$ e $\varphi(1)=1$.

Seja $l^{\infty}=\left\{\left(x_{j}\right)_{j \in \mathbb{N}}: x_{j} \in \mathbb{K}, \forall j \in \mathbb{N}\right.$ e $\left.\sup _{j \in \mathbb{N}}\left|x_{j}\right|<\infty\right\}$

Sejam $B$ uma álgebra uniforme e $T: \mathcal{H}^{\infty}(D) \longrightarrow B$ um homomorfismo. Mostraremos no Corolário 4.3.9 que se $B$ não contém cópia isomorfa do $l^{\infty}$, entào $T$ é compacto. Para 
provar tal resultado, enunciaremos o teorema a seguir, sem demonstração. A mesma pode ser encontrada em [3].

Teorema 4.3.8 Sejam B uma álgebra uniforme e $T: \mathcal{H}^{\infty}(D) \longrightarrow B$ um homomorfismo. Se $T$ não é $w$-compacto, então $T$ é um isomorfismo quando restrito a um subespaço $Z$ de $\mathcal{H}^{\infty}(D) . Z$ isomorfo ao $l^{\infty}$.

A. Ülger demonstrou o seguinte corolário em [34].

Corolário 4.3.9 Seja B uma álgebra uniforme que não contém, como espaço de Banach, cópia isomorfa do $l^{\infty}$. Então todo homomorfismo $T: \mathcal{H}^{\infty}(D) \longrightarrow B$ é compacto.

Demonstração: Vamos supor por absurdo que $T$ não é compacto. Pela Observação 4.3.4, T não é $w$-compacto. Agora, pelo Teorema 4.3.8, $T$ é um isomorfismo quando restrito a um subespaço $Z$ de $\mathcal{H}^{\infty}(D), Z$ isomorfo ao $l^{\infty}$. Logo $T(Z)$ seria uma cópia isomorfa ao $l^{\infty}$ em $B$, o que por hipótese é um absurdo. Portanto $T$ é compacto. 


\section{Referências Bibliográficas}

[1] R. Aron. P. Galindo, M. Lindström, Compact homomorphisms between algebras of analytic functions, Studia Math. 123 (3) (1997), 235-247.

[2] H. S. Bear, A geometric characterization of Gleason parts. Proc. Amer. Math. Soc. 16 (1965), 407-412.

[3] J. Bourgain, $H^{\infty}$ is a Grothendieck space, Studia Math. 75 (1983), 193-216.

[4] L. Carleson, Interpolations by bounded analytic functions and the corona problem, Ann. of Math. 76 (1962), 547-559.

[5] J. B. Conway, A Course in Functional Analysis, Springer-Verlag. New York. 1985.

[6] J. B. Conway, Functions of One Complex Variable, Second Edition, Springer-Verlag. New York. 1978.

[7] C. Cowen, B. Maccluer, Composition Operators on Spaces of Analytic Functions, CRC Press. Boca Raton. 1995.

[8] J. Diestel, Sequences and Series in Banach Spaces, Springer-Verlag. New York. 1984.

[9] N. Dunford, J. Schwartz, Linear Operators, Part I, Interscience Publishers. New York. 1958 .

[10] P. Galindo, M. Lindström, Gleason parts and weakly compact homomorphisms between uniform Banach algebras, Mh. Math. 128 (1999), 89-97.

[11] P. Galindo, M. Lindström, R. Ryan, Weakly compact composition operators between algebras of bounded analytic functions, Proc. Amer. Math. Soc. 128 (1999), 149-155. 
[12] P. Galindo, M. L. Lourenço, L. A. Moraes, Compact and weakly compact homomorphisms on Fréchet algebras of holomorphic functions, a aparecer, RT-MAT, 9910, (1999).

[13] J. B. Garnett. Bounded Analytic Functions, Academic Press. New York. 1981.

[14] A. M. Gleason, Function algebra.s, Seminars on Analytic Functions, Vol. 2, Institute for Advanced Study, Princeton. N. J., 1957.

[15] K. Hoffman, Banach Spaces of Analytic Functions, Dover Publications. New York. 1962.

[16] K. Hoffman, Bounded analytic functions and Gleason parts. Ann. of Math., 86 (1967), 74-111.

[17] K. Hoffman, Analytic functions and logmodular Banach algebras. Acta. Math., 108 (1962), 271-317.

[18] H. Kamowitz, The spectra of a class of operators on the disc algebra, Indiana Univ. Math. J., 27 (197\$) 5\$1-610.

[19] H. Kamowitz, Compact operators of the form $u C_{\varphi}$, Pacif. J. Math., 80 (1) (1979), 205-211.

[20] A. Kerr-Lawson, A filter description of the homomorfisms of $\mathcal{H}^{\infty}(D)$, Canad. J. Math, 17 (1965), 734-757.

[21] B. O. Koopman, Hamiltonian systems and tranformations in Hilbert space, Proc. Nat. Acad. Sci. USA, 17 (1931), 315-318.

[22] E. Kreyszig, Introductory Functional Analysis with Applications, John Wiley \& Sons. New York. 1989.

[23] R. Larsen, Banach Algebras, Marcel Dekker, Inc. New York. 1973.

[24] E. L. Lima, Espaços Métricos, I.MPA. Rio de Janeiro. 1977.

[25] M. L. Lourenço, L. A. Moraes, O. W. Paques, Espectros de Álgebras de Aplicações Holomorfas, Mini-Curso. 45 Seminário Brasileiro de Análise. Florianópolis. 1997. 
[26] G. M. Leibowitz, Lectures on Complex Function Algebras, Scott, Foresman and Company. Glenview. 1970.

[27] I. J. Maddox, Elements of Functional Analysis, Second Edition, Cambridge University Press. Cambridge. 1988.

[28] E. Nordgren, Composition operators, Canad. J. Math. 20 (1968), 442-449.

[29] W. Rudin, Real and Complex Analysis, Second Edition, McGraw-Hill. New York. 1974.

[30] J. V. Ryff, Subordinate $H^{p}$ functions, Duke Math. J. 33 (1966), 347-354.

[31] J. H. Shapiro. Composition Operators and Classical Function Theory, SpringerVerlag, New York, 1993.

[32] I. J. Schark, Maximal ideals in an algebra of bounded analytic functions, J. Math. Mech., 10 (1961). T35-746.

[33] J. H. Schwartz, Composition Operators on $H^{p}$, Thesis, Univ. de Toledo, 1968.

[34] A. Ülger, Some results about the spectrum of commutative Banach algebras under the weak topology and applications, Mh. Math. 121 (1996), 353-379. 